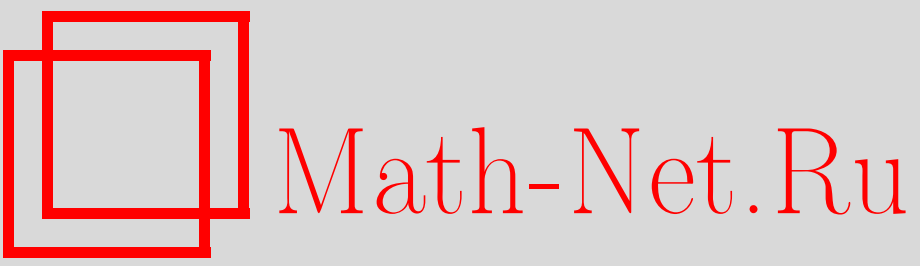

А. М. Вершик, Динамическая теория роста в группах: энтропия, границы, примеры, УМН, 2000, том 55, выпуск 4, 59-128

DOI: https://doi.org/10.4213/rm314

Использование Общероссийского математического портала Math-Net.Ru подразумевает, что вы прочитали и согласны с пользовательским соглашением

http://www . mathnet.ru/rus/agreement

Параметры загрузки:

IP: 54.196 .121 .252

26 апреля 2023 г., 15:26:07 


\title{
ДИНАМИЧЕСКАЯ ТЕОРИЯ РОСТА В ГРУППАХ: ЭНТРОПИЯ, ГРАНИЦЫ, ПРИМЕРЫ
}

\author{
А. М. ВЕРшИК
}

В статье идет речь:

1) о численных инвариантах счетных групп - энтропии, логарифмическом объеме, сносе, о фундаментальном неравенстве, связывающем их, и основанном на нем сравнении систем образующих групп; о задании групп с помощью метода МонтеКарло;

2) об эргодическом методе построения и изучения границ случайных блужданий, энтропии полиморфизма границы и связи с фундаментальным неравенством;

3) о геометрической реализации свободных разрешимых групп, их границах и 0 геометрическом подходе к построению нормальных форм в группах;

4) о локальных, локально свободных группах и о вычислении констант для них;

5) об энтропии в теории меры и в теории динамических систем и о вводимых вновь понятиях энтропии убывающей последовательности измеримых разбиений и вторичной энтропии колмогоровских автоморфизмов.

Библиограффия: 108 названий.

\section{СОДЕРЖАНИЕ}

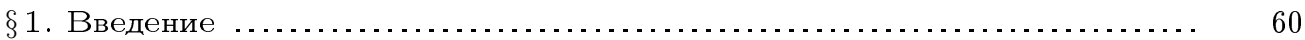

$\S 2$. Основные понятия. Фундаментальное неравенство . ............... 69

$\S 3$. Структура границ и связь фундаментального неравенства с полиморфизмом границы .................................... 77

$\S 4$. Свободные разрешимые группы .............................. 101

$\S 5$. Локальные и локально свободные группы ........................ 109

Добавление. О различных понятиях энтропии; энтропия убывающих последовательностей, вторичная энтропия .................... 113

$\S 6$. Энтропия дискретных фильтраций .............................. 115

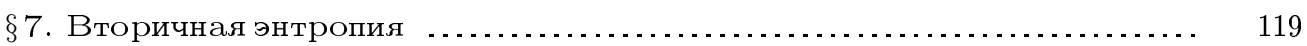

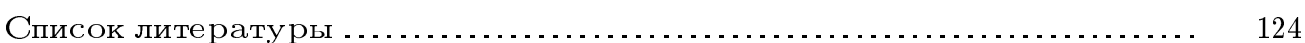

Работа выполнена при частичной поддержке Российского фонда фундаментальных исследований (грант № 99-01-00098). 


\section{$\S$ 1. Введение}

В этом обзоре рассматриваются асимптотические характеристики счетных групп, связанные с динамикой роста, случайными блужданиями, границами; изучаются новые классы примеров групп с этой точки зрения. Мы приводим также ряд известных и новых результатов о границах групп, о численных характеристиках групп и обсуждаем общие вопросы энтропийной теории.

Основной материал настоящей статьи связан с энтропией в группах, точнее, с энтропией случайных блужданий на группах, и ее применением к случайным блужданиям, гармоническому анализу на группах, но в первую очередь к теории асимптотических характеристик груп, сравнению систем образующих и к возможности равномерного распределения на экспоненциальных группах. Энтропия случайных блужданий на группах была введена Авецом в 1972 г. [1] и переоткрыта мной несколькими годами позже. Но в специальном случае направленного блуждания на градуированном графе энтропия блуждания фактически рассматривалась еще в 1962 г. Гарсиа [2], а в несколько иной форме близкое понятие энтропии в связи с теорией границ рассматривалось в 1964 г. Г. Фюрстенбергом [3]-[5]. Систематический и подробньй анализ энтропии случайных блужданий, их границ и связанных с ними проблем был начат автором в работах с В. Каймановичем [6]-[8] и продолжен в работах Ф. Ледраппье, В. Каймановича, И. Деренника и др., последовавших за ними. К настоящему времени этой теме посвящено много статей, имеются систематические обзоры (например, [9]-[18]) первого этапа энтропийной теории случайных блужданий. Поэтому мы не ставили целью давать здесь обзор и даже приводить сколько-нибудь полньй перечень литературы об энтропии, границах и свойствах случайных блужданий; цели данной статьи несколько иные. В частности, они состоят в применении энтропии для изучения асимптотик в экспоненииальных әруппах, в обсуждении новых подходов и их иллюстрации для описания гранищ на примерах некоторых групп. В последние годы наблюдается заметное повьшение интереса со стороны физиков к разнообразным задачам о стохастических дискретных моделях и связанных с ними асимптотиками. Случайные блуждания на группах дают богатьй материал для таких моделей, и привлечение внимания к таким связям есть одна из целей обзора. Столь же активно идет работа по вычислению и определению границ групп и однородных пространств. В этой работе мы также затрагиваем вопрос о вычислении границ с новой точки зрения.

Новые результаты статьи, помимо установления фундаментального неравенства в должной общности и вьвода следствий из него, состоят в придании ему нового смысла с помошью марковской динамики на гранище ( $(2$ и 3$)$, в абстрактном описании метода нахож дения границ Пуассона-Фюрстенберга и его применения к свободным разрешимьм группам ступени два и для некоторых сплетений и в конструкции новой (топологической) модели свободных разрешимых групп (§4). Мы также продолжаем обсуждение нового класса групп - локальных и локально свободных групп (§ 5$)$, и воспроизводим, возможно, первьй нетривиальньй пример использования фундаментального неравенства с оценками введенных констант, полученных в недавней работе [19], для локально свободных групп. Мы объясняем причину того, почему имеет место строгое неравенство в фундаментальном неравенстве для локально свободной групшы и группы кос. Интересен также обнаруженньй при этом эффект стабилизации констант при стремлении числа образующих к бесконечности. 
Наконец, в добавлении к статье ( $(6$ и 7) приводятся два тесно связанных между собой новых понятия энтропии - энтропии убывающих последовательностей и вторичной энтропии колмогоровских автоморфизмов, и ставятся задачи, связанные с этими понятиями.

Прежде чем переходить к более подробному описанию проблем и результатов, отметим, что вся современная энтропийная проблематика так или иначе связана с колмогоровской энтропией динамических систем и, если двигаться назад по времени, с теорией информации Шеннона. Но, быть может, самьй глубокий вклад в энтропийную технику, складывавшуюся уже после открытия А. Н. Колмогорова, был сделан В.А. Рохлиным, а также Я.Г. Синаем. Техника измеримых разбиений, разработанная в 40-х гг. В. А. Рохлиным, была тем языком, на котором А. Н. Колмогоров изложил свое определение энтропии динамической системы. Одна из целей этой статьи показать, что несмотря на внешнее несходство ситуаций, в которых рассматривается энтропия в этой статье (теория групповых характеристик и случайных блужданий на группах) и энтропия в теории преобразований с инвариантной мерой, - способ образования понятий и техника, разработанная в те годы, с успехом применима и в этой ситуации. По поводу своего определения Авец [1] замечает, что он следует примеру колмогоровской энтропии динамической системы, но эти два понятия, конечно, очень различны - сходство остается чисто внешним до тех пор, пока не вскрьваются более глубокие связи с границами, о некоторых из них и идет речь в статье.

Мотивировка, которая привела автора к понятию энтропии групп с мерой, состояла в следуюшем. Если в конечной групше выбрана произвольная система образующих и мера (например, равномерная) с носителем на этой системе, то ее свертки стремятся к равномерной мере - мере Хаара; имеется обширная литература, посвященная оценкам скорости этой сходимости и приложениям этих результатов. Если группа имеет полиномиальный или даже субэкспоненциальный рост, то также можно говорить об асимптотической равнораспределенности сверток меры на группе или на $G$-пространстве, что столь же активно обсуждалось во многих работах. Но если группа экспоненциальна, то, вообще говоря, никакой равномерной распределенности сверток нет, и встает вопрос о возможности выбора такой системы образующих и меры на них, свертки которых имеют распределение, наиболее близкое к равномерному, или даже асимптотически совпадают с равномерным. Такие меры могут служить средством генерации элементов самой группы. Этот вопрос имеет практическое значение: группу можно задавать не только с помощью образующих и соотношений, что всегда связано с проблемой тождества слов и другими трудностями логического характера, и не только с помошью представления групшы, которое определяет лишш действие образующих и оставляет другие элементы заданными лишш неявно, - но и с помощью метода Монте-Карло, т.е. задавая меру на образующих и обратных к ним (например, равномерную) и генерируя элементы группы случайным образом, составляя слова из последовательных независимых образуюших (и обратных к ним). Это есть то, что назьвают случайньм блужданием на группе. Спрашивается, получим ли мы асимптотически полное представление об элементах групшы в результате такого процесса. Более точно, какова доля среди всех элементов группы данной длины тех из них, которые мы получим в результате нашего процесса с вероятностью близкой к единице; будет ли эта доля асимптотически исчерпьвать все элементы группы или такой выбор даст лишь экспоненциально малую часть группы? В этом и состоит во- 
прос о равномерной распределенности, о котором шла речь вьше. По существу мы обсуждаем проблему связи между асимптотикой равномерных мер на словах данной длины и асимптотикой случайных произведений образующих.

Конечно, ответ на поставленньй вопрос зависит от выбора образующих и мер на них, что отличает проблему для экспоненциальных групп от остальных групп. Отчасти ответ содержится в так называемом фундаментальном неравенстве, обсуждаемом ниже. Положительный ответ на этот вопрос дает оправдание случайного генерирования, отрищательный - в нашем случае означает, что метод Монте-Карло порождает асимптотически лишь экспоненииально малую часть әруппь.

Все это - тема второго параграфа. В нем даются необходимые определения и отмечается, что сверхпопулярньй в литературе рост числа слов (логарифмический объем) сам по себе не содержит глубокой информации об экспоненциальных группах. Гораздо более эффективно изучать его вместе с энтропией, которая в данном контексте показывает, какая информация об одной образующей содержится в среднем в типичном элементе групшы, записанном словом в этих образующих, или, по-другому, учитьвает, сколькими способами можно записьвать данньй элемент группы словами в данных образующих. Тем самым, энтропия измеряет неопределенность, содержашуюся в ансамбле всех слов данной длины, снабженных сверточной степенњю исходной меры. В экспоненциальных группах возникает еше одна важная характеристика - снос, т.е. линейная скорость роста слов. Именно эти три характеристики (рост, энтропия, снос) и связаны замечательньм фундаментальным неравенством:

$$
h \leqslant l \cdot v
$$

которое, несмотря на простоту доказательства, имеет очень важное и глубокое значение. В конце второго параграф̆а мы уточняем, как использовать его в качестве средства для сравнения асимптотик равномерной и сверточной мер. Если неравенство становится равенством, то, как отмечалось, метод случайного генерирования является адекватным, а в противном случае мы получаем лишш экспоненциально малую часть группы. Таким образом, появляется объективный способ сравнения систем образуюших: какая из них "в большей степени” порождает группу.

Само неравенство в частных формах появлялось и ранее. Уже в первой статье Авеца [1] было отмечено почти очевидное похожее неравенство $h \leqslant v$, а в еще более ранней работе Милнора [20] ставился вопрос о связи роста (того, что далее мы назьваем логарифмическим объемом) и характеристик случайного блуждания типа спектрального радиуса, которьй несколькими годами ранее изучал Кестен [21]. Неравенство отмечается также в статье В. Каймановича [22] для случая фундаментальной групшы многообразий отрицательной кривизны, а в его недавней работе [9] оно исследуется в случае блужданий на деревьях; этот класс пересекается с изучаемым здесь групповым случаем только по свободным группам. В нашей статье мы придаем фундаментальному неравенству общий характер в алгебраических (в первую очередь, групповых) ситуациях; его связь с хаусдорфовой размерностью гармонической меры на границе, фрактальностью и др. в общем случае еше предстоит понять. Задачи вычисления указанных характеристик (роста, энтропии, сноса) и фундаментального неравенства для разных групп, различных систем образующих и мер на них, соотнесение этих характеристик со свойствами гармонических мер на граничах и с 
предельными распределениями функиионалов на них представляются главными проблемами в этом круге вопросов. В настояшее время ответы известны лишш в единичных случаях; для различных дискретных подгрупп группы $S L(2, \mathbb{R})$ эти задачи частично рассматривались, они имеют прямое отношение к геометрии и к задачам, возникаюшим в статистической физике. Нечего и говорить, что для решеток в полупростых группах Ли вопросы чрезвычайно сложны и интересны.

Наиболее важная проблема - описать те сильно экспоненииальнье группь и системы образующих в них, для которых фундаментальное неравенство превращается в равенство, и описать әкстремальнье системы образующих в группах, где этого свойства нет. Последнее означает выбор той системы образующих и меры на ней, для которых неравенство ближе всего к равенству, точнее, для которых введенный во втором параграфе показатель $q$ принимает наибольшее значение. Вполне возможно, что экстремальная система образуюших или система, сколь угодно близкая к экстремальной, существует во всякой групп, но заведомо ясно, что если она и сушествует, то отличается от привычных систем образующих для некоторых групп. Например, для групп кос и локально свободных групп это следует из приводимых в статье результатов работы [19]. Пока известные примеры образуют сравнительно узкий класс груп - виртуально свободные групшы (т.е. группы со свободной подгруппой конечного индекса) и близкие к ним, - мы назьваем их свободноподобньми.

Попутно заметим, что можно ослаблять условие независимости образующих для генерирования элементов группы, например, запрещать ставить рядом образующую и обратную к ней, продуцируя, таким образом, процесс с марковскими, а не независимьми приращениями; для свободной группы это сразу дает однозначность представления элемента. Но в общем случае такая модификация бесполезна, так как уменьшение длин слов в несвободной группе происходит не столько за счет таких сокращений, сколько за счет соотношений между образуюшими, которые могут быть очень длинными (например, в разрешимых группах), поэтому введение ограничений на случайный процесс генерирования, учитываюших соотношения, слишком усложняет дело. Но более сложные процессы генерирования могут оказаться полезными в специальных случаях (см., например, доказательство эргодических теорем для свободных полугрупп в недавней работе [23]). С другой стороны, оправдание независимости процесса генерирования, т.е. классического случайного блуждания, лежит в его связях с гармоническим анализом на группах, с обширной теорией произведений случайных матрищ, с гармоническим анализом и со многими другими вопросами.

Таким образом, энтропийная тема оказьвается в существе дела в вопросах роста и распределений на группах. Но, оказывается, и в более техническом смысле динамический подход вскрывает более глубокую связь колмогоровской энтропии и теории границ Пуассона-Фюрстенберга для случайных блужданий. Это - предмет третьего параграфа.

В начале третьего параграфа мы излагаем в основном известные факты о границах групп - границе-выход (= метрическом абсолюте), стационарной границе, границе Пуассона-Фюрстенберга - все они в данном случае совпадают. Теория границ отвечает за самую глубокую и интересную часть теории асимптотического поведения траекторий марковского процесса и всего гармонического анализа на группах. Нахож дение границ Мартина и других границ для конкретных групп и пространств и их идентификация с другими геометрическими объектами - весьма интересная и слож- 
ная проблема, которой посвяшено много работ. Начало их в групповой ситуации положено работой Е. Б. Дьнкина и М. Б. Малютова [24], где выгислялась граница Мартина для свободной групшы с мерой на образуюших. С тех пор вычислено немало в том числе и групповых примеров, в которых общая ситуация сходна со свободной группой. Среди многих работ на эту тему мы упомянем здесь лишш недавние результаты Каймановича-Мазура о совпадении этой гранишы с границей Терстона [25], [26] для пространств Тейхмюллера и более раннюю работу К. Сериес [27] о границе Мартина фундаментальных групп поверхностей. По-видимому, главная трудность лежит в том, как выбрать термины для описания границ.

На наш взгляд наиболее убедительное описание гранищ должно использовать комбинаторные или геометрические термины. "Словарные" (в терминах слов в алфавите образующих) описания, как мы увидим, работают не всегда, а геометрические сильно зависят от происхождения групшы. Однако есть некоторая общая схема нахождения грании, которую мы излагаем в третьем параграфе и которая является вариачией “әргодического метода", широко применявшегося в ряде задач нахождения инвариантных мер, характеров, асимптотической теории представлений и т. д. Это - простое следствие обших теорем типа эргодической теоремы, утверждающее, что задача сводится к нахождению пределов последовательностей функций (мер) вполне определенного вида. Разумеется, вычисление этих пределов есть конкретная задача, условия которой определяются группой или графом, и проведение этих вычислений может оказаться весьма сложным.

Поскольку наш подход к теории границ и структур на ней базируется на идеях эргодической теории пространств Лебега, то мы рассматриваем бернуллиевскую модель границы, иначе говоря, сначала вводится пространство приращений процесса, которое в данном случае есть бесконечное прямое произведение множества образуюших, с продакт-мерой, сомножитель которой есть симметричная мера на образующих, а различные границы есть фактор-пространства по тому или иному измеримому разбиению, задаваемому как предел цилиндрических разбиений. В групповом случае все интересные метрические границы совпадают и граница нетривиальна (не сводится к одной точке), как било установлено в [7], [8] и независимо в [11], в том и только том случае, когда энтропия блуждания (әнтропия группь с мерой) положительна. Именно этот, на первый взгляд неожиданный, результат показал пользу энтропии случайных блужданий. Новое объяснение этого факта, связанное с полной положительностью энтропии автоморфизма Бернулли, дано ниже.

Затем мы делаем следующий шаг и отвечаем на вопрос: что означает фундаментальное неравенство в предельной форме, т.е. как изучаемые характеристики интерпретируются в терминах границ случайного блуждания. Мы привлекаем еще одно, пока не слишком распространенное, понятие - полиморфизм, и даем новую трактовку фундаментального неравенства, используя многозначное преобразование гранищы блужданий, названное полиморфизмом гранииы.

Объясним это обстоятельство подробнее. Граница группы с мерой определяется как фактор-пространство пространства траекторий блуждания по разбиению на классы с одинаковым асимптотическим поведением (граница-выход). В рассматриваемых нами терминах это можно высказать так - граница группы с мерой есть фактор-пространство бесконечных слов в алфавите из образующих по измеримому замьканию следующего отношения эквивалентности: два бесконечных слова эквива- 
лентны, если они как последовательности совпадают, начиная с некоторого места, а произведения их начал - равны как элементы группы. Это разбиение неинвариантно относительно левого сдвига, поэтому на фактор-пространстве сдвиг не индуцирует однозначного преобразования. Однако сдвиг индуцирует многозначное преобразование - полиморфизм, играющее ключевую роль. Слово и понятие "полиморфизм", равнозначные понятию "марковского отображения пространств Лебега с инвариантной мерой”, были введены в [28] для того, чтобы подчеркнуть намерение изучать этот объект с точки зрения эргодической и энтропийной теории. Мы приводим несколько определений, связанных с понятием полиморфизма, и, в частности, вводим понятие энтропии, обобщающее энтропию автоморфизма.

Тождественность энтропии вводимого здесь канонического полиморфизма границы и энтропии группы с мерой показывает, что фундаментальное неравенство есть обобщение неравенства между топологической и метрической энтропиями для этого полиморфизма. Энтропия группы выступает в роли энтропии канонического полиморфизма, а произведение логарифмического объема на снос - в роли его топологической энтропии в надлежащей (уточненной) топологии. Таким образом, энтропия группы действительно есть колмогоровская энтропия марковского процесса (полиморфизма) на гранище. Эта точка зрения объясняет и энтропийньй критерий тривиальности границы - он просто является обобшением так назьваемой полной положительности энтропии бернуллиевского сдвига, состоящей в том, что у автоморфизма Бернулли нет нетривиальных факторов с нулевой энтропией. Как известно, из теорем Пинскера и Синая-Рохлина следует, что тем же свойством обладают $K$-автоморфизмы и только они. Наш полиморфизм задает случайное блуждание по траекториям канонического действия исходной групшы на гранище - в чем характеристическое свойство этого “вторичного” случайного блуждания и каковы его свойства в конкретных примерах, по-видимому, интересная проблема.

С другой стороны, эта интерпретация ставит новые вопросы: если фундаментальное неравенство - строгое, какие меры на границе имеют максимальную энтропию и в чем их групповой смысл? Иначе говоря, если гармоническая мера не есть мера с максимальной энтропией, то как получить последнюю с помощью групповых соображений? Объяснение строгости неравенства кроется в весьма нетривиальном обстоятельстве, которое иллюстрируется на примере локально свободной группы (эффект “запирания", см. §5). Оно же подсказьвает новый неожиданный способ обобшения случайных блужданий, который и должен привести к мере с максимальной энтропией: обобщенное блуждание должно состоять в том, что образующие добавляются не только в конец, слова, но и в середину по определенному правилу. Мы оставляем до другого раза более подробный анализ этой возможности. Напомним лишш, что до сих пор меры с максимальной энтропией воспринимались лишш как гиббсовские меры и не имели интерпретации, связанной напрямую с блужданиями.

Важньй вопрос о явном описании можно связать с нормальными формами элементов группы. Нормальной формой в әруппах с заданной системой образующих называется отображение, сопоставляющее каждому элементу групшы некоторое слово (нормальную форму), представляющее этот элемент, иначе говоря, нормальная форма есть сечение в расслоении свободной группы над данной группой. Границу, 
определяемую как фактор-пространство пространства всех бесконечных слов в алфавите образуюших, иногда можно описать и как его подпространство, т.е. как некоторое множество бесконечных неприводимых слов, или, если угодно, как множество нормальных форм бесконечных слов. Например, для свободной группы дело обстоит именно так: гранища есть пространство всех несократимых слов (теорема Дьпкина-Малютова [24]). Точно так же обстоит дело с группами поверхностей, группами с марковской нормальной формой и др. Мы доказываем более общую теорему, состоящую в том, что всякая стабильная нормальная форма определяет изоморфизм между некоторым пространством бесконечных слов и границей.

Но чаще всего, как видно из дальнейшего, стабильную нормальную форму не удается найти (может быть, ее нет). Например, найти минимальную нормальную форму означает решить сложную комбинаторную изопериметрическую задачу, и проверить ее стабильность тем более трудно; нестабильность нормальной формы означает, что она плохо согласована с групповой операцией. Так или иначе встает вопрос об иных способах описания гранииы групn с мерой. Гранишы групп и, следовательно, гармонические функции на группах совсем не обязательно связаны со стабильной нормальной формой. Примеры этому - решаемая в $§ 4$ задача об описании гранищ свободных разрешимых групп ступени два и сплетений. Однако сам метод доказательства полноты границы остается тем же, что и случае стабильной нормальной формы. Этому посвящен конец третьего параграфа - приведенная теорема, по-видимому, аккумулирует все, что можно сказать общего о методе отыскания границы, и обобщает в духе эргодического метода почти все имеюшиеся описания. Конкретные вычисления могут быть сложными, они зависят от конкретных особенностей групп.

В четвертом и пятом параграфах мы переходим к конкретным классам групп и примерам вычислений. Мы выделяем два класса групп - первый класс групп изучался с давних пор в алгебре, но не привлекался к задачам подобного типа - это свободные разрешимые группы. Ранее [8], [29] исследовались случайные блуждания на похожих группах - сплетениях решеток с циклическими группами; эти группы служили хорошим материалом для различных примеров и контрпримеров; сплетения также являются разрешимыми группами ступени 2 с бесконечнопорожденным коммутантом. Эти примеры сплетений с произвольной финитной мерой, имеющие нетривиальную границу, широко использовались в теории факторов [30], [31]. Но сейчас стало ясно, что свободные разрешимые групшы доставляют гораздо более естественные примеры такого рода и более приспособлены для задач такого рода. Mb даем новую топологическую модель свободной разрешимой группь ступени два с произвольным числом образующих и полностью описываем ее границу. Описание границы-геометрическое, а не "словарное", т.е. оно дано не в терминах нормальных форм, а в геометрических терминах, подобно абсолюту плоскости Лобачевского. С помошью того же способа мы даем описание границы сплетений решеток с циклической группой, что оставалось до последнего времени открытым вопросом.

Одно общее замечание сводит изучение траекторий блуждания на конечнопорожденной группе (т.е. блуждания на графе Кэли) к изучению классов эквивалентных траекторий простого случайного блуждания на обычной решетке ранга, равного числу образуюших групшы; понятие эквивалентности траекторий определяется группой. Хотя эта эквивалентность в общем случае является тавтологической переформулировкой эквивалентности слов, в примерах со свободной разрешимой группой эта эк- 
вивалентность описывается очень просто, и задачи о блужданиях на группе сводятся к изучению функционалов геометрической природы от путей простых блужданий на решетке. Возможности этого приема далеки от исчерпания и полезны не только для изучения групп, но, как мы думаем, и в обратном направлении - придании алгебраического характера некоторым моделям статистической физики. Этот прием сводит вычисление грании и другие асимптотические вопросы о блужданиях на группах $\kappa$ изучению $G$-инвариантных функционалов от траекторий классических простых блужданий на решетках.

Второй класс групп является относительно новым, он введен несколько лет тому назад автором [32], [33] под влиянием тематики, связанной с уравнением Янга-Бакстера и теорией групп Кокстера, - это так называемые локальныегруппы и, в частности, локально свободные групшы, которые более подробно рассмотрены в [34], [35]. В локальной группе образующие линейно упорядочены, они коммутируют, если они не стоят рядом, а соседние подчинены некоторым коммутационным соотношениям. В локально свободных группах, кроме того, пары соседних образующих порождают свободную группу. Таким образом, локально свободные группы являются свободными группами в многообразии локальных групп, и потому группы кос, например, являются их фактор-группами. Но локально свободная группа вкладьвается в группу кос как подгруппа, порожденная квадратами образующих. Поэтому мы получаем возможность давать двусторонние оценки некоторых констант для группы кос, если они известны для локально свободной группы. В работе [19] для локально свободной группы найдены оценки констант, описьваемых в данной работе, - роста, сноса и энтропии. Оказалось, что фундаментальное неравенство является строгим: естественная система образуюших не дает равенства, и неизвестно, сушествует ли в этой группе другая система образующих, дающая равенство. Отсюда можно вьвести, что для классических образуюших групш кос равенства также не будет. Упоминавшееся выше объяснение строгости неравенства и идея о том, как получить меру с максимальной энтропией, иллюстрируются на примере локально свободной групшы, такая же интерпретация может быть дана для групшы кос.

Для комбинаторного изучения локально свободной группы использована геометрическая техника, связанная с понятием груды (heap), которое возникло по другому поводу в связи с работой [36] и рассматривалось подробно в [37], [38]. Мы даем описание границы этой группы с помощью нормальной формы. Но наиболее неожиданный эффект в том, что при стремлении числа образующих $к$ бесконечности все три константы стабилизируются $к$ некоторым конечным значениям. Например, рост (логарифмический объем) стремится с увеличением числа образуюших к числу, равному логарифмическому объему свободной групш с четырьмя образуюшими. Более подробные результаты о локально свободной группе см. в [19].

Общий вывод из анализа рассматриваемых примеров в этих двух параграфах состоит в том, что естественные алгебраические примеры, возникающие из анализа блужданий на әруппах, приводят к моделям, имеющим физическую подоплеку. Для локально свободных групп - это модели направленного роста, для свободных разрешимых групп мы приходим к вопросам, близким к проблемам просачивания. При этом кажушаяся на первый взгляд громоздкость группы (например, свободной разре- 
шимой группы) оказывается в конце концов полезной, если найти наглядную геометрическую реализацию ее элементов (циклов в случае свободной разрешимой группы, груды в случае локально свободной группы).

В добавлении к статье, после нескольких замечаний об истории энтропий, мы приводим два новых определения, связанных с основным текстом общей энтропийной темой. Первое- определение общей тополого-метрической энтропии убъвающих последовательностей разбиений. Это определение включает нормировку (скейлинг), которая однозначно определяется типом самой последовательности. Для экспоненциальной нормировки эта энтропия с иным, более частньм, определением вводилась и использовалась в самом начале возникновения теории убывающих последовательностей (см. обзор [39]). Оказалось, что предлагаемьй здесь скейлинг дает серию инвариантов произвольных (не обязательно диадических) последовательностей измеримых разбиений. Другое понятие - вторичной энтропии регулярных процессов $u K$-автоморфизмов - возникло по аналогии с предыдущим и также имеет тополого-метрический характер, но связано совсем с другой темой-небернуллиевскими колмогоровскими системами. Их открыл в 70-х гг. Д. Орнштейн, а несчетное множество попарно неизоморфных небернуллиевских $K$-автоморфизмов было описано впервые в [40]; до этого было популярно мнение, что всякий автоморфизм с вполне положительной энтропией изоморфен бернуллиевскому. Бернуллиевские эндоморфизмы и условие VWB Орнштейна аналогичны соответственно стандартньм убывающим последовательностям и критерию стандартности [39]. Эта аналогия и стала источником определения вторичной энтропии и параллельного изучения обоих объектов. В дальнейшем появилось много примеров небернуллиевских $K$-автоморфизмов, среди которых наиболее плодотворньй пример рассмотрен С. Каликовым - случайное блуждание по траекториям автоморфизма Бернулли; однако практически никаких конструктивных инвариантов построено не было. Точно так же как введенные в шестом параграфе понятия энтропий дают систему инвариантов убываюших последовательностей измеримых разбиений, вторичная энтропия, определяемая в седьмом параграфе, - кандидат на такую систему инвариантов для эндоморфизмов; она измеряет $\varepsilon$-энтропию множества условных мер на будушем при фиксированном прошлом. Любопытно отметить, что $\varepsilon$-энтропия в пространстве мер измеряется относительно метрики Канторовича-Рубинштейна, с завидным постоянством появляюшейся во всех подобных задачах. Оба вводимых инварианта связаны как с динамической теорией энтропии, так и с теорией $\varepsilon$-энтропии: несколько упрощая ситуацию, можно сказать, что рассматривается асимптотическая $\varepsilon$-энтропия последовательности компактов, строяшихся с помошью нормировки (скейлинга) по последовательности разбиений в первом случае или фрагментов случайного процесса - во втором.

В недавнем примере П. Шилдса и К. Мартон [41] построен пример $K$-автоморфизма, у которого в наших терминах вторичная энтропия имеет скейлинг выше линейного, в противоположность $\sqrt{n}$ в примере Каликова. Описание возможных скейлингов, появляющихся в определении вторичной энтропии, - одна из наиболее интересных проблем в теории колмогоровских автоморфизмов.

В процессе работы над статьей автор беседовал со многими коллегами и учениками и выражает им благодарность за дискуссии и ссылки на литературу. Среди них М. Концевич, В. Кайманович, Ю. Матиясевич, Т. Нагнибеда, С. Нечаев, А. Шмелькин, J.-P. Thouvenot, X. Viennot, P. Shields, P. Dehornya, Y. Guivarch, D. Epstein, 
C. Series, R. Kenyon, студенты А. Горбульский, С. Добрынин, А. Дюбина. Автор благодарит также профессора A. Comtet и лабораторию LPTMS университета Париж-11 и Институт высших научных исследований (IHES), где завершалась работа над статьей, за гостеприимство. Первая часть работы рассказьвалась на конференции, посвященной 80-летию В.А. Рохлина, в августе 1999 г. в институте Эйлера в С.-Петербурге, на конференции в Уорике (март 2000 г.) и на семинарах в университете Париж-6, а содержание $\S 3$ и 4 - на семинарах ПОМИ, MИАН и IHES, краткая публикация этой части содержится в сборнике [42]. Настоящей статьей мы хотели бы привлечь внимание к новым аспектам теории случайных блужданий и асимптотикам на экспоненциальных группах, новым классам примеров групп и энтропийной теории. Доказательства некоторых из основных утверждений лишь намечены, более подробное изложение и развитие этих тем будет предметом других публикаций.

\section{§ 2. Основные понятия. Фундаментальное неравенство}

Пусть $G$ - счетная конечнопорожденная группа, $S=\left(g_{1}, \ldots, g_{k}, g_{1}^{-1}, \ldots, g_{k}^{-1}\right)$ симметричное множество образуюших группы $G$ и обратных к ним (т.е. образующих $G$ как полугруппы), а $\mu$ - симметричная (т.е. $\mu\left\{g_{i}\right\}=\mu\left\{g_{i}^{-1}\right\}>0$ ) вероятностная мера с носителем $S$, т.е. $\mu(S)=1$.

Определим три константы, связанные с тройкой $(G, S, \mu)$.

2.1. Логарифмический объем. Первая константа определяется только системой образуюших и хорошо известна. Под длиной элемента $g$ группы $G$ относительно данной системы образуюших $S$ мы понимаем минимальное число $l(g)$ символов в записи элемента $g$ как слова в алфавите $S$. Функция $\rho(g, h)=l\left(g h^{-1}\right)$ определяет правоинвариантную "словарную" метрику на группе (см. [43]). Пусть $W_{n}$ - множество слов в группе $G$ длины $n$ относительно образуюших $S$ (т.е. сфера радиуса $n$ с центром в единице в словарной метрике), а $|\cdot|$ - число элементов в множестве.

ОПРЕДЕЛЕНИЕ 1. Логарифмическим оббемом группы $G$ в заданной системе образуюших $S$ назовем число

$$
v_{S}=\lim _{n \rightarrow \infty} \frac{\ln \left|W_{n}\right|}{n} .
$$

Эта константа измеряет экспоненциальную скорость роста числа слов. Она изучалась во многих работах. Группы, для которых существует система образуюших $S$ с $v_{S}>0$, называются экспоненциальныцми. В этом случае для любой системы образуюших логарифмический объем больше нуля.

Вот некоторые из важнейших свойств этой характеристики: если логарифмический объем равен нулю (т.е. группа имеет субэкспоненциальньй рост), то группа аменабельна (см. [44]); однако среди аменабельных групп есть, конечно, и экспоненциальные - например, свободные разрешимые группы, о которых пойдет речь далее; полиномиальньй рост имеют только абелевы, нильпотентные и их конечные расширения [45], сушествуют группы с промежуточным между полиномиальным и экспоненциальным ростом [46] и т. д. Сам по себе логарифмический объем зависит от выбора образующих и в очень слабой мере отражает свойства группы. Очень многие группы, важные в топологии, алгебре, теории динамических систем и т.д., - экспоненциальны, например, таковы фундаментальные группы поверхностей отрицательной кривизны, решетки в полупростых группах и др. Много работ посвящено изучению производящей 
функции числа слов и выяснению ее рациональности (см. монографиию [47], [48]-[50] и литературу там).

2.2. Мера и соответствующее случайное блуждание на группе. Пусть теперь $\mu$ - симметричная вероятностная мера на группе $G$ с конечным носителем $S$, и пусть $\mu^{* n}-$ ее $n$-я свертка. То есть

$$
\mu^{* n}(A)=(\underbrace{\mu \times \cdots \times \mu}_{n})\left\{\left(g_{1}, \ldots, g_{n}\right): g_{1} \cdots g_{n} \in A\right\}
$$

Если $\mu$ - равномерная мера на $S$ (на образующих и их обратных), то ее значение $\mu^{* n}(g)$ на элементе $g$ пропорционально числу способов представления данного элемента в виде слова длины $n$ в алфавите $S$, таким образом, свертки учитывают кратности представления элементов группы, т.е. соотношения между образуюшими.

Пара $(G, \mu)$ задает случайное блуждание на группе, т.е. марковский процесс с переходной вероятностью

$$
P(w g \mid w)=\mu(g)
$$

(правое блуждание на групп) и начальным состоянием (в нулевой момент времени) в единице групшы. Переход совершается из слова $w$ в слово $w g(g$ принадлежит носителю меры $\mu$ ) с вероятностью $\mu(g)$. Реализации получившегося марковского процесса можно понимать как связные бесконечные пути на графе Кэли групшы $G$ относительно образуюших $S$, начинаюшиеся в единице групшы.

Обозначим марковскую меру на пространстве путей, соответствуюшую процессу, через $\bar{\mu}^{\infty}$. Но чаще мы будем изучать не сам процесс, а его прирашения, т.е. схему Бернулли с мерой, обозначаемой далее $\mu^{\infty}$, на пространстве бесконечных последовательностей символов из $S$ - образующих и их обратных.

Мера $\mu$ определяет элемент групповой алгебры $l^{1}(G)$, а именно,

$$
P_{\mu} f(h)=\int f(x g) d \mu(g)
$$

которьй является положительным (марковским) оператором в пространствах $l^{p}(G)$. Его спектральные свойства очень важны и мало изучены. Этот оператор также можно рассматривать в пространствах различных представлений групшы $G$. Инвариантные функции этого оператора $(P f=f)$ на группе называются гармоническими функциями.

Один из фундаментальных результатов - теорема Кестена [21] об аменабельности: спектральный радиус в $l^{2}(G)$ самосопряженного (в силу симметрии меры $\mu$ ) оператора $P_{\mu}$ равен единице в том и только том случае, когда группа, порожденная носителем меры, аменабельна.

С другой стороны, спектральньй радиус есть предел

$$
\lim _{n \rightarrow \infty} \sqrt[n]{\mu^{* n}\{e\}}
$$

которьй измеряет экспоненциальную скорость убывания вероятности возвращения траектории случайного блуждания в единицу: таким образом, для аменабельных групп и только для них скорость убьвания субэкспоненциальна. Прозрачное 
доказательство теоремы Кестена, связьвающее напрямую спектр оператора и асимптотику мощности множеств Фельнера, имеется в [8]. Подробнее основные определения и факты можно найти в статьях и книгах, см., например, [8], [51], [52].

2.3. Снос. Рассмотрим $L(g)$, длину данного элемента групшы $g$, т.е. расстояние до единицы в словарной метрике.

ОПРЕДЕЛЕниЕ 2. Сносом (drift) данной меры $\mu$ на группе называется число

$$
l_{\mu}=\lim _{n \rightarrow \infty} \frac{\mathrm{E}_{\mu^{* n}} L(g)}{n},
$$

где $\mathrm{E}_{\nu}$ - знак математического ожидания относительно меры $\nu$.

Снос есть средняя линейная (нормированная на $n$ ) скорость роста длины случайного слова, т.е. средняя скорость ухода слова на бесконечность в словарной метрике. Такая скорость сушествует для почти всех по мере $\mu^{\infty}$ бесконечных слов, это вытекает из субаддитивной эргодической теоремы Кингмана (см. лемму ниже). Поскольку нормированная длина меньше единипы, то и ее среднее по мере, т.е. снос, также существует.

2.4. Энтропия. (См. [1], [7], [8].) Определим теперь наиболее важную скалярную характеристику блуждания - энтропию пары $(G, \mu)$.

ОПРЕДЕЛЕниЕ 3. Энтропией меры $\mu$ на группе $G$ назьвается число

$$
h_{\mu}=\lim _{n \rightarrow \infty} \frac{H\left(\mu^{* n}\right)}{n}=\inf _{n} \frac{H\left(\mu^{* n}\right)}{n}
$$

где $H(\cdot)$ - обычная энтропия атомической меры.

И вновь предел существует из соображений выпуклости и совпадает поэтому с точной нижней границей. Из дальнейших свойств энтропии пока отметим лишь самые сушественные: равенство нулю энтропии равносильно тривиальности гранищы Пуассона [8] - см. далее, энтропия может вычисляться на траекториях как предел нормированных логарифмов вероятностей типичных траекторий (аналог теоремы Шеннона-Макмиллана-Бреймана [7], [11]).

Установим важное соотношение между введенными понятиями.

ТЕорема 1 (Фундаментальное неравенство). В указанных обозначениях имеет место неравенство

$$
h_{\mu} \leqslant l_{\mu} \cdot v_{S}
$$

Это неравенство было доказано в работе [42], хотя его различные частные случаи рассматривались ранее (см. работы [1], [22], библиографию в [42]); в [22] изучались блуждания на деревьях, в этом случае неравенство имеет смысл соотношений между энтропией и хаусдорфовой размерностью гармонической меры на границе, о чем упоминалось выше. Однако следует сказать, что принципиальное значение фундаментального неравенства в широком контексте теории роста в группах и полугруппах еще должно стать предметом пристального изучения.

ДокАЗАТЕльСтво. Мы фиксируем $G, S, \mu$ и опускаем поэтому индексы у $v, l, h$. 
ЛЕмма 1. Если снос отличен от нуля, то случайная последовательность длин слов удовлетворяет усиленному закону больших чисел, т.е. для любого $\varepsilon>0$ и почти любой последовательности $\omega$ существует такое $N$, что при всех $n \geqslant N$ имеет место соотношение

$$
\left|L_{n}(\omega) / n-1\right| \leqslant \varepsilon
$$

ДокАЗАтЕльство. Рассмотрим длины начальных отрезков слов как цилиндрические функционалы на бесконечном произведении $\left(G^{\infty}, \mu^{\infty}\right)$. Пусть $L_{n}(\omega)$ есть длина элемента группы, отвечающего начальному отрезку из $n$ букв бесконечной последовательности $\omega$ в алфавите $S$. Последовательность $L_{n}(\omega), n=1,2, \ldots$, как легко видеть, удовлетворяет условиям субаддитивной эргодической теоремы (Кингмана) относительно левого сдвига $T$ как преобразования $G^{\infty}$ с продакт-мерой $\mu^{\infty}$, а именHо:

$$
L_{n+m}(\omega) \leqslant L_{n}(\omega)+L_{m}\left(T^{n} \omega\right) .
$$

Поэтому последовательность $L_{n}(\omega) / n$ сходится почти всюду. То, что ее предел есть константа, следует из закона 0-1 для последовательности независимых и того, что этот предел не меняется при любом изменении конечного отрезка последовательности. У тверждение леммы равносильно сходимости по мере последовательности нормированных длин элементов и, следовательно, вытекает из сходимости почти всюду.

Перейдем к доказательству теоремы.

Удобно ввести множество $W \leqslant n$ элементов групшы, длина которых не превьшает $n$. Возьмем произвольное положительное $\varepsilon$ и выберем натуральное $n$ в соответствии с леммой. Разложим меру $\mu^{* n}$ в выпуклую комбинацию двух мер, одна из которых $-\mu_{1}$ - есть нормированное ограничение этой меры на множество $V$ тех элементов, длины которых лежат в интервале $[(1-\varepsilon) l \cdot n,(1+\varepsilon) l \cdot n]$, а другая - $\mu_{2}-$ есть нормированное ограничение меры $\mu^{* n}$ на дополнение к множеству $V$ в $W \leqslant n$. По лемме множество $V$ имеет меру больше $1-\varepsilon$, а число элементов во втором множестве не превышает общего числа элементов в $W \leqslant n$.

Тогда энтропия $H\left(\mu^{* n}\right)$ оценивается как

$$
H\left(\mu^{* n}\right) \leqslant(1-\varepsilon) H\left(\mu_{1}\right)-\ln (1-\varepsilon)+\varepsilon \cdot \ln \left(W_{\leqslant n}\right)
$$

(последнее слагаемое оценивает сверху энтропию меры $\mu_{2}$ энтропией равномерной меры на $\left.W_{\leqslant n}\right)$. Поделив на $n$ и переходя к пределу по $n$, с учетом того, что последнее слагаемое после деления на $n$ стремится к логарифмическому объему $v$, умноженному на $\varepsilon$, (см. определение), получаем

$$
h \leqslant(1-\varepsilon) H\left(\mu_{1}\right) / n+\varepsilon \cdot v .
$$

Но $H\left(\mu_{1}\right) / n$ оценивается сверху выражением $(\ln |V|) / n$, которое при $n$, стремящемся к бесконечности, дает $l \cdot v$, т.е.

$$
h \leqslant(1-\varepsilon) l \cdot v+\varepsilon \cdot v,
$$

и в силу произвольности $\varepsilon$ получаем нужное неравенство:

$$
h \leqslant l \cdot v .
$$


ЗАмЕчАниЕ 1. 1. Все рассуждения остаются верными и для полугрупп. Если рассматривать полугруппу, в которой все соотношения между образующими не меняют длину элементов (а значит, в полугруппе нет обратных элементов), то, разумеется, снос равен единице, и фундаментальное неравенство принимает вид

$$
h \leqslant v .
$$

Это относится к локально свободной полугруппе, см. §5.

2. В свободной полугруппе $n$-я свертка равномерной меры на образуюших тривиальным образом совпадает с равномерной мерой на словах длины $n$; это - тот малоинтересньй и единственньй случай, когда оба подхода к росту - обычный и динамический, тождественны уже при конечных $n$.

СлЕДСТвИЕ 1. Eсли $l_{\mu}=0$, m.е. рост слов медленнее линейного, то и әнтропия равна нулю. Тем самым, положительность энтропии влечет линейный рост длин типичных по этой мере слов.

Н. Варопоулосу [53] принадлежит важное замечание о том, что из доказанной им общей верхней оценки переходных вероятностей произвольной счетной марковской цепи можно легко вывести и нижнюю оценку для энтропии. Действительно, хотя это неравенство явно не приводится в [53], оно сразу следует из данной там основной оценки и, как отмечает автор, было поводом для ее получения. Эта нижняя оценка такова:

$$
C \cdot\left(l_{\mu}\right)^{2} \leqslant h_{\mu}
$$

здесь константа $C$ отлична от нуля и зависит лишш от группы с мерой. Таким образом, энтропия отлична от нуля одновременно со сносом. Прокомментируем это обстоятельство чуть подробнее. Легко видеть, что линейность длины типичного случайного слова с ростом $n$ приводит к линейности роста и каждой из его двух половин, четвертей и т. д., при этом коэффициент остается тем же. Отсюда по индукции можно вьвести, что вероятность типичного элемента убывает экспоненциально с ростом $n$; а тогда энтропия, которая в силу аналога теоремы Шеннона-Макмиллана-Бреймана (см. ниже) есть предел нормированного логарифма вероятности типичного слова, также положительна. Это рассуждение не дает вышеприведенной оценки, но поясняет роль линейности сноса и ее эквивалентность экспоненциальности убывания вероятностей.

Еще одно замечание относится к возможному росту длин слов. Если снос отличен от нуля, то длины почти всех слов, как утверждает лемма, растут линейно по $n$ с одной и той же скоростью. С другой стороны, хорошо известно, что на абелевых и многих других группах снос равен нулю, а скорость роста максимума длин слов сублинейна и, более точно, имеет в силу центральной предельной теоремы порядок $\sqrt{n}$. Вопрос, поставленньй в этой связи, - может ли эта скорость для каких-либо групा быть промежуточной между $\sqrt{n}$ и $n$. Этот вопрос важен для гипотетических обобщений центральной предельной теоремы для экспоненциальных групп, и до сих пор, насколько известно, он не изучался. Ответ - положителен: используя понятие размаха из классической теории случайных блужданий на решетках [54], студентка А. Г. Дюбина [55] доказала, что снос в сплетении групा $\mathbb{Z}^{2}$ и $\mathbb{Z}$ с естественными образуюшими 
имеет асимптотику: $\frac{n}{\ln n}$. Те же соображения показывают, что такой же ответ верен для свободной разрешимой группы с двумя образуюшими. Существуют и другие промежуточные асимптотики сноса. Любопытно, что при $h_{\mu}=l_{\mu}=0$, т.е. при сублинейном росте длин слов, асимптотики энтропии $H\left(\mu^{* n}\right)$ и длин могут отличаться очень сушественно, например, для абелевых групп $H\left(\mu^{* n}\right) \sim \ln n, \mathrm{a}_{\mu^{* n}} l(g) \sim \sqrt{n}$.

2.5. Сравнение систем образующих. Будем рассматривать сильно әкспоненииальные әруппь, т.е. такие, для которых существует система образующих $S$ и симметричная мера $\mu$ на ней с положительной энтропией $h_{\mu}>0$; из фундаментального неравенства следует, что $l>0$ и $v>0$, т.е. число слов растет экспоненциально. Очевидно, что положительность энтропии влечет экспоненциальность, но не влечет аменабельность (см. [8] и $\S 4$ ), а экспоненциальность не влечет положительность энтропии: например, среди экспоненциальных разрешимых групп сушествуют как сильно экспоненциальные, так и не сильно экспоненциальные. Однако, как отмечено вьше, положительность энтропии равносильна положительности сноса: $l_{\mu}>0$, т.е. линейности среднего роста длины слова.

Удобно ввести нормированную энтропию

$$
\widehat{h}_{\mu}=\frac{h_{\mu}}{l_{\mu}}
$$

Величина $\widehat{h}_{\mu}$ имеет смысл удельной әнтропии или среднего количества информаиии на одну букву (образующую).

Фундаментальное неравенство превратится в следуюшее неравенство:

$$
\widehat{h}_{\mu} \leqslant v_{S} .
$$

Эта форма фундаментального неравенства подчеркивает сходство $v_{S}$ с топологической энтропией и интерпретацию фундаментального неравенства как неравенства между метрической и топологической энтропиями. В $§ 3$ мы придадим точный смысл этой аналогии, где в роли метрической энтропии преобразования (полиморфизма) выступает энтропия $h$, а в роли его топологической энтропии - правая часть фундаментального неравенства.

Рассмотрим величину

$$
\sup _{\mu} h_{\mu} \equiv h_{S}
$$

где супремум берется по всем симметричным мерам с носителем $S$. Аналогично определяются величины $\widehat{h}_{S}$ и $l_{S}$.

ЛЕмма 2. Существует мера с носителем $S$, для которой реализуется супремум $h_{S}$ величины $h_{\mu}$.

Действительно, так как множество $S$ конечно, то множество симметричных мер на нем компактно. Энтропия $h_{\mu}$, как можно убедиться с помощью простых оценок, приведенных в [42], непрерывно зависит от меры $\mu$. 
Поскольку различные меры $\mu$ определяют попарно взаимно сингулярные бернуллиевские меры на пространстве бесконечных слов (см. следующий пункт), то непрерьвность математических ожиданий функционалов, зависящих от $n$ (таких как нормированная длина слова), вообще говоря, не имеет места. Поэтому пока неясно, сушествует ли мера с носителем $S$, реализуюшая супремумы для величин $l_{\mu}$ и $\widehat{h}_{\mu}$, но для дальнейшего важна как раз мера, реализуюшая супремум величины $h_{\mu},-$ эта мера называется мерой с максимальной энтропией на данной системе образующих $S$. Из предыдущего видно, что имеет место неравенство:

$$
\widehat{h}_{S} \leqslant v_{S},
$$

и

$$
h_{S} \leqslant l_{s} \cdot v_{S} .
$$

Стоит отметить, что равномерная мера на образуюших $S$ не обязательно является мерой с максимальной энтропией, так как образующие могут быть неравноправны с точки зрения определяющих соотношений. Если же существует транзитивно действующая на образующих группа, продолжающаяся до автоморфизма всей группы, то естественно предположить, что равномерная мера на образуюших и обратных к ним есть мера с максимальной энтропией.

Наконец, введем величину

$$
q_{S} \equiv \frac{\widehat{h}_{S}}{v_{S}}
$$

Величина $q_{S}$ зависит только от системы образуюших $S$ и, как мы увидим, является ее важной характеристикой.

ОПРеДЕЛЕниЕ 4 . Система образуюших $S$ в сильно экспоненциальной группе $G$ называется экстремальной, если величина $q_{S}$ принимает максимально возможное значение $q(G)=q=\sup q_{S}$ (супремум берется по всем конечным системам образующих).

Из неравенства следует, что

$$
q \leqslant 1 .
$$

Возникает возможность сравнения систем образующих с помощью параметра $q_{S}$. Неизвестно, в каждой ли конечно-порожденной группе сушествует экстремальная система образуюших. Однако введенное вьшше число $q(G)$ является нетривиальным инвариантом конечно-порожденной сильно әкспоненциальной группы.

ОПРеДЕЛЕНИЕ 5. Группа $G$ назьвается свободноподобной, если $q(G)=1$.

Смысл термина разъясняется из следуюшего простого и известного вычисления, показьваюшего, что в свободной группе с $d>1$ образуюшими система свободных образуюших экстремальна, а равномерная мера на образуюших имеет максимальную энтропию, т.е. $q=1$. Явные значения всех трех констант для этой меры таковы:

$$
v=\ln (2 d-1), \quad l=\frac{d-1}{d}, \quad h=\frac{(d-1) \cdot \ln (2 d-1)}{d} .
$$

Эти подсчеты хорошо известны, их простота определяется тем, что в этом примере сами длины случайных слов образуют марковскую цеп, а именно, простое случайное 
блуж дание на одномерной полурешетке с отражением в нуле. В конкретных примерах наибольшую трудность представляет подсчет энтропии, главный метод здесь - теорема шенноновского типа, позволяюшая считать энтропию на траекториях.

Нетрудно убедиться в том, что конечные расширения свободноподобных групп свободноподобны, поэтому виртуально свободные групшы (конечные расширения свободных групп) и, в частности, свободное произведение циклических групп суть также свободноподобные группы (напомним, что только свободное произведение двух групп второго порядка не является виртуально свободной группой). Но и явные вычисления для свободных произведений циклических групп, как и для некоторых подгрупп $S L(2, \mathbb{Z})$, дают равенство.

Теперь дадим интерпретацию фундаментального неравенства с точки зрения генерации элементов группы, повторяя чуть более подробно то, о чем шла речь во введении. Как оценить, какая система образующих группы более удобна для работы? Мы можем говорить, например, о нормальных формах элементов группы в данных образуюших и об их сравнениях. Но следует помнить, что нормальные формы могут не существовать в алгоритмическом смысле, так как их сушествование и означает возможность алгоритмического решения проблемы тождества в группе. Но даже в тех классических случаях, когда такая форма есть, она может быть неустойчивой, т.е. сушественно меняться при малом изменении элемента групшы, т.е. умножении его на какую-либо образуюшую.

Динамический же подход состоит в другом - мы порождаем элементы случайным образом в соответствии с той или иной мерой на образуюших и затем следим, насколько типичны порождаемые элементы в группе. Точная формулировка состоит в том, что сравниваются сверточные степени выбранной меры с равномерной мерой на словах данной длины. Действительно, распределение элементов группы, которое мы получим в процессе генерирования после $n$ шагов, есть в точности $n$-я свертка исходной меры, а снос показывает, какова их средняя длина; поэтому сравнивать этот массив надо с равномерной мерой на словах той же длины. Таким образом, фундаментальное неравенство показывает, в какой степени эти меры близки, - если мы имеем строгое неравенство, то это означает, что случайный процесс порожсдет әкспоненциально малую часть множсества всех слов. Если же оно преврашается в равенство, то случайное генерирование дает основную часть всех элементов группы (отличаюшуюся лишш на субэкспоненциальную поправку). Поэтому следует сравнивать системы образующих по показателю $q_{S}$, введенному выше, - он характеризует отклонение между равномерной мерой и динамической, а экстремальная система образуюших $\left(q_{S}=q(G)\right)$, если она сушествует, - дает наилучшее возможное для данной группы решение вопроса. В некоторых примерах константа $q_{S}$ для естественной системы образуюших меньше единицы, но не известно, является ли эта система образующих экстремальной. Именно такое положение сейчас с группой кос - мы имеем лишш частичную информацию о росте и фундаментальном неравенстве для произвольных образующих в ней.

Следует отметить еще одно общее соображение по поводу случайного генерирования элементов групп, о котором шла речь вьше: можно, конечно, генерировать элементы не независимым образом, а следуя, например, марковскому правилу, частично учитывающему соотношения между образующими и запрещающему ставить после какой-либо образуюшей некоторую другую и т. д. Это приводит к более сложным по- 
становкам вопроса, и проблема явного описания границы и гармонических функций остается и в этом случае.

Вышеприведенные определения ставят ряд вопросов, некоторые из которых приведем здесь.

Главный вопрос таков:

1. В любой ли конечнопорожденной сильно экспоненциальной группе сушествует экстремальная система образующих, т.е. всегда ли $q(G)=1$ ?

Иначе говоря, всякая ли конечнопорожденная группа свободноподобна?

Другие вопросы:

2. Описать меры с максимальной энтропией для фиксированной системы образующих.

3. Когда такая система образуюших единственна в существенном?

Некоторый материал для анализа этих, по-видимому, сложных вопросов дает содержание следуюших параграфов. В них мы привлекаем внимание к некоторым классам групп, которые не изучались до сих пор в этом контексте.

\section{$\S$ 3. Структура границ и связь фундаментального неравенства с полиморфизмом границы}

В этом параграфе мы исследуем предельные объекты - гранищы случайных блужданий, структуры на них - и придаем смысл введенным раннее характеристикам в терминах этих предельных пространств и их преобразований, мы приводим также обший способ описания границ для групп со стабильной нормальной формой.

3.1. Бернуллиевская модель и описание различных границ (границавыход, стационарная граница, граница Пуассона-Фюрстенберга, гармонические функции). Мы изучаем статистику слов в группе, границы и др. с помощъю модели бесконечных слов и разбиений пространства бесконечных слов. С точки зрения случайных блужданий эта модель рассматривает прирашения марковского процесса вместо самого процесса. Такой подход ближе к эргодической теории и теории меры, более того, основная идея дальнейшего - свести все вопросы о случайных блужданиях к тем или иным задачам об автоморфизме Бернулли. Сушественную роль при этом играют убываюшие последовательности разбиений на этих пространствах.

Пусть $S^{\mathbb{N}}$-пространство бесконечных слов в алфавите $S=\left(g_{1}, \ldots, g_{k}, g_{1}^{-1}, \ldots, g_{k}^{-1}\right)$ - образуюших группы $G$. В терминах графа Кэли, соответствуюшего системе образуюших $S$, пространство $S^{\mathbb{N}}$ есть пространство бесконечных связных путей, начинаюшихся из единицы группы, каждое ребро этого пути занумеровано элементом $S$. В следующем параграфе мы рассмотрим пространство путей на графе Кэли любой конечнопорожденной групшы как пространство путей на решетке, что даст новые способы изучения случайных блужданий.

Если $\mu$ - симметричная мера на образуюших, то $\mu^{\infty}$ - бернуллиевская мера (продакт-мера) на $S^{\mathbb{N}}$. Все последуюшие разбиения понимаются как измеримые разбиения этого пространства Лебега $\left(S^{\mathbb{N}}, \mu^{\infty}\right)$. В то же время на $S^{\mathbb{N}}$ определена стандартная вполне несвязная компактная топология, а левый сдвиг есть непрерывный эндоморфизм этого компакта, сохраняющий меру.

Рассмотрим сначала координатные (цилиндрические) разбиения $\eta_{m}$ пространства $\left(S^{\mathbb{N}}, \mu^{\infty}\right)$ на последовательности с фиксированной $m$-й координатой. Разбиение 
$\xi_{n}^{0}=\bigvee_{m \geqslant n} \eta^{m}$ назовем $n$-хвостовьм - его элемент есть множество всех последовательностей с фиксированными координатами, начиная с $(n+1)$-й. Разбиения $\xi_{n}^{0}$ убывают, и их измеримое пересечение $\bigwedge \xi_{n}^{0}$ есть - по закону 0-1 Колмогорова - тривиальное разбиение пространства $\left(S^{\mathbb{N}}, \mu^{\infty}\right)$. Теоретико-множественное ("неизмеримое") пересечение $\bigcap_{n} \xi_{n}^{0}$ разбиений $\xi_{n}^{0}$ есть разбиение на классы последовательностей, различаюшихся на произвольном конечном числе мест. Оно “абсолютно неизмеримо", а его измеримая оболочка, т.е. измеримое пересечение последовательности $\left\{\xi_{n}\right\},-$ тривиальна. Определения всех этих разбиений не используют структуру группы.

Определим теперь последовательность конечных цилиндрических измеримых разбиений $\left\{\zeta_{n}(G)\right\}$, относя в один элемент разбиения $\zeta_{n}(G)$ все последовательности из $S^{\mathbb{N}}$, у которых произведения в группе $G$ первых $n$ координат одинаковы. Мы будем опускать указание на группу в обозначении $\zeta_{n}$, если нет опасности смешения.

Очевидно, что энтропия конечного разбиения $\zeta_{n}$ равна $H\left(\mu^{* n}\right)$, а число его элементов есть число элементов группы длины $n$. Поэтому следуюшие равенства тавтологичны:

$$
\begin{gathered}
h_{\mu}=\lim _{n} \frac{H\left(\zeta_{n}\right)}{n}, \\
v=\lim _{n} \frac{\ln \left|\zeta_{n}\right|}{n},
\end{gathered}
$$

здесь $|\cdot|$ - число элементов в разбиении. Что касается интерпретации сноса, то в каждом элементе разбиения $\zeta_{n}$ есть слово минимальной длины, следовательно, оно определяет функцию длины $l_{n}$ на $S^{\mathbb{N}}$, а ее математическое ожидание есть средняя длина слова, нормированньй на $n$ предел этого ожидания и есть снос. Гораздо более важная интерпретация этих констант и фундаментального неравенства будет дана ниже.

Разбиения $\zeta_{n}$ не образуют возрастающей последовательности, и их произведение не является инвариантньм относительно левого сдвига. Для наших целей более удобна несколько иная последовательность измеримых разбиений $-\left\{\xi_{n}\right\}$, определяемая следующим образом:

$$
\xi_{n}=\zeta_{n} \vee \xi_{n}^{0}
$$

Разбиения $\xi_{n}$ имеют конечные элементы, состояшие из последовательностей, все координаты которых начиная с $(n+1)$-й совпадают, а произведения первых $n$ координат в группе $G$ - одинаковы. Очевидно, что каждое разбиение $\xi_{n}^{0}$ мажорирует разбиение $\xi_{n}$. Имеет место следующая очевидная лемма.

ЛЕмма 3. Введенные разбиения связаны следующим образом:

$$
\xi_{n}=\bigvee_{m>n} \zeta_{m}
$$

при этом последовательность разбиений $\left\{\xi_{n}\right\}$ монотонно убывает, а ее измеримое пересечение, обозначаемое далее $\sigma(G, \mu)$, совпадает с верхним пределом разбиений $\zeta_{n}$ :

$$
\bigwedge_{N} \bigvee_{m>N} \zeta_{m}=\bigwedge_{n} \xi_{n}=\sigma(G, \mu)
$$


Это разбиение, вообще говоря, неинвариантно относительно левого сдвига.

Иног да удобнее говорить о разбиениях как об отношениях эквивалентности. В этом смысле мы имеем монотонные последовательности отношений эквивалентности бесконечных слов, сходящиеся к некоторому предельному отношению эквивалентности, однако необходимо рассматривать не само предельное отношение, а его измеримую оболочку.

ОПРЕДЕЛЕНИЕ 6. Назовем измеримое пересечение

$$
\bigwedge_{n} \xi_{n}=\sigma(G, \mu)
$$

пространства $\left(S^{\mathbb{Z}}, \mu^{\infty}\right)$ граничным разбиением, отвечающим мере $\mu$, а фактор-пространство по нему, $S^{\mathbb{N}} / \sigma,-$ границей пары $(G, \mu)$. Образ меры $\mu^{\infty}$ на фактор-пространстве обозначим $\mu_{\sigma}$.

Более точно было бы сказать, что это граница-выход марковского процесса, отвечающего случайному блужданию, если перейти к соответствующему марковскому процессу. В нашей ситуации граница совпадает со стационарной границей (см. ниже), границей Пуассона и др. (см. [8]). Для группы движений пространства Лобачевского - это абсолют плоскости и т. д. Гранишы представляют основной интерес в теории случайных блужданий на группах и гармоническом анализе. Сложность их изучения в тех случаях, когда она нетривиальна, в том, что нет единого способа определить цилиндрический базис разбиения $\pi$ - он существует, только если существует стабильная нормальная форма элементов групшы, как будет видно из дальнейшего, а такая нормальная форма существует, по-видимому, не всегда.

Для абелевых групп (и симметричных мер на них) граница тривиальна, и это есть следствие другого закона 0-1 - закона Хьюитта-Сэвиджа, по которому сигма-алгебра измеримых подмножеств пространства Бернулли, инвариантных относительно группы финитных подстановок, тривиальна, иначе говоря, бесконечная симметрическая группа действует на этом пространстве эргодично. Таким образом, всякая группа с мерой, для которой граница тривиальна (например, группь субэкспоненциального роста), задает свой закон 0-1 - мы получаем алгебраический источник нетривиальных 0-1-законов для классических бернуллиевских последовательностей. Особенно интересны те из них, которые возникают из экспоненциальных групп, граница которых тривиальна, как, например, для свободной разрешимой группы с двумя образуюшими ступени два (см. $\S 4)$.

Введем еще одно важное семейство разбиений. Зафиксируем два натуральных числа $(n, k)$ и скажем, что две точки $x, y \in S^{\mathbb{N}}(n, k)$-эквивалентны, если произведение первых $n$ координат точки $x$ совпадает с произведением первых $k$ координат точки $y$ и для всех $i x_{n+i}=y_{k+i}$. Тем самым, определено разбиение $\zeta_{n, k}$. При $n=k$ получаем разбиения $\zeta_{n}$.

ОПРЕДЕЛЕНИЕ 7. Измеримое пересечение разбиений

$$
\bigwedge_{n, k} \zeta_{n, k}
$$


обозначается $\pi(G, \mu)$ и называется стационарным разбиением, фактор-пространство $\Gamma(G, \mu)$ - стационарной границей пары $(G, \mu)$, а фактор-мера $\mu_{\pi}-$ гармонической мерой.

Стационарная граница естественно отображается на границу, так как стационарное разбиение мажорируется граничным, ниже мы увидим, что в нашем случае это отображение есть изоморфизм.

ТЕОрема 2. Отображение стационарной границы на границу есть изоморфизм соответствующих пространств с мерой.

Доказательство можно провести в “бернуллиевских" терминах, но на самом деле это утверждение хорошо известно в обычной теории марковских цепей; кратко опишем его.

Прежде всего отметим, что при переходе от марковской цепи случайных блужданий к независимым приращениям, т.е. к бернуллиевской схеме, обычным понятиям стационарной границы и границы-выход для марковской цепи (см. [51], [8]) соответствуют данные выше определения стационарной границы и границы. Иначе говоря, факторизуемые пространства различны, но фактор-пространства канонически отождествлены: отображение, переводящее траекторию блуждания в последовательность прирашений, согласовано с обоими отношениями эквивалентности: две траектории, эквивалентные в смысле стационарного отношения эквивалентности, имеют прирашения, эквивалентные в том же смысле, то же самое верно и для хвостового отношения. Далее нужно повторить хорошо известное рассуждение из теории стационарных марковских цепей: всякая апериодическая марковская иепь с конечным или счетным множеством состояний регулярна, т.е. удовлетворяет закону 0-1 и ее граница-выход - тривиальна. Апериодичность означает отсутствие разбиения множества состояний на собственные подмножества (подклассы), которые детерминированно переставляются при сдвиге. Таким образом, апериодичность, эквивалентная эргодичности, т.е. тривиальности стационарной границы, влечет тривиальность границы-выход. Этот вывод верен и для сигма-конечной инвариантной меры. Утверждение перестает быть верным для произвольного (континуального) пространства состояний: известны примеры стационарных эргодических, но нерегулярных марковских цепей с непрерывным множеством состояний (ср. понятие сверхгрубости гиперболических систем в [56], [57]). Изоморфизм гранишы и стационарной границы следует из "условного" аналога приведенного рассуждения (т.е. примененного к условному процессу, а именно, эргодической компоненте). Теперь мы можем отождествить обе границы как пространства с мерами и обозначать их одинаково - $(\Gamma(G, \mu), \widehat{\mu})$, и называть просто границей, а предельные разбиения (граничное и стационарное), по которьм факторизуется пространство $S^{\mathbb{N}}$, обозначать единьм образом - $\sigma(G, \mu)$, опуская иногда упоминание о группе $G$ и о мере $\mu$, и назьвать граничным разбиением, а меру $\widehat{\mu}$ - гармонической мерой.

Точки границы фактически классифицируют типы асимптотических поведений траекторий блуждания при стремлении времени к бесконечности, различимые измеримыми функционалами. Мы отсылаем к специальным статьям тех, кто интересуется теорией границ и ее многочисленными связями с уравнениями, пополнениями груп, теорией конщов групп и т. д. 
В теории марковских процессов и операторов определяется наиболее известная из границ - граница Пуассона, которая впервые изучалась в общем групповом контексте Г. Фюрстенбергом [3], [4], чем и объясняется предложение называть ее границей Пуассона-Фюрстенберга. О понятиях и теории $\mu$-гармонических функций имеется колоссальная литература (см., например, [51]).

Каждая точка $\gamma \in \Gamma(G, \mu)$ стационарной границы (в вьшеприведенном смысле) есть по определению элемент разбиения $\sigma$, на почти каждом из которых определена условная мера. Возьмем значение этой условной меры на каком-либо элементе разбиения $\zeta_{n}$, этот элемент определяет некоторый элемент групшы $h \in G$, обозначим его через $f_{\gamma}(h)$, тем самым, определена функция на группе. Ее значение в единице группы равно единице, и из определения следует, что $f_{\gamma}$ есть положительная ограниченная гармоническая функиия

$$
P_{\mu} f_{\gamma}=f_{\gamma}
$$

Особую роль в дальнейшем играют значения гармонической функции на множестве $S$ - образующих и их обратных $-f_{\gamma}(g)$, т.е. значения условной меры на элементах разбиения $\zeta_{1}=\eta_{1}$.

Таким образом, определено mod 0 отображение стационарной границы на множество положительных ограниченных гармонических функций относительно оператора перехода $P_{\mu}$, определяемого мерой $\mu$. Гармоничность функции и корректность определения следуют из стационарности.

ЛЕмма 4. Это отображсние есть изоморфизм mod 0 межсуу стационарной границей и границей Шоке множества ограниченных нормированных положительных гармонических функиий.

Тем самым, стационарная граница $(\Gamma(G, \mu), \widehat{\mu})$ совпадает с границей ПуассонаФюрстенберга. Всякая положительная ограниченная гармоническая функция представляется интегралом по стационарной границе. Тривиальность границы Пуассона для абелевых групп есть содержание теоремы Шоке-Дени (см. [51]). В одном из следующих пунктов мы используем гармонические функции для определения нового марковского преобразования (полиморфизма) границы. Несколько более тонкий вопрос - как все эти определения перенести из метрической в топологическую категорию; проблема состоит в том, что рохлинская теория условных мер гарантирует их существование не всюду, как можно было бы предположить в некоторых случаях, а лишш почти всюду, поэтому и гармонические функции соответствуют почти всякой, а не всякой точке гранищы, вообше говоря. Тем не менее, запас корректно определенных гармонических функций задает обычньм образом топологию на границе.

Разбиения $\pi, \sigma$ можно строить в пространстве траекторий произвольного одностороннего стационарного процесса. В обшем случае (небернуллиевского пространства) эти разбиения аналогичны разбиениям соответственно на траектории группы финитных замен координат $(\sigma)$ и на траектории (по Рохлину) полугруппы сдвигов $(\pi)$. Разумеется, оба разбиения и оба фактор-пространства или границы, вообще говоря, не совпадают, поскольку различны понятия эргодичности и регулярности по Колмогорову: тривиальность $\sigma$ означает регулярность (обьчньй закон 0-1), а тривиальность $\pi$ - эргодичность. Эти разбиения встречаются также в алгебраической теории динамических систем при построении $C^{*}$-алгебр, связанных с эндоморфизмами. 
Существенно при этом, что эти конструкции относятся к пространству односторонних последовательностей, в котором действует односторонний сдвиг. В связи с этим упомянем еще об одном естественном построении - “двусторонизации". Рассмотрим пространство $S_{f}^{\mathbb{Z}}$ - пространство бесконечных в обе сторонь последовательностей символов, причем лиш конечное число координат с отрицательными номерами отлично от единицы групшы (можно считать, что единица входит в $S$, но может встречаться только на местах с отрицательными номерами). Это пространство можно представить как объединение счетного числа экземпляров $S^{\mathbb{N}}$, занумерованных конечными последовательностями, стояшими на местах с отрицательными номерами. Определим сигма-конечную меру на этом пространстве, приписав каждому из этих экземпляров меру 1 , а на $S^{\mathbb{N}}$ определим ее, как и выше. Разбиения $\bar{\xi}_{n}, n \in \mathbb{Z}$, определяются на $S_{f}^{\mathbb{Z}}$ точно так же - а именно, элемент есть множество двусторонних последовательностей, совпадаюших начиная с координаты с номером $n+1$ и имеющих одинаковое произведение в группе всех предшествующих координат (число отличных от единицы - конечно). Польза этой конструкции в том, что теперь пересечение $\bigwedge_{n} \bar{\xi}_{n}$ есть уже инвариантное относительно сдвига разбиение, а сдвиг сохраняет введенную сигма-конечную меру. Произведение координат с отрицательными номерами можно воспринимать как начальную точку в нулевой момент времени при случайном блуждании - в основной модели мы всегда считали ее единищей группы, что лишает, конечно, модель инвариантности. Недостаток этой конструкции - в бесконечности инвариантной меры, поэтому теория гранищ не становится проще от такого перехода.

Вернемся к одностороннему случаю. Приведенные определения разбиений естественно обобшаются на широкий класс марковских процессов - блужданий на ассоциативных системах, формальных грамматиках и др. Вот точное определение. Пусть $S$ - произвольный алфавит, и пусть для каждого $n$ задано разбиение $\theta_{n}$ множества слов длины $n$ на эквивалентные слова (синонимы), при этом при разных $n$ они согласованы следуюшим образом: если два слова длины $n+k$ эквивалентны (относительно разбиения $\theta_{n+k}$ ) и их начала (или концы) длины $k$ также эквивалентны (относительно разбиения $\theta_{k}$ ), то и оставшиеся части длины $n$ также эквивалентны (относительно $\theta_{n}$ ). Теперь мы можем повторить построение разбиений $\xi_{n}$ на $S^{\mathbb{N}}$, определить обе границы, расширенное пространство и т. д. Условие согласования разбиений $\theta_{n}$ гарантирует монотонность последовательности $\xi_{n}$ на $S^{\mathbb{N}}$. Пересечение $\bigwedge_{n} \xi_{n}$ неинвариантно относительно левого сдвига, но обладает более слабым свойством, а именно, полуинвариантностью: т.е. является инвариантным относительно преобразований, сдвигающих последовательность на один шаг вправо и ставящих на первое место фиксированную образуюшую. Иначе говоря, вместо условия инвариантности: $T^{-1} \xi \prec \xi \prec T^{-1} \xi \vee \eta_{1}$ вьполнено условие полуинвариантности: $\xi \prec T^{-1} \xi \vee \eta_{1}$.

3.2. Проблема описания границ. Явное описание границ составляет основной предмет теории асимптотического поведения случайных блужданий и гармонического анализа на группах. Канонический алгебраический пример нетривиальной границы - граница Мартина свободной групшы для случайного блуждания по образующим и обратным к ним, найденная в работе Дынкина-Малютова - см. [24]. Ею является пространство бесконечных односторонних несократимых слов. Оно же (уже как пространство с гармонической мерой) выступает в качестве границы Пуассона-Фюрстенберга для блужданий с переходной мерой с конечным носителем (и даже для мер с 
первым моментом - см. ссылки в [58]). Особенность этого примера в том, что границу, являющуюся по определению фактор-пространством, удается вложить в исходное пространство, т.е. построить сечение к факторизации по граничному разбиению. Как уже говорилось, это можно сделать далеко не всегда, так как наличие сечения связано с сушествованием стабильной нормальной формы в группе (см. определение далее). Имеются немногочисленные примеры явных вычислений, их можно найти в работах Фюрстенберга [3]-[5], а из последних работ см. [25]-[27]. Ниже в этом и последующих параграфах будут даны новые примеры.

Двойственньм к вопросу об описании границ является вопрос о том, как охарактеризовать те функции от бесконечных траекторий, которые постоянны на элементах разбиения $\sigma=\pi$ и, тем самым, зависят лиш от точек границы $(\Gamma, \mu)$. Докажем общее утверждение, играющее ту же роль для вычисления границ, что и эргодический метод [59] для нахождения инвариантных мер для ручных отношений эквивалентности. Мы используем обозначения, введенные вьше. Для определенности мы будем говорить об $L^{2}$-функциях.

ТЕОРема 3. Всякая функиия $F$ на $S^{\mathbb{Z}}$, постоянная на элементах разбиения $\sigma$ (т.е. корректно определенная на границе), есть предел в $L^{2}$ последовательности функций $\left\{f_{n}\right\}$, где $f_{n}$ измерима относительно разбиения $\zeta_{n}$.

Напомним, что разбиения $\zeta_{n}$ - цилиндричны, но не образуют возрастающую последовательность разбиений. В дальнейшем мы обозначаем (конечномерное) пространство функций, измеримых относительно $\zeta_{n}$, через $B_{n}$, а подпространство пространства $L^{2}$ функций, измеримых относительно разбиения $\sigma$, т.е. функций на границе, через $L^{2}(\Gamma, \widehat{\mu})$. Наше утверждение сводится к тому, что это подпространство есть предел подпространств $B_{n}$.

ДокАЗАТЕльство. Пусть $F$ - произвольная измеримая относительно $\sigma$ функция. Для всякого $\varepsilon>0$ найдется $n(\varepsilon)=n$ и цилиндрическая функция $F_{n}$, отличающаяся по норме $L^{2}$ от $F$ не более чем на $\varepsilon$. Возьмем условное ожидание от обеих функций относительно разбиения $\xi_{n}$; это не изменит функцию $F$, поскольку она измерима относительно $\xi_{n}$ (напомним, что $\xi_{n} \succ \sigma$ ), а в силу цилиндричности $F_{n}$ и бернуллиевости меры $\mu^{\infty}$ условное ожидание от $F_{n}$ снова будет цилиндрической функцией и потому измеримой относительно $\zeta_{n}$. Так как условное ожидание не увеличивает норм, то теорема доказана.

Таким образом, вычисление границы сводится $к$ нахождению всех пределов функиий, измеримых относительно иилиндрических разбиений $\zeta_{n}$ или, иначе, к нахождению пределов функций от слов в алфавите $S$ длины $n$, зависящих лишь от класса слова, т.е. от соответствуюшего элемента группы. Несмотря на почти тавтологичность этого утверждения, оно играет основную роль в обосновании вычислений границ. Конечно, само нахождение пределов есть конкретная задача в конкретной групповой ситуации, однако мы увидим, что есть весьма общие классы групп, где и это вычисление может быть стандартизировано.

Специальным случаем общей проблемы является задача распознавания тривиальности гранишы. В этом случае предельные функции - лишш константы. Хотя энтропийный критерий нетривиальности границы [8], [11], [60], обсуждаемьй с новой точки зрения далее, весьма эффективен, тем не менее подсчет энтропии все же непрост, 
и иногда нетривиальность гранищы проще доказать прямым предъявлением нетривиальных хвостовых множеств или непостоянных гармонических функций, а не вычислением энтропии. Аналогично этому, и в эргодической теории проверка того, положительна ли энтропия конкретного автоморфизма и эргодичен ли он, часто представляет содержательную проблему.

Очевидно, что проблема распознавания тривиальности гранищы для равномерных мер на образуюших по заданньп соотношениям между образуюшими, т.е. выяснения равна ли нулю энтропия, - алгоритмически неразрешима, как любая содержательная проблема теории групп (см., например, [61], [62]). Явное описание границ $\Gamma(G, \mu)$ имеется лишь для небольшого количества счетных групп и мер на них. Помимо свободных групп, фундаментальных групп поверхностей [27] и классических симметрических пространств [4], [8] таких примеров совсем немного.

Как правило, пространство, являющееся "кандидатом" в границы, угадывается легко; сложнее доказать, что это пространство совпадает с гранищей. Иначе говоря, первый относительно простой шаг состоит в нахождении запаса некоторых хвостовых функций на пространстве траекторий блужданий (или функций на $S^{\mathbb{N}}$, измеримых относительно разбиения $\sigma$ ) - это дает, вообше говоря, лишь фактор-пространство гранишы, а второй шаг (если правильно угадан кандидат) - в доказательстве того, что они исчерпьвают весь запас функций на границе, т.е. что граница условного процесса при фиксации точки из фактор-пространства тривиальна, или, эквивалентным образом, что все пределы исчерпьваются указанными функциями. В ряде примеров первьй шаг был сделан давно (сплетения, лампочные группы). Ниже мы используем вышеприведенную теорему для того, чтобы сделать второй шаг в конкретных случаях.

Начнем со следуюшего определения.

ОПРЕДЕЛЕНИЕ 8. Нормальной формой элементов групшы относительно данной системы образующих $S$ назьвается отображение, сопоставляющее каждому элементу группы некоторую запись этого элемента в виде слова в алфавите $S$. Минимальной назьвается нормальная форма, в которой длина слова равна длине элемента. Нормальная форма назьвается стабильной относительно меры $\mu$ на $S$, если для почти всякой по мере $\mu^{\infty}$ бесконечной последовательности $\left(z_{1}, z_{2}, \ldots\right)$ как элемента пространства $\left(S^{\mathbb{N}}, \mu^{\infty}\right)$ последовательность нормальных форм отрезков этой последовательности стабилизируется, т.е. сходится в слабой топологии к некоторому элементу того же пространства.

Нетрудно дать количественный критерий стабильности нормальной формы для равномерных мер.

Лемма 5. Пусть в группе $G$ выцелена система образующих $S=\left\{g_{1}, \ldots, g_{k}\right.$, $\left.g_{1}^{-1}, \ldots, g_{k}^{-1}\right\}$ и $\mu$-равномерная мера на $S$. Для того чтобъ некоторая нормальная форма относительно образующих $S$ била стабильной относительно равномерной мерь $\mu$, необходимо и достаточно, чтобъ

$$
\lim \frac{W\left(N, k_{N}\right)}{W_{N}}=1
$$

где $W_{N}-$ число әлементов в группе, длина данной нормальной формы которьх в образующих $S$ равна $N, W(N, k)$ - число тех из них, у которых первые $k$ знаков 
данной нормальной формы не меняются при умножении әлемента справа на любой из әлементов из $S$, а $k_{N}$ - произвольная стремящаяся $\kappa$ бесконечности при $N \rightarrow \infty$ последовательность натуральных чисел.

Если существует стабильная нормальная форма, то в пространстве $S^{\mathbb{N}}$ выделено подмножество элементов, являющихся пределами нормальных форм почти всех элементов. Такие элементы по аналогии со свободной группой мы будем назьвать бесконечными несократимыми словами. Отметим, что их запас определяется не только выбором образующих, но и выбором нормальной формы. Обозначим отображение, сопоставляющее почти каждому элементу $\left(S^{\mathbb{N}}, \mu^{\infty}\right)$ его бесконечное несократимое слово, через $\Phi$, а его образ через $\left(\Gamma_{0}, \widehat{\mu}_{0}\right)$. Мы будем использовать следующие обозначения: если элемент $z \in S^{\mathbb{N}}$, т.е. последовательность $z=\left\{z_{i}\right\}, i \in \mathbb{N}$, стабилизируется, то его образ $\Phi(z)$ обозначается через $\left\{\widehat{z}_{i}\right\}, i \in \mathbb{N}$, а его координаты называются стабильнылми координатами әлемента $z$ (точнее было бы называть их $\infty$-стабильными). Координаты нормальной формы начального отрезка последовательности $\left\{z_{i}\right\}, i \in\{1, \ldots, n\},\left\{\widehat{z}_{i}^{n}\right\}$ будем назьвать $n$-стабильными - их число может быть меншше $n$, но мы будем считать его равным $n$, добавляя, если нужно, к слову несколько единищ справа. По определению стабилизация означает, что для любого $i$, начиная с достаточно большого $n$, значения $\widehat{z}_{i}^{n}$ не меняются, т.е. при любом $i$ стабильная $i$-я координата совпадает с $n$-стабильной при больших $n$.

У тверждение следуюшей леммы непосредственно вытекает из стабильности.

ЛЕмма 6. Если нормальная форма стабильна, то относительно гармонической меры координаты бесконечных несократимых слов асимптотически независимы, точнее, распределение пары образующ,их, стоящих на местах $k u k+N$, стремится $к$ прямому произведению при $N \rightarrow \infty$.

Действительно, стабилизация равносильна тому, что на множестве меры, сколь угодно близкой к единице, первые $k$ координат не меняются, и потому условное распределение при условии фиксации $k$-й координаты будет стремиться к безусловному. Аналогично этому, асимптотическая независимость имеет место не только для координат, но и для отрезков несократимых слов.

ТЕОрема 4. Если для пары $(G, \mu)$ группь $G$ с мерой $\mu$ с конечнылм носителем существует стабильная нормальная форма, то граница $(\Gamma(G, \mu), \widehat{\mu})$ совпадает с $\left(\Gamma_{0}, \widehat{\mu}_{0}\right)$ - множеством бесконечных несократимых слов, снабженном мерой-образом.

ДокАЗАТЕЛЬСТво.Будем использовать вышеприведенный метод отыскания функций на $L^{2}\left(S^{\mathbb{N}}, \mu^{\infty}\right)$, измеримых относительно разбиения $\sigma$, т.е. функций на границе. Подпространство $B_{n}$ в нашем случае есть подпространство $n$-цилиндрических функций в $L^{2}\left(S^{\mathbb{N}}, \mu^{\infty}\right)$, зависяших лишь от нормальной формы $n$-отрезка бесконечного слова. Эта нормальная форма имеет длину $n$ (с добавленными единицами, если нормальная форма короче $n$ ) и, вообшеговоря, не совпадает с отрезком длины $n$ соответствующего бесконечного несократимого слова. Таким образом, функции из $B_{n}$ есть функции от элементов группы длины, не превосходящей $n$, заданных в нормальной форме. Наша цель - доказать, что всякая $L^{2}\left(S^{\mathbb{N}}, \mu^{\infty}\right)$-функция, измеримая относительно $\sigma$ (т.е. функция, корректно определенная на границе $\Gamma(G, \mu))$, есть функция от стабильных координат (коротко - стабильная функция). Иначе говоря, требуется установить, что 
не существует пределов функций из $B_{n}$, которые были бы ортогональны всем стабильным функциям. Мы несколько усилим условие ортогональности и докажем сначала более слабое утверждение: не существует непостоянных предельных функций, независимых со всеми стабильными функииями. Заметим, что из теоремы Рохлина о классификации измеримых разбиений (и это главньй ее случай) следует, что независимое дополнение существует у всякого разбиения, почти все элементы которого непрерывны, или более общо - изоморфны. Это свойство в свою очередь вытекает из наличия эргодически действующей группы, оставляющей разбиение инвариантным. В нашем случае это можно вывести из групповых соображений, поэтому наше предположение не умаляет обшности. Но способ доказательства годится и для произвольного дополнительного разбиения (не независимого).

Пусть $F$ - предельная функция, независимая со всеми стабильными функциями. Возьмем убывающую, стремяшуюся к нулю последовательность положительных чисел $\delta_{n}$ и будем строить аппроксимацию функции $F$ функциями из $\left\{B_{n}\right\}$.

По $\delta_{1}>0$ выберем натуральное $n_{1}$ и цилиндрическую функцию от $n_{1}$ координат слова $-f_{n_{1}}$, так, чтобы $\left\|F-f_{n_{1}}\right\|<\delta_{1}$ и для элементов $z \in S^{\mathbb{N}}$ из множества $A_{1}$ меры $\mu^{\infty}\left(A_{1}\right)>1-\delta_{1}$ первая координата стабилизировалась: $\widehat{z}_{1}=\widehat{z}_{1}^{n_{1}}$, последнее возможно в силу стабильности. Предположим, что мы построили аппроксимирующие функции $f_{n_{1}}, \ldots, f_{n_{k-1}}$. Следуюшая функция $f_{n_{k}}$ строится сходным образом-по $\delta_{k}$ и $n_{k-1}$ находим $n_{k}$ и $f_{n_{k}}$ так, чтобы норма разности $F$ и $f_{n_{k}}$ была бы менњше $\delta_{k}$ и для элементов $z \in S^{\mathbb{N}}$ из некоторого множества $A_{k}$ меры $\mu^{\infty}\left(A_{k}\right)>1-\delta_{k}$ координаты с номерами $n_{k-1}$ стабилизировались: $\widehat{z}_{i}=\widehat{z}_{i}^{n}, n>n_{k}, i=1, \ldots, n_{k-1}$. Такие $n_{k}$ и $f_{n_{k}}$ сушествуют в силу стабилизации и предыдушей теоремы.

Утверждается, что такая последовательность $f_{n_{k}}$ может стремиться при $k \rightarrow \infty$ разве лишш к константе и, следовательно, $F=$ const.

Далее более удобно говорить о множествах уровня, а не о функциях, или, что тоже самое, - о характеристических функциях. Предположим, что $A$ есть множество уровня функции $F$ меры, отличной от 0 и 1 . По предположению оно должно быть независимьм от всех стабильных функций. С другой стороны, это множество сколь угодно точно аппроксимируется множествами уровня функций $f_{n_{k}}$, а каждая из этих функций, например, $f_{n_{k}}$, по определению зависит лиш от $n_{k}$-стабильных координат $\widehat{z}_{i}^{n}, i<n_{k}$.

Следовательно, совокупность этих двух требований приводит к тому, что функция $F$ и ее множества уровней с произвольной точностью приближаются функциями, зависящими от нестабильных, но $n_{k}$-стабильных координат. Последнее означает, что координаты нормальной формы слов длины $n_{k}$, от которых зависят аппроксимирующие функции и их множества уровней, не являются стабильными (коротко - нестабильны). Таким образом, при каждом $k$ из всех $n_{k}$-стабильных координат, определяюших $n_{k}$-нормальные формы, мы выбираем подмножество (зависящее от $\delta_{k}$ ) $J_{k} \subset\left(1, \ldots, n_{k}\right)$ лиш тех, которые могут в дальнейшем измениться, - в частности, в него не входят координаты с номерами, меньшими $n_{k-1}$. Поэтому наше построение гарантирует, что множества $J_{k}$ при разных $k$ почти не пересекаются, точнее, их возможные малые пересечения оцениваются через параметры $\delta_{k}$. С другой стороны, эти множества уровня должны сходиться к множеству уровня функции $F$. Сравним два таких множества при разных $k$ : возьмем по данному $\delta$ достаточно большие номера $r<s$, тогда соответствуюшие множества уровня должны отличаться по мере не 
больше, чем на $\delta$, а определяются они непересекающимися множествами координат в исходном пространстве и поэтому должны быть почти независимыми (не представляет труда сделать оценки этой независимости в терминах скорости стабилизации). Такое может быть, только если исходное множество уровня имело меру 0 или 1.

Остается заметить, что предположение о независимости функции $F$ несущественно - если разбиение на уровни стабильных функций не совпадает с разбиением $\sigma$, то всегда существует дополнительное разбиение, котороена множестве положительной меры может быть превращено в независимое дополнение введением плотности. Наше рассуждение при этом не меняется. Предположение о независимости вьше играло лишш методическую роль.

ЗАмЕЧАНИЕ 2. В следующем параграфемы приведем описание границы свободной разрешимой группы ступени два и опишем границу некоторых сплетений. В этих случаях не используются нормальные формы, поскольку первьй шаг (нахождение стабильных функций) описывается не в терминах нормальных форм, а с помощью геометрических координат гранишы. Однако метод доказательства полноты границы - в точности тот же, что и вьше, - описания пределов естественного класса стабильных функционалов от траекторий блуждания на свободной разрешимой группы.

По-видимому, можно, несколько детализировав это рассуждение, получить при тех же предположениях утверждение, что и гранища Мартина в случае стабильности нормальной формы есть множество несократимых бесконечных слов в принятом выше смысле. Во всяком случае, доказанная теорема, в частности, повторяет известные результаты о границах Пуассона-Фюрстенберга для свободных [24] и близких к ним групп.

Если нормальная форма стабильна, то граница представляется не только как фактор-пространство, но и в виде подмножества того же пространства последовательностей образуюших $S^{\mathbb{N}}$ со своей мерой - образом гармонической меры. Разумеется, эти две меры взаимно сингулярны. Как уже говорилось, это верно в случае свободных и локально свободных групп (см. далее), фуксовых групп (см. [27]) и всегда, когда в группе есть так назьваемое строго марковское свойство (см. [63]), как в гиперболических группах по Громову. Однако большого разнообразия групп со стабильной нормальной формой пока найти не удалось. Например, неизвестно существует ли для групп кос стабильная нормальная форма. Эксперименты, которые по просьбе автора провел профессор П. Дюрнуа с предложенной им нормальной формой кос, показали, что и она не обладает стабильностью: форма записи случайной косы не стабилизируется со временем, более того, она полностью и многократно обновляется, начиная с первого символа.

Поэтому возникает следующая по сушеству алгебраическая или логическая проблема - в каких сильно әкспоненииальных группах существует система образующих, для которой какая-либо нормальная форма стабильна?

По сообщению профессора Д. Эпштейна для автоматных групп этот вопрос, по-видимому, решается методами теории, развитой в [47].

Но можно пытаться описать границу в координатах, никак не связанных с исходным алфавитом и нормальными формами. Поиски явной записи гранищы групшы сходны в этом смысле с явным описанием пространства орбит действия групп или с заданием классов эквивалентности неявно заданного отношения эквивалентности - термины, 
в которых следует дать ответ, не определены заранее, и их отыскание есть творческая задача. Кроме того, стабильная нормальная форма, даже если она сушествует, может быть неминимальной или громоздкой, и поэтому полезно использовать естественные геометрические координаты вместо "словарных".

Другой вопрос возникает при сравнении систем образующих группы и мер на них: как сравнивать границы одной и той же группы с разньми мерами? Экспериментально можно установить, что для некоторых групп границу, которая по определению зависит от оператора (т.е. от меры), для широкого класса мер можно моделировать на одном и том же топологическом пространстве, но с различными гармоническими мерами. Так дело обстоит, например, со свободными группами и др., где для всех финитных мер граница есть множество бесконечных несократимых слов. Это дает возможность сравнивать исходные меры и системы образующих уже с помощью соответствуюших гармонических мер. Насколько мне известно, вопрос можно считать относительно изученным только для подгрупा $S L(2, \mathbb{Z})$ и свободных групп.

Соберем в заключение вместе все отмеченные выше факты о нетривиальности границ $\Gamma(G, \mu)$.

ТЕОрема 5. Следующие предложения әквивалентны.

1. Граница Г( $\mu$ ) нетривиальна (не состоит из одной точки), иначе говоря, разбиение $\pi(\mu)$ пространства $\left(S^{\mathbb{N}}, \mu^{\infty}\right)$ не равно тривиальному.

2. Стационарная граница нетривиальна, иначе говоря, разбиение $\sigma(\mu)$ пространства $\left(S^{\mathbb{N}}, \mu^{\infty}\right)$ нетривиально. При әтом стационарная граница совпадает с границей.

3. Существуют непостоянные положительные ограниченные гармонические функиии относительно оператора перехода, определяемого мерой $\mu$.

4. Энтропия блуждания положстельна: $h_{\mu} \geqslant 0$.

5. Энтропия полиморфизма границы $T_{\sigma}$ положительна.

6. Математическое ожидание логарифма вероятности слова по мере $\mu^{n}$ растет с линейной скоростью по $n$.

7. Снос положителен: $l_{\mu} \geqslant 0$, т.е. средняя по мерам $\mu^{n}$ длина слов растет линейно.

Доказательства этих фактов, большинство которых известно (см. [8], [10] и имеюшиеся там ссылки), следует из приводившихся рассуждений. Пункт 5 будет разъяснен ниже.

3.3. Полиморфизм границы, его энтропия и фундаментальное неравенство. В этом пункте мы дадим истолкование введенных в предыдущем параграфе асимптотических параметров в терминах гранищы блужданий и опишем более подробно структуры, сушествующие на границах, в частности, определим так назьваемьй полиморфизм гранищы. Мы не будем использовать ту или иную модель границ и дадим интерпретацию введенных численных констант в терминах бернуллиевской модели в инвариантной форме. Значительная часть материала этого пункта посвящена определениям, связанным с понятием полиморфизма, в частности, началам энтропийной теории полиморфизмов.

Граница $\Gamma(G, \mu)$ (для правого случайного блуждания) является левым $G$-прост- 
ранством, действительно, правый сдвиг

$$
R_{g}\left(z_{1}, z_{2}, \ldots\right)=\left(g, z_{1}, z_{2}, \ldots\right),
$$

где $g \in S$, сохраняет инвариантным разбиение $\sigma$. Поэтому он корректно определен на фактор-пространстве по разбиению $\sigma$, т.е. на границе, и задает там действие образуюшей $g$. Сдвиг $R_{g}$ переводит меру $\mu^{\infty}$ в абсолютно непрерывную меру, но не равную, и даже не эквивалентную, мере $\mu^{\infty}$. Группа, натянутая на сдвиги $R_{g}$, задает действие группы $G$ с квазиинвариантной гармонической мерой $\widehat{\mu}$ на границе Г. В обычных терминах марковской цепи, порожденной случайным блужданием, это действие состоит в приписывании слева элемента $g$ всем элементам траектории случайного блуждания, иначе говоря, в сдвиге на $g$ всей траектории. Отметим, что действие групшы $G$ на гранище $(\Gamma, \widehat{\mu})$ как на пространстве с мерой эргодично и аменабельно в смысле Зиммера как действие с квазиинвариантной мерой [64] для любой (даже неаменабельной) группы. Это, в частности, означает (следствие теоремы Конна-Фельдмана-Вейсса [65]), что траекторное разбиение действия групшы - ручное (гиперконечное), т.е. изоморфно траекторному разбиению действия группы $\mathbb{Z} \mathrm{c}$ квазиинвариантной мерой и, следовательно, допускает монотонную аппроксимацию измеримыми разбиениями. Эти факты тесно связаны с тем способом описания действия (траектории полиморфизма границы), который описан ниже.

Действие групшы $G$ на границе с квазиинвариантной гармонической мерой определяет в $L^{2}(\Gamma, \widehat{\mu})$ унитарное представление этой групшы. Спектральная теория этого представления, в частности, элемента $P_{\mu}$, задаюшего блуждание, тесно связана с материалом, излагаемым далее.

Почти каждая точка границы определяет условный марковский процесс, траектории которого сходятся к этой точке границы, соответствующая ему мера сосредоточена на одном элементе разбиения $\sigma$ в пространстве $S^{\mathbb{N}}$. Переходные вероятности этих условных случайных блужданий уже зависят от выбранной точки границы и могут быть легко выражены через исходную меру и значения гармонической функции, отвечающей точке границы, на образующих, но общее свойство всех условных процессов в том, что их копереходные вероятности одинаковы, более того, всякий марковский процес с теми же копереходными вероятностями есть смесь (интеграл) таких процессов. На важность понятия копереходных вероятностей обращал внимание в своих статьях 60-х гг. Е.Б. Дьнкин [66]. Это утверждение вытекает из того, что копереходные вероятности суть в точности значения гармонических функций, т.е., как было отмечено выше, условные вероятности (относительно фиксированной точки гранищы) начал траектории случайного блуждания. Описание мер с заданными копереходными вероятностями аналогично описанию квазиинвариантных (в частном случае, инвариантных) мер относительно некоторого отношения эквивалентности и с заданным коциклом Радона на этом отношении.

Поскольку разбиение $\sigma(G, \mu)$, вообще говоря, не инвариантно относительно левого сдвига $T$ как эндоморфизма Бернулли пространства $\left(S^{\mathbb{N}}, \mu^{\infty}\right)$, то он не индуцирует однозначное преобразование на фактор-пространстве по этому разбиению $S^{\mathbb{N}} / \sigma=\Gamma$. Но он определяет полиморфизм, т.е. многозначное или марковское преобразование фактор-пространства. Отсылая за подробностями определений и обшей теории полиморфизмов к работе автора [28], приведем основные определения в нужной нам форме. 
ОПРЕДЕЛЕНИЕ 9. Полиморфизмом пространства Лебега $(Y, \varphi)$ с инвариантной мерой называется пара: пространство Лебега $(Y \times Y, \psi)$ и диаграмма

$$
(Y, \varphi) \leftarrow(Y \times Y, \psi) \rightarrow(Y, \varphi),
$$

где стрелки означают гомоморфизмы-проекции на первую (левая стрелка) и вторую (правая стрелка) координаты пространства $Y \times Y$, переводяшие меру $\psi$ в меру $\varphi$. Всякий эндоморфизм есть полиморфизм, причем мера $\psi$ на $Y \times Y$ сосредоточена на графике этого эндоморфизма. Класс полиморфизмов $\bmod 0$ определяется обычным образом.

Если $T$ - произвольньй эндоморфизм пространства Лебега $(X, \mu)$, а $\xi$ - произвольное измеримое разбиение, то канонически определен фактор-полиморфизм пространства $(X / \xi, \mu / \xi)$ с инвариантной мерой, где мера $\psi$ на произведении $(X / \xi \times X / \xi)$ определена своими значениями на прямоугольниках следуюшим образом:

$$
\psi(A \times B)=\mu\left(T\left(\pi^{-1} A\right) \cap \pi^{-1} B\right),
$$

здесь $\pi$ - каноническая проекция, $\pi:(X, \mu) \rightarrow(X / \xi, \mu / \xi)$. Если разбиение $\psi$ инвариантно относительно $T$, то фактор-полиморфизм есть mod 0 фактор-эндоморфизм $T / \xi$ пространства $(X / \xi, \mu / \xi)$, а мера $\psi$ сосредоточена на графике фактор-эндоморфизма $T / \xi$. Точно так же определяется фактор-полиморфизм полиморфизма. Будем обозначать фактор-полиморфизм эндоморфизма или полиморфизма $T$ по произвольному разбиению $\xi$ тем же символом, каким обычно обозначаются фактор-преобразования: $T_{\xi}$. Обрашение порядка стрелок в определении полиморфизма задает полиморфизм П ${ }^{*}$, сопряженньй с полиморфизмом П. Для автоморфизма это обратньй автоморфизм, для эндоморфизма это так называемый әкзоморфизм (=один прообраз, но несколько образов).

Неформально, полиморфизм это многозначное преобразование пространства $(Y, \varphi)$, график которого как подмножество квадрата $Y \times Y$ есть носитель меры $\psi$, а образы каждой точки снабжены вероятностями. Можно также сказать, что это марковское преобразование пространства $(Y, \varphi)$, сопоставляющее почти каждой точке $y \in Y$ некоторую меру на $Y$, а именно, условную меру, индуцированную мерой $\psi$ на слое $y \times Y$. Понятие полиморфизма есть метрический аналог понятий бирасслоения, многозначного отображения, соответствия и др. в соответствующих категориях, специфика теории меры в том, что оно определяется не только графиком, как в перечисленных случаях, а мерой на графике. Поскольку условные меры определены почти всюду, то полиморфизм, в отличие от марковского преобразования, определен с точностью до множеств меры нуль, и все связанные с ним объекты, как обычно в теории меры, понимаются как классы $(\bmod 0)$. Важность этого понятия, в частности, в том, что становится возможной факторизация эндо- и автоморфизмов не только по инвариантным, но ипо произвольным разбиениям. Кроме того, аппроксимация полиморфизмами автоморфизмов позволяет упростить некоторые факты энтропийной и аппроксимационной теории.

Функциональньй аналог полиморфизма П есть марковский оператор $U_{\Pi}$, действующий в пространстве $L^{2}(Y, \varphi)$ по формуле:

$$
\left(U_{\Pi} f\right)(y)=\int_{Y} f(x) \mu_{y}(d x)
$$

где $\mu_{y}$ - условная мера на слое $y \times Y$. 
Этот оператор является сжатием (норма равна единице), он положителен (сохраняет конус неотрицательных функций), вещественен (коммутирует с комплексным сопряжением) и сохраняет (как и сопряженньй оператор) единицу. Наоборот, всякий оператор $U$ с этими свойствами соответствует единственному mod 0 полиморфизму ( $\left.U=U_{\Pi}\right)$. Сопряженному полиморфизму $\Pi^{*}$ отвечает в $L^{2}$ марковский оператор $U_{\Pi}^{*}$, сопряженньй с $U_{\Pi}$. Полиморфизмы образуют вьпуклую полугруппу в векторном пространстве операторов, группой обратимых элементов которой является группа автоморфизмов, плотная в полугруппе. Более подробное изложение см. в [28].

Всякий полиморфизм П с инвариантной мерой пространства $(X, \mu)$ однозначно определяет стационарньй марковский процесс с пространством состояний $(X, \mu)$ и мерой на $X \times X$, отвечающей полиморфизму П в качестве двумерного распределения этого процесса. Автоморфизм сдвига в пространстве траекторий процесса обозначим через $V(\Pi)$. Переход от П к $V(\Pi)$ соответствует взятию дилатации (неминимальной) в терминах теории операторов. Отметим, что операция перехода от П к $V(\Pi)$ не коммутирует с переходом к фактор-полиморфизму: если $\Pi_{1}$ есть фактор-полиморфизм $\Pi_{2}$, то $V\left(\Pi_{1}\right)$ не есть фактор-автоморфизм автоморфизма $V\left(\Pi_{2}\right)$.

Полиморфизм назьвается точныцм, если у него нет фактор-эндоморфизмов, и вполне точным, если соответствуюшая марковская цеп - регулярна, т.е. имеет тривиальное пересечение сигма-алгебр прошлого. В случае конечных или счетных цепей эти два понятия совпадают, но для обшего случая они различны (см. [56], [57]).

Простейшие примеры полиморфизмов - конечные выпуклые линейные комбинации эндо- и автоморфизмов с коэффициентами, зависяшими от точки: $\Pi(x)=\sum_{i} p_{i}(x) \cdot T_{i} x$, равенство понимается как равенство условных мер перехода из точки $x$. Если $p_{i}-$ постоянны, то П есть полиморфизм, соответствующий случайному блужданию по траекториям эндоморфизмов $T_{i}$. Если $p_{i}-$ неотрицательные измеримые функции с единичной суммой, а $T_{i}$ - несингулярные преобразования, возможно, даже не эпиморфные, то такой полиморфизм назовем конечно разложимым. Однако такие полиморфизмы не исчерпьвают запас всех полиморфизмов, более того, даже если заменить в формуле сумму на интеграл, мы получим лишь малую часть всех возможностей: сушествуют и образуют всюду плотное $G_{\delta}$ множество экстремальные точки в выпуклом компакте полиморфизмов, отличные от эндоморфизмов (см. [28]).

ОПРЕДЕЛЕНИЕ 10. Полиморфизмом $T_{\sigma}$ границъ $(\Gamma, \widehat{\mu})$ назьвается фактор-полиморфизм левого сдвига $T$ пространства $\left(S^{\mathbb{N}}, \mu^{\infty}\right)$ по (неинвариантному) разбиению $\sigma$.

Из определения полиморфизма границы ясно, что он есть конечная выпуклая комбинация (с, вообще говоря, непостоянными коэффициентами) несингулярных сдвигов границы на элементы образуюших группы $G$.

Рассмотрим простейший нетривиальный пример полиморфизма границы - полиморфизм гранишы свободной группы.

Пусть $F_{d}$ - свободная группа с $d$ образуюшими, $S$ - множество образуюших и обратных к ним, а $\mu$-равномерная мера на $S$. Как было сказано, граница $\Gamma\left(F_{d}, \mu\right)$ есть пространство бесконечных несократимых слов; оно есть фактор-пространство пространства $\left(S^{\mathbb{N}}, \mu^{\infty}\right)$ по разбиению $\sigma$, элементы которого определяются как множества бесконечных слов, преврашаюшихся после сокрашений (сокрашения делаются слева направо) в одно и то же несократимое слово. Это разбиение неинвариантно относительно сдвига, поэтому при факторизации сдвиг $T$ определяет 
не фактор-Эндоморфизм, а следуюший фактор-полиморфизм $\Pi=T_{\sigma}$ гранишы: образ $T_{\sigma}\left(z_{1}, z_{2}, z_{3}, \ldots\right)$ бесконечного несократимого слова $\left(z_{1}, z_{2}, z_{3}, \ldots\right)$ есть с некоторой вероятностью $\alpha$ сдвиг влево этого несократимого слова, т.е. слово $\left(z_{2}, z_{3}, \ldots\right)$, и с вероятностями $\frac{1-\alpha}{2 d-1}-$ правый сдвиг со вставкой новой образующей, т.е. слово $\left\{\left(g, z_{1}, z_{2}, z_{3}, \ldots\right)\right\}$, где $g$ - произвольная образуюшая или обратная к ней, отличные от обратной к $z_{1}$, вероятности всех вставок - одинаковы. Константа $\alpha$ может быть вычислена, она равна вероятности того, что первая буква несократимого слова есть первая буква исходного (сократимого) слова; как будет видно далее, это есть значение гармонической функции на образующих, которое зависит только от первой буквы несократимого слова. Это дает возможность легко вычислить все гармонические функции на свободной групше: значение гармонической функции на элементе $g \in S$, отвечающей несократимому слову $\gamma$ (= точке гранишы), начинаюшемуся с символа $h \in S$, равно $\alpha$, если $g h=e$, и $\frac{1-\alpha}{2 d-1}-$ в противоположном случае. Обратим внимание на то, что в примере со свободной группой определен и обычньй сдвиг на самой границе, сохраняюший меру, поскольку граница есть марковский компакт, состояший из всех бесконечных несократимых слов, т.е. таких, в которых соседние координаты не являются взаимно обратньми. Поднятие этого сдвига в пространство $\left(S^{\mathbb{N}}, \mu^{\infty}\right)$ есть довольно громоздкий оператор - “случайный сдвиг" $T \omega$, где $\omega$ - случайная целочисленная функция. Но все это - специфическая особенность свободной группы, и в общем случае нельзя определить канонического эндоморфизма гранищы. Возможно, такой сдвиг определен и в случаях, когда имеется стабильная нормальная форма (см. выше).

Рассмотрим теперь полиморфизм гранищы $T_{\sigma}$ в случае произвольной сильно экспоненпиальной группы с мерой и свяжем его с действием группы $G$ на границе. Напомним, что траекторией точки $x$ под действием полиморфизма П назьвается множество точек $y$, для которых существуют такие натуральные $n, m$, что образы $\Pi^{n} x$ и $\Pi^{m} y$ имеют непустое пересечение. Разбиение на траектории назьвается траекторным разбиением полиморфизма, оно также определено $(\bmod 0)$. Непосредственно из определения ясно, что

ЛЕмма 7. Траекторное разбиение полиморфизма $T_{\sigma}$ совпадает с траекторным разбиением группь $G$ в смысле определенного выше действия группь на границе. Вероятность перехода из точки границь $\gamma$ в точку $g \gamma$, әде $g \in S$, равна значению гармонической функиии $f_{\gamma}(g)$ в точке $g$, т.е. условной мере того, что траектория блуждания, пришедшая в пределе в точку границь $\gamma \in \Gamma$, стартовала в первый момент времени с әлемента $g \in S$.

В соответствии со сказанным выше полиморфизм $T_{\sigma}$, как и всякий полиморфизм, определяет стационарный марковский процесс; пространством его состояний является граница $\Gamma(G, \mu)$, переходные вероятности полиморфизма $T_{\sigma}$ определяются гармоническими функциями и, вообше говоря, зависят от точки границы. Для построенного полиморфизма в силу его определения гармоническая мера $\widehat{\mu}$ (фактор-мера) является конечной инвариантной мерой. Этот марковский процесс является также случайным блужданием, но уже не на группе $G$, а на ее границе - $\Gamma(G, \mu)$, и блуждание совершается по траекториям действия группы $G$ на границе. Этот процесс можно было бы назвать вторичнымм или граничнымм случайным блужданием. Обозначим сдвиг в 
пространстве траекторий этого процесса через $V\left(T_{\sigma}\right)$. Свойства этого процесса представляют большой интерес: например, будет ли сдвиг $V\left(T_{\sigma}\right)$ изоморфен сдвигу Бернулли? Полиморфизм $T_{\sigma}$ никогда не является эндоморфизмом (т.е. детерминированным преобразованием границы), за исключением случая свободной полугруппы.

Упомянем в этой связи, что в теории случайных блужданий, начиная с работ Фюрстенберга, рассматривают другой марковский оператор на гранище Пуассона - блуждание по образующим группы $G$, действующей на гранище с вероятностями $\mu(\cdot)$, т.е. с постоянными вероятностями перехода, поэтому полиморфизм также есть конечная выпуклая комбинация несингулярных преобразований. Однако полиморфизм границыне совпадает с ним; как мы видели, вероятности перехода, вообще говоря, непостоянны. Гармоническая мера является инвариантной по отношению к обоим полиморфизмам, или, в принятой терминологии, - стационарна по отношению к группе с мерой $(G, \mu)$; траекторные разбиения обоих полиморфизмов совпадают, однако нам кажется, что полиморфизм $T_{\sigma}$ вскрывает более глубокие свойства случайных блужданий. Двусторонний марковский стационарный процесс, порожденньй полиморфизмом $T_{\sigma}$, также имеет естественное истолкование в терминах случайных блужданий, на чем мы здесь не останавливаемся.

Начала общей эргодической теории полиморфизмов изложены в [28] (аппроксимация, связь с марковскими процессами, выпуклая и алгебраическая структуры), что же касается таких фактов, как индивидуальная и статистическая эргодические теоремы, то они были доказаны еше в старых работах Э. Хопфа, Чакона-Орнштейнаи др. (см., например, [67]). Вопрос об энтропии полиморфизмов до сих пор не затрагивался. Определение, предлагаемое ниже, обобшает определение колмогоровской энтропии автоморфизмов и не является вполне традиционным и в этом случае. Грубо говоря, энтропийная теория полиморфизмов сводится $\kappa$ расширению энтропийной теории эндоморфизмов, охватывающему случай неинвариантных разбиений.

Напомним сначала, что полиморфизм конечного пространства $\{1,2, \ldots, n\}$ с инвариантной равномерной мерой задается бистохастической матрицей $\left(p_{i, j}\right)$, и ее энтропия $h$, т.е. энтропия соответствуюшей марковской цепи, определяется как средняя условная энтропия матрищы переходов: $h=\sum_{j} p(j) \cdot H\left(P_{j}\right)$, где $p(j)=\sum_{i} p_{i, j}$, $P_{j}=\left(p_{1, j} / p(j), \ldots, p_{n, j} / p(j)\right)$. Число $h$ мы по определению считаем энтропией конечного полиморфизма, оно же является колмогоровской энтропией соответствующей марковской цепи, т.е. $h=H\left(\xi \mid T^{-1} \xi\right)$, где $\xi$ - марковская образующая, а $T$ - сдвиг в пространстве ее траекторий.

ОПРЕДЕЛЕНИЕ 11. Пусть П - полиморфизм пространства Лебега $(Y, \varphi)$ и $\left\{\zeta_{n}\right\}-$ произвольная последовательность конечных разбиений, стремящаяся (необязательно монотонно) к разбиению на точки пространства $(Y, \varphi)$. Определим энтропию полиморфизма П вдоль данной последовательности конечных разбиений:

$$
h\left(\Pi,\left\{\zeta_{n}\right\}\right)=\limsup _{n} h\left(\Pi_{\zeta_{n}}\right),
$$

и энтропию полиморфизма:

$$
h(\Pi)=\sup h\left(\Pi,\left\{\zeta_{n}\right\}\right),
$$

здесь $\Pi_{\zeta_{n}}-$ конечный фактор-полиморфизм пространства $Y / \zeta_{n}$, а последний супремум берется по всем таким последовательностям конечных разбиений $\left\{\zeta_{n}\right\}$. 
ЛЕмма 8. 1. Энтропия полиморфизма, совпадающего $\bmod 0$ с некоторым эндоморфизмом, равна классической әнтропии этого әндоморфизма.

2. Энтропия полиморфизма, не равного $\bmod 0$ әндоморфизму, всегда положительна, следовательно, она равна нулю, только если полиморфизм есть mоd 0 әндоморфизм с нулевой әнтропией.

ДокАЗАТЕЛЬСтво. 1. Пусть $T$ - эндоморфизм. Поскольку разбиения $\zeta_{n}-$ конечны, то фактор-полиморфизм автоморфизма $T$ есть полиморфизм конечного пространства, т.е. задается бистохастической матрищей (матрицей переходов), и энтропия конечного полиморфизма выгисляется по классической формуле энтропии конечных марковских цепей, приведенной вьше, т.е. по формуле для средней условной энтропии. Поэтому правая часть есть верхний предел средних условных энтропий

$$
H\left(\zeta_{n} \mid T^{-1} \zeta_{n}\right)
$$

по последовательности конечных разбиений, стремяшихся к разбиению на отдельные точки; супремум по всем последовательностям совпадает с энтропией эндоморфизма в силу следующей очевидной формулы, легко выводимой из общих свойств средней условной энтропии (см. [67; леммы 5.9-7.2]):

$$
H\left(\xi^{n} \mid T^{-1} \xi^{n}\right)=H\left(\xi \mid T^{-1} \xi^{n}\right)
$$

действительно, правая часть стремится к $H\left(\xi \mid T^{-1} \xi\right)$, а супремум этих выражений и есть энтропия $h(T)$ в колмогоровском определении. Это оценивает энтропию эндоморфизма сверху нашей энтропией. Противоположная оценка следует из существования счетной образующей эндоморфизма с максимальной энтропией (см. [67]):

$$
h(T)=h(T, \xi)=H\left(\xi \mid T^{-1} \xi\right) .
$$

2. Рассмотрим марковский сдвиг $V(\Pi)$, отвечающий полиморфизму П. Из нашего определения энтропии $h(\Pi)$ ясно, что она больше или равна средней условной энтропии переходных вероятностей марковского сдвига (т.е. полиморфизма):

$$
h(\Pi) \geqslant h\left(\xi \mid V(\Pi)^{-1} \xi\right),
$$

здесь $\xi$ - марковская образующая для $V(\Pi)$. Поэтому если полиморфизм не совпадает с эндоморфизмом, т.е. его переходы не являются детерминированными почти всюду, то и энтропия полиморфизма $h(\Pi)$ положительна. Если же полиморфизм П совпадает $\bmod 0$ с эндоморфизмом $S$, то марковская цеп детерминирована и энтропия $h(\Pi)$, как доказано в п. 1 , равна энтропии $h(S)$.

Пункт 1 леммы показывает, что переход к полиморфизмам полезен и для теории эндоморфизмов - он позволяет расширить теорию аппроксимаций эндоморфизмов, а именно, всякий эндоморфизм очевидньм образом является слабым пределом конечных полиморфизмов, т.е. конечных марковских цепей (см. [28]), и вычисления энтропии упрощаются существенньм образом. Эти упрощения происходят за счет отказа 
от постоянного использования только инвариантных разбиений. Заметим, что некоторое преимушество определения Синая по сравнению с первоначальным определением Колмогорова как раз в том, что в нем не используются полностью свойства инвариантных разбиений. Отличие предлагаемого способа вычислений энтропии от общепринятого еще и в том, что предельный переход совершается не по измельчениям данного разбиения с помощью последовательных сдвигов и последующим взятием супремума по конечным разбиениям, а по произвольной последовательности разбиений. Наше определение применимо также ко всем несингулярным преобразованиям (при этом матрища $\mu\left(T\left(A_{i}\right) \cap A_{j}\right)$ будет уже не бистохастической, а лишш марковской).

Энтропия $h(\Pi)$ полиморфизма П, вообще говоря, не совпадает ни с энтропией соответствующего марковского сдвига $h(V(\Pi))$, ни со средней условной энтропией переходов. Например, для полиморфизма случайного блуждания с равньми вероятностями по траекториям $\left(T, T^{-1}\right)$, где $T$ - автоморфизм с положительной энтропией, $h(\Pi)=2 h(T)$, в то время как $h(V(\Pi))=h(T)$. Точно так же, если полиморфизм есть эндоморфизм, то условная энтропия переходов равна нулю, хотя его энтропия может быть положительной; но вот нетривиальньй пример того же эффекта из теории убываюших последовательностей (см. также Добавление): для блуждания по траекториям бернуллиевского действия свободной группы с двумя образуюшими и вероятностями $(1 / 2,1 / 2)$ - см. [69], [39] - энтропия марковской цепи равна бесконечности, а условная энтропия переходов равна единице. Попутно заметим, что небольшое видоизменение этого примера дает положительньй ответ на старый вопрос, поднятьй в работах В. А. Рохлина и Я. Г. Синая: для автоморфизма Бернулли с бесконечной энтропией можно найти $K$-образуюшую, для которой условная энтропия на один шаг имеет наперед заданное значение.

Таким образом, для полиморфизма П и соответствующего марковского сдвига $V(\Pi)$, порожденного полиморфизмом П, мы определили три, вообще говоря, различные величины:

$$
h\left(\xi \mid V(\Pi)^{-1} \xi\right) \equiv \underline{h}(\Pi)
$$

- среднюю условную энтропию переходных вероятностей полиморфизма (марковского процесса),

$h(\Pi)$

- энтропию полиморфизма, и

$$
h(V(\Pi)) \equiv \bar{h}(\Pi)
$$

- энтропию марковского сдвига. Для эндоморфизмов последние два числа совпадают. В обшем случае имеет место следуюшее неравенство, вытекающее из определений:

$$
\underline{h}(\Pi) \leqslant \bar{h}(\Pi) \leqslant h(\Pi) \text {. }
$$

Проблема вычисления энтропии полиморфизма и энтропии марковского сдвига общего вида (с непрерывным множеством состояний) исследована недостаточно. Заметим, что марковость на самом деле нисколько не уменьшает обшность задачи, поскольку любой $K$-автоморфизм имеет марковскую $K$-образующую - ею может быть любое инвариантное исчерпьвающее (см. [68]) разбиение автоморфизма с вполне положительной энтропией. 
Основной вопрос можно сформулировать так: когда марковская образующая имеет максимальную энтропию, иначе говоря, когда средняя условная энтропия на один шаг равна энтропии марковского сдвига, т.е. $\bar{h}(\Pi)=\underline{h}(\Pi)$, и, в частности, для каких случайных блужданий по траекториям действия групп это так? Ответ, конечно, зависит от свойств группы и характера ее действия. Не исключено, что аменабельность действия играет здесь решаюшую роль. Другой вопрос - когда энтропия марковской цепи равна энтропии полиморфизма, т.е. когда ее можно вьгислять по марковским аппроксимациям, т.е. когда $h(\Pi)=\bar{h}(\Pi) ?$

Формула Абрамова-Рохлина для энтропии косых произведений даже в тех случаях, когда марковский с двиг представляется как косое произведение (случайные блуждания с постоянными переходными вероятностями), здесь не всегда применима, поскольку смешанная энтропия слоев не вычисляется обозримым образом в нужных терминах. C другой стороны, эта формула может быть обобщена на полиморфизмы, при этом она послужит средством выгисления энтропии фактор-полиморфизма, - обычно она применяется для вычисления энтропии накрытия, мы вернемся к этому ниже. Обратим внимание на то, что энтропия полиморфизма не монотонна даже в классе конечных разбиений, т.е. энтропия фактор-полиморфизма может быть больше энтропии исходного полиморфизма (эндоморфизма). Для эндоморфизмов монотонность имеет место, однако условная энтропия $h(T, \xi)$ монотонна лишш в классе разбиений с конечной энтропией и не выполнена для произвольных разбиений. В целом энтропийная теория полиморфизмов должна в сушественном отличаться от известной теории.

В дальнейшем мы ограничимся лишь фактор-полиморфизмами әндоморфизма Бернулли, оставив изучение обшего случая до другого раза. Назовем такие полиморфизмы бернуллиевскими. Полиморфизм границы по определению является бернуллиевским.

Сформулируем теорему об энтропии бернуллиевского полиморфизма гранишы $T_{\sigma}$.

ТЕОРема 6. Энтропия полиморфизма $T_{\sigma}$ границы $(\Gamma(G, \mu), \widehat{\mu})$ равна әнтропии случайного блуждания пары $(G, \mu)$ :

$$
h\left(T_{\sigma}\right)=h_{\mu} .
$$

ДоказАтельство. Рассмотрим $T_{\sigma}$ - полиморфизм границы $(\Gamma(G, \mu), \widehat{\mu})$, т.е. фактор-полиморфизм левого сдвига $T$ пространства $\left(S^{\mathbb{N}}, \mu^{\infty}\right)$ по разбиению $\sigma$. Все рассмотрения будут проводиться в накрывающем пространстве $\left(S^{\mathbb{N}}, \mu^{\infty}\right)$.

Для вычисления энтропии фактор-полиморфизмов $h\left(T_{\sigma}\right)$ согласно нашему определению нужно взять последовательность каких-либо конечных разбиений $\left\{\psi_{n}\right\}$, сходящуюся к разбиению на точки $\bmod 0$ в пространстве $(\Gamma, \widehat{\mu})$, и вычислить $\lim _{n \rightarrow \infty} H\left(\psi_{n} \mid T^{-1} \psi_{n}\right)$. В накрывающем пространстве $\left(S^{\mathbb{N}}, \mu^{\infty}\right)$ задача вычисления $h\left(T_{\sigma}\right)$ сводится к вычислению некоторой поправки энтропии сдвига Бернулли $T$. При этом поскольку в пространстве $\left(S^{\mathbb{N}}, \mu^{\infty}\right)$ последовательность конечных разбиений $\left\{\zeta_{n}\right\}$ (см. п. 3.1) сходится при $n \rightarrow \infty$ к разбиению $\sigma$, то эта поправка есть в пределе условная энтропия $h(T, \sigma)$. Таким образом, вычисления сводятся к следующему:

$$
h\left(T_{\sigma}\right)=H\left(\varepsilon \mid T^{-1} \varepsilon\right)-\lim _{n \rightarrow \infty} H\left(\xi_{n} \mid T^{-1} \xi_{n}\right)=h(T)-H\left(\sigma \mid T^{-1} \sigma\right),
$$


здесь $\varepsilon-$ разбиение mod 0 на точки в том же пространстве, $h(T)$ - энтропия сдвига, равная $H(\mu)$ и равная условной энтропии $H\left(\varepsilon \mid T^{-1} \varepsilon\right)=H\left(\xi_{1} \mid T^{-1} \varepsilon\right)$. Кроме того, легко видеть также, что $\zeta_{1}=\xi_{1}^{0}$ и что $H\left(\sigma \mid T^{-1} \sigma\right)=H\left(\xi_{1}^{0} \mid \sigma\right)$. Следовательно, мы должны доказать, что правая часть равенства

$$
h\left(T_{\sigma}\right)=H\left(\zeta_{1} \mid T^{-1} \varepsilon\right)-H\left(\zeta_{1} \mid T^{-1} \sigma\right)
$$

есть

$$
h\left(T_{\sigma}\right)=h_{\mu} .
$$

Это вычисление уже было проделано в [8] в связи с изучением энтропии случайного блуждания и энтропийного критерия тривиальности границы (понятие полиморфизма границы там не вводилось). Точнее, доказанная в [8] формула в наших обозначениях выглядит так:

$$
H\left(\zeta_{1} \mid \sigma\right)=H(\mu)-h_{\mu}=h(T)-h_{\mu},
$$

или

$$
h(T)-H\left(\zeta_{1} \mid \sigma\right)=h_{\mu}=h\left(T_{\sigma}\right) .
$$

Это равносильно формуле

$$
H\left(\zeta_{1} \mid T^{-1} \varepsilon\right)-H\left(\zeta_{1} \mid T^{-1} \sigma\right)=\lim _{n \rightarrow \infty} \frac{H\left(\zeta_{n}\right)}{n} .
$$

Можно использовать иной по сравнению с [8] вьвод, основанный на следующей аналогии. Если бы вместо $\sigma$ мы имели дело с инвариантным относительно $T$ разбиением и если бы существовало независимое инвариантное дополнение к $\sigma$, например, разбиение $\psi$, то мы бы воспользовались основной формулой энтропийной теории

$$
\lim _{n \rightarrow \infty} \frac{H\left(\psi^{n}\right)}{n}=h(T, \psi) \equiv H\left(\psi \mid T^{-1} \psi\right)
$$

(см. формулы 7.2-7.4 в [68]), где $\psi^{n}=\bigvee_{k=0}^{n-1} T^{-k} \psi$. Подобную формулу можно оправдать и в нашем случае, используя полуинвариантность разбиения $\sigma$. А именно, разбиения $\zeta_{n}$ играют роль $\psi^{n}$, т.е. роль разбиений, аппроксимируюших разбиение на точки в $(\Gamma(G, \mu), \widehat{\mu})$.

Следующая вькладка, аналогичная упомянутой вьше лемме об энтропии эндоморфизма в [68], дает еше одно неравенство для энтропий. Так как имеет место включение $\zeta_{n} \prec T^{-1} \zeta_{n-1} \vee \zeta_{1}$, из которого, собственно, и следует полуинвариантность $\sigma$ (в терминах предыдушего пункта $\left.\xi=\sigma, \eta_{1}=\zeta_{1}\right)$, то

$$
H\left(\zeta_{n}\right) \leqslant H\left(\zeta_{n-1} \vee T^{-1} \zeta_{1}\right)=H\left(\zeta_{n-1}\right)+H\left(\zeta_{1} \mid T^{-1} \zeta_{n-1}\right) .
$$

Применяя обратную индукцию по $n, n-1, \ldots, 1$ и складывая все неравенства, получим

$$
H\left(\zeta_{n}\right) \leqslant \sum_{k=0}^{n-1} H\left(\zeta_{1} \mid T^{-1} \zeta_{k}\right)+H\left(\zeta_{1}\right)
$$

деля на $n$, а затем устремляя $n$ к бесконечности и используя выражение $h_{\mu}$ через $\zeta_{n}$, получим:

$$
h_{\mu} \leqslant H\left(\sigma \mid T^{-1} \sigma\right) \text {. }
$$


ЗАмечАниЕ 3. 1. Формула

$$
h(T)-H\left(\zeta_{1} \mid \sigma\right)=h_{\mu}
$$

может рассматриваться как аналог формулы энтропии косых произведений - формулы Абрамова-Рохлина - для энтропии полиморфизма гранищы, иначе говоря, выражение $H\left(\zeta_{1} \mid \sigma\right)$ имеет смысл "смешанной энтропии слоев". Напомним также, что это выражение есть средняя по всем гармоническим функциям (относительно гармонической меры) энтропия распределения гармонической функции на образуюших.

2. Полным аналогом теоремы Колмогорова-Рохлина-Синая для полиморфизмов в терминах накрываюшего эндоморфизма была бы формула

$$
H\left(\alpha \mid T^{-1} \alpha\right)=\lim _{n \rightarrow \infty} \frac{H\left(\beta_{n}\right)}{n},
$$

где $T$ - произвольный эндоморфизм, $\alpha$ - произвольное (не инвариантное) измеримое разбиение, а $\beta_{n}$ - его базис. Такая (пока не доказанная) формула, точнее, супремум обеих частей этой формулы по некоторому классу последовательностей разбиений давали бы энтропию полиморфизма $T_{\alpha}$. Мы доказали весьма специальный случай этой формулы (для бернуллиевских полиморфизмов).

В конще этого пункта мы применим тот же прием, что и вьше, для более простой задачи - вычисления топологической энтропии.

Прокомментируем теперь с новой точки зрения энтропийный критерий тривиальности границы. Он следует из приведенной теоремы и из известного свойства полной положительности автоморфизмов Бернулли (см. [68]).

ТеОРема 7. Граница Г $(G, \mu)$ тривиальна тогда и только тогда, когда әнтропия $h_{\mu}$ равна нулю.

Этот критерий ( “тогда") доказан впервые в [8] с помощью приведенной вьше формулы; то очевидное обстоятельство, что в случае тривиальной границы энтропия равна нулю, отмечено в первой статье по энтропии блужданий [1]. Фактически, оценки в [8], приводящие к нужной формуле, копируют доказательство факта о полной положительности автоморфизма Бернулли. Аналогия с полной положительностью вскрывает истинную суть энтропийного критерия тривиальности гранищы.

ДокАЗАТЕльство. Если гранища тривиальна (одноточечна), то тривиален и полиморфизм границы и энтропия его равна нулю. Если энтропия $h_{\mu}$ равна нулю, то полиморфизм гранипы есть эндоморфизм mod 0 с нулевой энтропией, и, следовательно, $T_{\sigma}$ есть фактор-эндоморфизм эндоморфизма Бернулли с нулевой энтропией, а тогда он тривиален в силу полной положительности эндоморфизма Бернулли.

Мы видим, что классическое свойство полной положительности энтропии автоморфизма Бернулли (см. [68]) можно очевидным образом обобщить: всякий нетривиальньй (т.е. действующий на неодноточечном пространстве) фактор-полиморфизм эндоморфизма Бернулли имеет положительную энтропию. То же самое относится к любому эндоморфизму с вполне положительной энтропией. 
Обратимся теперь к топологической энтропии полиморфизмов и полиморфизма границы, в частности. Определение топологической энтропии для общего полиморфизма, по-видимому, можно свести к случаю эндоморфизмов таким же образом, что и вьше, - переходом к марковскому сдвигу, но мы не будем рассматривать общий случай, а ограничимся ситуацией, когда полиморфизм возникает как фактор-отображение эндоморфизма компакта, и потому вопрос по определению сводится к подсчету топологической энтропии эндоморфизма в накрывающем пространстве, но в неотделимой топологии. В данном случае этим компактом является $S^{\mathbb{N}}$, а преобразованием - левьй сдвиг $T$. Фактор-отображение берется по разбиению $\sigma$. Заметим, что можно определить и фактор-топологию гранишы с помошью множества гармонических функций, но проще рассмотреть само пространство $S^{\mathbb{N}}$. Топологическую энтропию полиморфизма гранишы мы вычисляем как топологическую әнтропию левого сдвига в $S^{\mathbb{N}}$ относительно (неотделимой) топологии, в которой отождествляются әквивалентные бесконечные слова. В этом случае топологическую энтропию сдвига относительно квазикомпактной топологии можно определить, как обычно (см., например, [58], [70]), а именно, нужно найти минимальное конечное подпокрытие произведения сдвигов данного открытого покрытия и затем взять супремум предела нормированных логарифмов мошности этих подпокрытий по всем измельчаюшимся покрытиям (см., например, [70]).

В нашем случае мы должны проделать это со стандартным левым сдвигом в $S^{\mathbb{N}}$, но при подсчете числа элементов подпокрытий должны принимать в расчет отождествление слов, т.е. факторизацию цилиндров по разбиению $\sigma$. Поскольку разбиение $\sigma$ есть предел цилиндрических разбиений $\zeta_{n}$, то вопрос сводится к подсчету мошности минимального подпокрытия элементов этих конечных разбиений. Буквальная реализация этой схемы приводит к следуюшей вькладке. В силу соотношения $\sigma=$ $\lim _{n \rightarrow \infty} \zeta_{n}$ факторизация цилиндров совпадает с факторизацией по разбиениям $\zeta_{n}$, а число элементов разбиения $\zeta_{n}$ есть число слов длины $n$, поэтому оценка топологической энтропии такова:

$$
h_{\mathrm{top}} \leqslant \lim _{n \rightarrow \infty} \frac{\ln \# \zeta_{n}}{n}=v,
$$

что дает лишь неравенство $h_{\mu}=h\left(T_{\sigma}\right) \leqslant \lim _{n \rightarrow \infty} h_{\text {top }}\left(T_{\sigma}\right)=v_{S}$.

Однако мы можем поступить более аккуратно и получить уже в точности фундаментальное неравенство. Для этого мы выберем подпокрытие более экономно, учитывая, что на множестве полной $\mu^{\infty}$-меры в $S^{\mathbb{N}}$ верно следуюшее.

Для всякого $\varepsilon>0$ существует такое $n$, что цилиндрами минимальной длины $(l+\varepsilon) n$ можно покрыть подмножество в $S^{\mathbb{N}}$, мера которого больше чем $1-\varepsilon$, здесь $l$ - снос; это есть прямое следствие теоремы о сносе. Поэтому можно выбрать последовательность $\varepsilon_{n}$, стремящуюся к нулю, так, что покрытие множества полной меры будет осуществляться элементами разбиений $\zeta_{\left(l+\varepsilon_{n}\right) n}$, где $\varepsilon_{n} \rightarrow 0$. Таким образом, мошность подпокрытий множества полной меры (но не всего $S^{\mathbb{N}}$ !) асимптотически равна числу слов длины $l \cdot n$, где $l-$ снос. Назовем топологию, индуцированную на множестве полной меры обычной топологией на $S^{\mathbb{N}}$, уточненной топологией. Тем самым, топологическая энтропия уточненной топологии оценивается сверху пределом

$$
\widetilde{h}_{\mathrm{top}} \leqslant \lim _{n \rightarrow \infty} \frac{\ln \# \zeta_{l \cdot n}}{n}=l \cdot v,
$$


здесь волна означает переход к уточненной топологии в фактор-пространстве. Нетрудно видеть, что эту оценку нельзя улучшить, не меняя топологию.

Суммируя все предыдущее, получаем следующее утверждение.

ТЕОРема 8. Топологическая энтропия полиморфизма границы относительно уточненной топологии совпадает с произведением логарифмического обтема на снос:

$$
\widetilde{h}_{\mathrm{top}}\left(T_{\sigma}\right)=v_{S} \cdot l_{\mu}
$$

и фундаментальное неравенство есть неравенство мехду топологической $u$ метрической энтропиями для полиморфизма границы:

$$
h_{\mu}=h_{\mu}\left(T_{\sigma}\right) \leqslant \widetilde{h}_{\mathrm{top}}\left(T_{\sigma}\right)=v \cdot l_{\mu} .
$$

ЗАмЕчАНИЕ 4. То обстоятельство, что в определении топологии и, следовательно, топологической энтропии участвует снос, зависяший от меры $\mu$, не должно удивлять, например, топологическую энтропию марковской цепи также определяют с помощью топологической марковской цепи, которая задается матрищей из нулей и единиц, построенной по матрище переходов марковской цепи, а не в полном (бернуллиевском) компакте. Ровно такая же ситуация и здесь: топологическая энтропия определяется не в полном прообразе - компакте $S^{\mathbb{N}}$ всех последовательностей, - тогда бы она была равна $v$, как при первом подсчете, а на меньшем подмножестве, которое учитывает снос.

Здесь уместно обратить внимание на общую задачу об определении естественной компактной (или сигма-компактной) топологии, в которой следует рассматривать данную динамическую систему. А именно, в какой топологии метрическая энтропия данного автоморфизма есть его топологическая энтропия? Считается, что известная теорема Джуитта-Кригера [71], [72] решает этот вопрос: всякий эргодический автоморфизм может быть реализован как гомеоморфизм строго эргодического компакта в этом случае топологическая и метрическая энтропии тривиальным образом совпадают в силу единственности инвариантной меры. Однако это решение неудовлетворительно, поскольку компакт строится искусственно, отдельно от исходного пространства с мерой, в котором задан автоморфизм. На самом деле гораздо ближе к этой задаче находится работа Р. Боуэна [70], в которой сначала определена топологическая энтропия для некомпактных пространств, а затем построено множество полной меры в исходном пространстве, на котором топология (как правило, некомпактная) вводится так, чтобы топологическая и метрическая энтропии эргодического автоморфизма - совпадали; по поводу топологической энтропии в некомпактных пространствах см. также [73]. Остается открытым вопрос, когда можно построить компактную топологию. Кроме того - и это в точности тот вопрос, который обсуждался выше в связи с полиморфизмом гранищы, - можно не настаивать на том, чтобы топологическая энтропия была обязательно равна метрической, но взамен требовать более естественной связи топологической и метрической структур. Именно так мы и поступили - фундаментальное неравенство может оставаться строгим, но уточненная топология определена так, что топологическая энтропия более полно отражает ситуацию (в данном случае топологическая энтропия явно учитьвает снос). В добавлении к этой статье мы также затрагиваем вопрос о множестве топологий, согласованных со структурой данного пространства с мерой, правда, в связи с другими задачами. 
Таким образом, мы привели динамическую интерпретацию фундаментального неравенства. Интересно было бы дать прямое доказательство неравенства для общих полиморфизмов.

Но что же такое мера с максимальной энтропией, если неравенство - строгое? Обычно мерой с максимальной энтропией в контексте теории границ Пуассона-Фюрстенберга называют гиббсовскую меру, определение которой никак непосредственно не связано со случайным блужданием, а связано лишь с действием группы.

В предлагаемом подходе такой мерой следует назьвать меру на гранище, которая является мерой с максимальной энтропией для полиморфизма границь. Но это еше само по себе не проясняет ее связей с исходньм блужданием. Оказывается, в случае строгого неравенства эта мера, если она сушествует, порождается обобщенныцм случайныцм блужданием ("блужданием со вставками"), о чем мы кратко скажем ниже в связи с конкретным примером локально свободной группы (§5). Общее определение будет рассмотрено особо; оно открывает новую связь между групповыми и метрическими конструкциями. Если фундаментальное неравенство есть равенство, то гармоническая мера есть мера максимальной энтропии относительно полиморфизма гранипы. Это имеет место для свободноподобных групा. В общем же случае при новом подходе мера с максимальной энтропией является обобщенной гармонической мерой.

\section{§4. Свободные разрешимые группы}

4.1. Топологическая модель свободной разрешимой группы ступени два. По ряду причин интересно рассмотреть указанныепроблемы для свободных разрешимых групп. Эти групाы подробно изучались алгебраистами в 50-60-х гг. и ранее (в работах Холла, Магнуса, и др. [74]-[76]), позже рассматривался рост нижнего центрального ряда [77], коэффициенты Голода-Шафаревича (см., например, [78]), однако лишь сейчас эти групшы стали предметом исследования с точки зрения гармонического анализа и асимптотических характеристик.

Свободные разрешимые групшы ступени два и вьше не имеют точных матричных представлений и вообше являются в некотором смысле "бесконечномерными" группами, как мы увидим далее. В этом параграфе мы приводим новую топологическую модель для свободных метаабелевых групп, т.е. свободных разрешимых групш ступени два, и вычисляем их границу. Заметим, что наша реализация отличается от известных (базисы в коммутанте Нильсена-Шрайера) инвариантностью построения модели.

Объекты, очень близкие к свободньм разрешимьм группам, - сплетения - давно рассматривались в теории роста и случайных блужданий как естественный источник примеров и контрпримеров. В [29] сплетение $\mathbb{Z} \imath \mathbb{Z}$ использовано как пример группы со сверхэкспоненциальным ростом множеств Фельнера, а в $[8] \mathbb{Z}^{d} \imath \mathbb{Z}, d>2,-$ как пример разрешимой и, следовательно, аменабельной группы с нетривиальной границей. Термин "сплетение" в этих работах не использовался, но привились предложенные тогда термины "лампочные группы" (lamplighter group) и "группа динамических конфигураций”. Сплетения $\mathbb{Z}^{d} \imath \mathbb{Z}$ при $d>2$ дают тем самым важньй пример групा, являюшихся аменабельными группами, но имеющими положительную энтропию. Это явилось неожиданностью - до этого считалось, что для аменабельных групп энтро- 
пия всегда равна нулю [1]. Граница же в этом примере не была вычислена полностью - был дан естественный кандидат - более того, в этом и состояло (в силу критерия) доказательство положительности энтропии, явно она также не выгислена до сих пор. Недавно сплетения использованы также студенткой А. Г. Дюбиной для построения примера квазиизометричных групп, одна из которых разрешима, а вторая не виртуально разрешима, и для примера промежуточного роста сноса.

Теперь становится ясным, что свободные разрешимые группы являются гораздо более естественным и важным классом примеров, чем сплетения с решетками, и те эффекты, которые были открыты в сплетениях, еще более выпукло проявляются в свободных разрешимых группах.

Мы опишем границу этих групп (ступени 2) и, используя тот же метод и переход к свободной разрешимой группе, решим старую задачу о границе сплетений, поставленную в [8].

Начнем с новой, насколько нам известно, модели свободной разрешимой группы ступени 2 , имеющей топологическое истолкование.

Пусть $S o l_{d}^{2} \equiv S o l_{d}-$ свободная разрешимая группа ступени 2 с $d$ образуюшими, т.е. универсальньй объект многообразия разрешимых групп ступени 2 с $d$ образующими. Эту группу можно определить более конструктивно как фактор-группу свободной группы с $d$ образующими по второму коммутанту.

Ее коммутант есть абелева группа с бесконечным числом образующих, которые можно занумеровать элементами $d$-мерной решетки, а именно, - это образы коммутаторов $\left[x_{i}, x_{j}\right], i, j=1, \ldots, r, i \neq j$, исходных образуюших под действием внутренних автоморфизмов, определяемых мономами $x_{1}^{k_{1}} \cdot x_{2}^{k_{2}} \cdots x_{r}^{k_{r}}$ (порядок сомножителей несушественен, так как такой автоморфизм коммутанта, как нетрудно проверить, определяется лишш степенями переменных $k_{1}, k_{2}, \ldots, k_{d}$ и не зависит от их порядка). Сама группа $S o l_{d}$ изоморфна расширению коммутанта с помощью группы $\mathbb{Z}^{d}$, действующей на коммутанте, и некоторого 2-коцикла. (Автор придерживается терминологии, по которой расширяюшей является та группа, которая действует автоморфизмами, а не та, на которой производится это действие, как это принято в алгебре.)

Для $d=2$ коммутант полностью определен, так как между описанными образующими коммутанта нет никаких соотношений и они являются свободными образующими абелевой группы, но при $d \geqslant 3$ описание становится более громоздким, так как между коммутаторами появляются соотношения. Это легко увидеть: например, элемент

$$
x_{1} x_{2} x_{3} x_{1}^{-1} x_{2}^{-1} x_{3}^{-1}
$$

допускает две различных записи через попарные коммутаторы образующих группы и сопряженные с ними элементы, т.е. через выписанные вьше образуюшие коммутанта. Легко понять, что написанньй элемент представляет собой цикл на одномерном остове трехмерного куба и его можно разложить в произведение циклов плоских граней двумя способами. Поэтому использование этих образующих неудобно. В классических работах ([74] и др.) доказьвалось вложение этой групшы в сплетение $\mathbb{Z}^{d} \mathbb{Z}^{d}$, но и оно плохо приспособлено для наших целей, поскольку образ этих групп при вложении в сплетение описывается не столь просто. В готовящейся работе автора и С. В. Добрынина эти вопросы будут рассмотрены подробнее. 
Однако есть прямой способ описания коммутанта и всей группы, отчасти заимствованный из аналогий с теорией кубических гомологий [70]. Он же позволяет легко описать коцикл, задающий расширение.

Будем понимать решетку как одномерный комплекс - топологическое пространство, а именно, как объединение всех сдвигов координатных осей на целочисленные векторы. Чтобы отличить ее от решетки как дискретной группы, обозначим этот одномерньй комплекс через $E^{d}$. Рассмотрим аддитивную группу $\mathbf{B}_{d}$ ориентированных замкнутых 1-циклов на решетке $E^{d}$ как на одномерном комплексе. Ее образуюшими являются әлементарные ииклы (плакеты по физической терминологии или стандартные 1-ииклы в смысле теории кубических гомологий [70]), т.е. циклы, обходящие один раз квадрат решетки в одном из двух направлений. Обход квадрата с вершинами $\left(0, e_{i}, e_{i}+e_{j}, e_{j}\right)$ в указанном порядке, где $e_{i}, e_{j}$ - координатные орты, назовем әлементарнымм $(i, j)$-координатныц ииклом. На группе циклов $\mathbf{B}_{d}$ естественно действует сдвигами группа $\mathbb{Z}^{d}$. Всякий элементарньй цикл есть трансляция одного из элементарных координатных циклов.

Определим 2-коцикл $\beta(\cdot, \cdot)$ группы $\mathbb{Z}^{d}$ со значениями в группе циклов $\mathbf{B}_{d}$. Точнее, мы сразу определяем класс когомологий коциклов. Для этого каждому элементу $v \in \mathbb{Z}^{d}$ решетки сопоставим произвольный связньй путь $\tau_{v}$, соединяюший нуль с элементом $v$ ("путь", как и "решетка", понимается буквально: путь есть непрерьвное отображение полупрямой $\mathbb{R}_{+}$или отрезка в решетку как в топологическое пространство, переводящее целые точки $\mathbb{N} \in \mathbb{R}_{+}$в целочисленные векторы и линейное на каждом целочисленном отрезке). Тогда для пары $(v, w)$ элементов решетки $\mathbb{Z}^{d}$ определяется иикл $\beta(v, w) \in \mathbf{B}_{d}$, составленньй из трех путей:

$$
\left(\tau_{v}, v+\tau_{w},-\tau_{v+w}\right) .
$$

Этот цикл, рассматриваемый как элемент $\mathbf{B}_{d}$, и есть значение 2 -коцикла $\beta(v, w)$.

Лемма 9. Различные выборы системы путей $v \mapsto \tau_{v}$ приводят $к$ когомологичным коциклам.

ДокАЗАТЕльСтво. Действительно, если $v \mapsto \tau_{v}$ и $v \mapsto \rho_{v}$ - две такие системы, то цикл $v \mapsto \tau_{v} \cdot\left(v \mapsto \rho_{v}\right)^{-1}$ есть пленка, осушествляюшая гомологичность.

Обозначим класс гомологий построенного коцикла через $\bar{\beta}$. Обратим внимание на то, что по самому построению этот коцикл тривиален в группе путей, однако он нетривиален как коцикл в групше циклов.

Tеорема 9. Расширение группь ииклов $\mathbf{B}_{d}$ при помощи группы сдвигов $\mathbb{Z}^{d}$ и класса когомологий $\bar{\beta}$ канонически изоморфно свободной разрешимой группе cтупени 2 c d образующими $\mathrm{Sol}_{d}$. При этом изоморфизме образ группв циклов $\mathbf{B}_{d}$ есть в точности коммутант группь $S_{0}$, причем образ әлементарного $(i, j)$-координатного цикла есть коммутатор образующих $\left[x_{i}, x_{j}\right]$, а действие $\mathbb{Z}^{d}$ на циклах переходит в действие $\mathbb{Z}^{d}$ внутренними автоморфизмами на коммутанте группы Sol $_{d}$

Нетрудно доказать, что $H^{2}\left(\mathbb{Z}^{r} ; \mathbf{B}_{r}\right)=\mathbb{Z}$, и построенный коцикл есть образующая этой группы. 
Эта конструкция допускает далекие обобшения. Например, становится понятным, как понимать континуальньй аналог свободных разрешимых групп второй ступени - в них группа циклов на решетке заменяется на аддитивную группу тех или иных 1-форм на $\mathbb{R}^{d}$ (или потоков по Де Раму), а действие $\mathbb{R}^{d}$ и коцикл определяются точно так же, как и выше. Такое обобшение делает понятным, почему свободную разрешимую группу следует считать бесконечномерной, о чем уже упоминалось вьше. Более того, тот же прием можно применить для построения свободных разрешимых групп следуюших ступеней - например, группа ступени 3 может быть представлена тем же способом, так, ее коммутант есть свободная разрешимая группа с бесконечным числом образующих, поэтому второй коммутант естественно представлять как группу 2-циклов на решетке и т. д.

4.2. Граница свободных разрешимых групп и сплетений. Изложим некоторый общий и простой прием, который сводит проблему слов в конечно порож денных группах к комбинаторной геометрии на решетке. Мы увидим, что проблема равенства слов имеет ярко выраженньй геометрический характер: равенство слов в группе равносильно зависящему от группы понятию эквивалентности путей на решетке, отыскание нормальной формы - решению изопериметрической задачи и т. д. По сушеству мы заменяем пространство путей на графе Кэли канонически изоморфным ему пространством путей на решетке. В ряде случаев таких, как изучаемьй далее, этот прием оказывается весьма эффективньм.

Пусть задана группа $G$ с выделенной системой $S=S^{-1}$ из $d$ образующих. Всякий элемент группы можно записать словом в алфавите $S$, а этому слову поставить в соответствие связньй путь на решетке $\mathbb{Z}^{d}$ с началом в нуле следуюшим образом. Отождествим $i$-ю образуюшую с $i$-м координатньм ортом фиксированного базиса решетки $i=1, \ldots, d$. Припишем теперь каждому ориентированному ребру решетки образующую или обратную к ней, которая отвечает координатной прямой, параллельной ребру, беря саму образующую, если ребро проходится в направлении возрастания расстояния от нуля, и обратную к ней, если направление противоположно.

Таким образом, каждое слово есть путь на решетке: пустому слову (единище группы) соответствует путь, состояший из одной нулевой точки решетки, - будем называть его тривиальным; если к уже имеюшемуся слову приписывается некоторая образующая (или обратная к ней), то к концу уже построенного пути присоединяется очередное ориентированное ребро, соответствующее этой образующей или обратной к ней, в зависимости от ориентации.

ОпРЕДЕЛЕНИЕ 12 . Два пути $G$-эквивалентны, если они определяют один и тот же элемент групшы $G$.

Тем самым, проблема тождества слов переформулирутеся как проблема $G$-эквивалентности путей на решетке.

Если соотношения между образуюшими в группе порождаются элементами коммутанта свободной группы, то достаточно определить эквивалентность замкнутого пути (= цикла) тривиальному пути (= циклу). Именно так дело обстоит в нескольких из нижеперечисленных случаев. Но иногда (группа Гейзенберга) незамкнутый путь может быть эквивалентньм замкнутому. В этом случае задание эквивалентности замкнутых путей тривиальному - недостаточно. 
Пример 1. Если $G=\mathbb{Z}^{d}$, то два пути $G$-эквивалентны, если их коншы совпадают. Группа отождествляется с решеткой. Любой замкнутьй путь (заканчивающийся в нуле) эквивалентен тривиальному и определяет единичный элемент группы.

ПримеР 2. Еще один тривиальный пример дает свободная группа. В этом случае два пути эквивалентны, если они совпадут после последовательного сокращения всех соседних ребер (в каждом из путей), которые отличаются лишь ориентацией.

Пример 3. Свободные нильпотентные группь ступени 2. Менее тривиальный пример. Пусть $G$ - свободная нильпотентная группа ступени 2 с $d$ образующими. Сначала рассмотрим случай $d=2$ - это дискретная группа Гейзенберга, т.е. группа целочисленных верхнетреугольных матриц третьего порядка с единицами на главной диагонали. Если образующие суть $a$ и $b$, то соотношения таковы:

$$
[a, b] a=a[a, b], \quad[a, b] b=b[a, b], \quad[a, b]=a b a^{-1} b^{-1}
$$

В этом случае замкнутьй путь эквивалентен нулевому пути тогда и только тогда, когда алгебраическая (ориентированная) площадь, ограниченная им, равна нулю. А два пути $\gamma_{1}$ и $\gamma_{2}$ эквивалентны, если замкнутый путь, образованный $\gamma_{1} \gamma_{2}^{-1}$, эквивалентен тривиальному пути.

Тот же вывод верен и для непрерывной группы Гейзенберга - площадь есть в точности значение симплектической 2-формы (определяющей групп) на соответствующем 2-цикле. Это соображение давно известно и используется в симплектической и неголономной геометрии и теории управления.

При $d>2$ эквивалентность замкнутого пути нулевому описывается тем же критерием, но примененным ко всем проекциям замкнутого пути на двумерные координатные подпространства решетки.

Следующий пример, ключевой для дальнейшего, насколько известно, новьй.

Пример 4. Свободные разрешимые группы ступени 2 . Пусть $G=S_{o l}$. Соотношения между образующими по определению лежат во втором (и, тем более, первом) коммутанте свободной группы. Поэтому достаточно определить эквивалентность замкнутых циклов тривиальному циклу. Но мы даем условие эквивалентности для произвольных путей.

Пусть задан путь $\gamma$; ребро решетки $\rho$ может входить в $\gamma$ с различной ориентацией,+- ; обозначим алгебраическую сумму (с учетом знака) всех вхождений этого ребра в путь $\gamma$ через $\gamma(\rho)$.

ЛЕмма 10. Два конечных пути $\gamma_{1}, \gamma_{2}$ на решетке $\mathbb{Z}^{d}$ являются Sol $_{d}$-эквивалентными тогда и только тогда, когда для всякого ребра $\rho \gamma_{1}(\rho)=\gamma_{2}(\rho)$.

Иначе говоря, эквивалентность означает одинаковость кратностей прохождения (с учетом направлений) ребер решетки вне зависимости от порядка их прохождения; напомним, что в примере 2 (свободная группа) сокрашались лишь последовательно проходимые ребра разной ориентации.

ДокАЗАТЕльство. Доказательство непосредственно вытекает из описания коммутанта группы $S o l_{d}$; действительно, поскольку все коммутаторы групшы $S o l_{d}$ коммутируют, то это значит, что два пути, отличающиеся порядком обхода входящих в них 
плакетов, эквивалентны. Поэтому класс эквивалентности определяется лишь суммарной кратностью прохождения каждого ребра с учетом ориентаций.

Легко переформулировать этот критерий как некоторое решение проблемы тождества слов в группе $S o l_{d}^{2}$ в терминах самих этих слов, т.е. как нормальную форму элементов группы, но приведенный критерий более полезен для дальнейшего.

Рассмотрим теперь пространство бесконечных путей на решетке $\mathbb{Z}^{d}$, начинающихся в нуле. Его можно отождествить с пространством бесконечных последовательностей $S^{\mathbb{N}}$ в алфавите $S$ из образуюших и их обратных. Снабдим его, как и выше, естественной топологией и (бернуллиевской) мерой $\mu^{\infty}$.

Рассмотрим также пространство $F\left(E^{d} ; \mathbb{Z} \cup+\infty\right)$ всех функций с целыми или бесконечными значениями на множестве ребер решетки $E^{d}$ и введем отображение $\Phi$ пространства бесконечных путей $S^{\mathbb{N}}$ в $F\left(E^{d} ; \mathbb{Z}\right)$, сопоставляющее бесконечному пути $\gamma$ и ребру $\rho$ число $\lim _{n \rightarrow \infty} \gamma_{n}(\rho)$, если предел сушествует (т.е. последовательность $\gamma_{n}(\rho)$ стабилизируется), и $+\infty$ - в противоположном случае, здесь $\gamma_{n}$ есть начальньй отрезок пути $\gamma$.

Напомним фундаментальный факт из теории простых случайных блужданий на решетках: если $d=1,2$, то случайное блуждание возвратно, т.е. с вероятностью единица бесконечное число раз проходит через любую вершину и по любому ребру, а если $d>2$, то случайное блуждание невозвратно и, в частности, каждое ребро с вероятностью единица проходится траекторией случайного блуждания конечное число раз (см. [54]). Мы будем далее рассматривать именно этот случай $d>2$. Назовем стабильными функциями функции из $F\left(E^{d} ; \mathbb{Z}\right)$. (Мы исключаем бесконечные значения, так как в силу невозвратности блуждания все значения стабильных функций конечны.) Два бесконечных пути, имеющих при отображении $\Phi$ один и тот же образ (конечный или нет), назовем стабильно эквивалентными. Таким образом, стабильная эквивалентность совпадает с тем определением стабильности, которое рассматривалось в предыдушем параграфе. Наша задача - доказать, что эта эквивалентность совпадает с эквивалентностью, определяемой граничным разбиением $\sigma$ на $S^{\mathbb{N}}$ из $\S 3$. То есть что не существует такой измеримой функции (достаточно рассматривать функции из $L^{2}$ ), которая была бы ортогональна всем стабильным функциям и была бы $\sigma$-измерима, т.е. постоянна на путях, конечные отрезки которых эквивалентны в определенном выше смысле.

ТеОРема 10. 1. При $d \leqslant 2$ пространство классов бесконечных стабильно әквивалентньх путей и граница $\left(\Gamma\left(\right.\right.$ Sol $\left.\left._{d}, \mu\right), \mu_{\sigma}\right)$, где $\mu$-равномерная мера на образующих группь Sol $_{d}$, тривиальнь $(\bmod 0)$, m.е. состоят из одной точки. Точнее, отображсение $\Phi$ переводит почти все пути в тохдественно бесконечную функиию на ребрах.

2. При $d>2$ пространство классов стабильно эквивалентных путей нетривиально и канонически изоморфно границе $\left(\Gamma\left(S_{o}, \mu\right), \widehat{\mu}\right)$. Точнее, отображсние

$$
\Phi:\left(S^{\mathbb{N}}, \mu^{\infty}\right) \rightarrow F\left(E^{d} ; \mathbb{Z}\right)
$$

корректно определено, и для почти всех путей образ есть всюду конечная функиия на ребрах, а проекиия $\Phi$ есть канонический изоморфизм этого пространства и граничь:

$$
\left(\Gamma\left(\operatorname{Sol}_{d}, \mu\right), \widehat{\mu}\right)=\left(F\left(E^{d} ; \mathbb{Z}\right), \Phi\left(\mu^{\infty}\right)\right)
$$


Таким образом, граница Пуассона-Фюрстенберга пары $\Gamma\left(S o l_{d}^{2}, \mu\right)$ как пространство с мерой канонически изоморфно пространству $\left(F\left(E^{d} ; \mathbb{Z}\right), \Phi\left(\mu^{\infty}\right)\right)$ целочисленных функций на ребрах, снабженных мерой-образом.

ДокАЗАТЕльСтво. Мы используем тот же прием, что и в теореме $\S 3$ о гранищах для групп со стабильной нормальной формой, однако пользоваться будем не стабильными нормальными формами, а стабильной эквивалентностью. И здесь достаточно доказать, что пределы функций, зависящих от стабильных координат, исчерпывают все пространство $\sigma$-измеримых (граничных) функций. Предполагая, что такая функция существует и независима от всех стабильных функций, аппроксимируем ее цилиндрическими, т.е. $n$-стабильными функциями, зависящими от стабильных координат путей длины, меньшей $n$ (см. теорему $\S 3$ ). По тем же соображениям, что и в $\S 3$, такие функции должны зависеть только от координат $(\beta(\gamma))$, которые могут при продолжении пути $\gamma$ меняться, и, следовательно, в силу невозвратности расстояние соответствующих ребер $\beta$ от начала координат должно быть больше некоторой константы, зависяшей от $n$ для путей $\gamma$ из множества меры, достаточно близкой к единице, также определяемой выбором $n$. Выбирая, как и ранее, последовательность $n_{k}$ так, чтобы эти множества номеров координат, от которых зависят аппроксиманты, не пересекались, мы получаем противоречие (если функции отличны от константы) с асимптотической независимостью значений функционалов $\beta(\gamma)$ при достаточно далеких друг от друга ребрах $\beta$. Таким образом, функций, отличных от констант, ортогональных всем стабильным функционалам, не существует. В соответствии с этим граница $\Gamma\left(S o l_{d}^{2}, \mu\right)$ отображается в $\left(F\left(E^{d} ; \mathbb{Z}\right), \Phi\left(\mu^{\infty}\right)\right)$, поскольку образ пути при этом отображении зависит лишш от стабильных функционалов от пути, и это отображение есть изоморфизм.

ЗАмечаниЕ 5. Свойства меры $\Phi\left(\mu^{\infty}\right)$, т.е. канонической меры на границе свободной разрешимой групшы, представляют большой интерес. Это мера на пространстве целочисленных функций на ребрах решетки. Ее одномерные распределения (значения на одном ребре) выражаются через разности функции Грина простого $d$-мерного $(d \geqslant 3)$ случайного блуждания в конце и начале ребра. Однако о корреляциях этой естественной меры автору ничего не известно. Эта мера представляется более естественной, чем аналогичная мера на конфигурациях, т.е. на пространстве целочисленных функций на узлах решетки, возникающая в модели сплетения (см. ниже).

Применим этот метод к другим группам. Рассмотрим сплетение $\mathbb{Z}^{d} \imath H \equiv G_{d}(H)$, где $H$ - циклическая группа конечного или бесконечного порядка (это сплетение назьвают теперь лампочной группой - "lamplighter group", поскольку блуждание на ней есть блуждание по решетке с одновременным случайным переключением лампочек в каждом из узлов решетки). Это также разрешимая группа ступени 2 c $d+1$ образуюшей, ее удобно представлять как скрещенное произведение $\mathbb{Z}^{d}$ на пространство $F_{0}\left(\mathbb{Z}^{d} ; H\right)$ всех финитных конфигураций - функций на решетке со значениями в групп $H$. Следовательно, эта группа естественным образом представляется как фактор-группа группы $S o l_{d+1}$. Если $d \geqslant 3$, то граница $\Gamma\left(G_{d}(H), \mu\right)$, әде $\mu-$ равномерная мера на образующих, нетривиальна; это впервые было отмечено в [8]. Легко видеть, что гранища отображается на пространство $F\left(\mathbb{Z}^{d} ; H\right)$ всех конфигураций: это отображение есть просто “финальная” конфигурация, каждому узлу сопоставляется тот элемент группы $H$, который был в момент последнего посещения блужданием 
этого узла. Однако до сих пор оставалось неясным, является ли это отображение изоморфизмом, т.е. всякая ли функция на границе зависит только от финальной конфигурации. Этот вопрос был сведен к некоторой задаче об обычных простых блужданиях на решетке, но решения получено не было. В том случае, когда $H$ - полугруппа, ответ положителен (см. [79]), но метод не применим к случаю группы.

СлЕДСТВИЕ 2. Граница $Г\left(G_{d}(H), \mu\right)$ изоморфна пространству $F\left(\mathbb{Z}^{d} ; H\right)$.

ДокАЗАТЕльство. Можно использовать доказанную вьше теорему для свободной разрешимой группы $S o l_{d+1}$ и тот факт, что сплетение есть ее фактор-группа. Но более поучительно провести аналогичное рассуждение для самого сплетения, используя описанньй метод. Рассмотрим для простоты случай $d=3$ и $H=\mathbb{Z}$ и обозначим сплетение $\mathbb{Z}^{3} \imath \mathbb{Z}$ через $G_{3}$. В этом случае сплетение имеет четыре образующих, первые три из которых коммутируют, и коммутаторы любого элемента из подгруппы $\mathbb{Z}^{3}$, образованной ими, с четвертой образуюшей также коммутируют. Будем отождествлять слова в образуюших с путями на решетке $E^{4}$. Из предыдушего вытекает, что два пути $G_{3}$-эквивалентны тогда и только тогда, когда совпадают проекции концов путей на подрешетку, образованную первыми тремя осями, и алгебраическая сумма кратностей прохождения каждого из ребер, параллельных четвертой оси, одна и та же для обоих путей. Дальнейшее рассуждение в точности повторяет доказательство теоремы для свободной разрешимой группы.

Имевшиеся попытки доказать эту теорему наталкивались на следуюшую сложность: сведение задачи о границе группы $G_{d}$ к блужданию на групе $\mathbb{Z}^{d}$ (а не $\mathbb{Z}^{d+1}$ ) затемняет особую роль четвертой образуюшей, что приводит к необходимости исследовать условньй процесс и доказьвать тривиальность его хвостовой сигма-алгебры, а это требует тех или иных оценок сложных функционалов от траекторий. В проведенном рассуждении различие между образующими прослеживается, как мы видели, очень наглядно. В частности, проекиия кратностей прохождения ребер, параллельных четвертой оси, на решетку, образованную первыми тремя образующими, - и есть финальная конфигурация, о которой шла речь вьше.

Мы не будем останавливаться на других примерах, отметим лишь, что приведенный метод сводит все задачи о границах на конечнопорожденных группах к задачам о тех или иных сигма-алгебрах (а именно, о сигма-алгебрах $G$-эквивалентных путей) для классических простых блужданий на решетках того же ранга, что и число образующих группы. Но не всегда удается легко описать $G$-эквивалентность путей (например, для групп кос). Этот метод также показьвает, что поиск нормальной формы есть выделение какого-то одного пути из класса эквивалентных, а задача о минимальной нормальной форме есть задача изопериметрического типа: найти контур минимальной длинь в данном классе (замкнутых) путей. Для непрерьвной группы Гейзенберга - это классическая изопериметрическая задача. В общем случае они названы изоголономньми (см. [80]) - это задачи о минимуме длины кривой при фиксированных значениях некоторого семейства 1-форм.

Для свободной разрешимой групшы нормальная форма элементов также может быть описана, но гораздо продуктивнее рассматривать в качестве координат не исходные образуюшие, а те, которые рассмотрены выше, - описание границы оказалось не связанным с бесконечными словами и даже не интерпретируется в 
“цилиндрических" (по отношению к $S^{\mathbb{N}}$ ) терминах. Более подробно на этих вопросах мы остановимся в другом месте.

В заключение заметим, что точный подсчет всех трех констант $-v, c, h$ - для свободных разрешимых групп ступени два, видимо, весьма не прост, равно как и явное вычисление значений гармонических функций, - пока неизвестно, преврашается ли в равенство фундаментальное неравенство. Не исключено, что эти константы для свободных разрешимых групп более высоких ступеней аномально близки к константам свободной группы с тем же числом образуюших.

Использованные приемы отыскания границ с помошью пространств путей и сама реализация групп имеют широкую область применений: при отыскании других границ (Мартина), перечислении мер с заданным коциклом, центральных мер и др. могут быть использованы те же средства.

\section{§5. Локальные и локально свободные группы}

\section{1. Определения.}

ОПРЕДЕЛЕНИЕ 13. Локальной группой (алгеброй) L (см. [32], [33]) называется группа, в которой образующие $a_{i}$ линейно упорядочены по типу $\mathbb{Z}, \mathbb{N}$ или по типу отрезка натурального ряда, при этом

$$
a_{i} a_{j}=a_{j} a_{i}, \quad|i-j|>k,
$$

а между образующими $a_{i}, \ldots, a_{i+k}$ имеются некоторые соотношения. Если эти соотношения не зависят от $i$, то группа (алгебра) называется локальной стационарной группой (алгеброй).

Как правило, интересен случай $k=1$, в этом случае коммутируют все несоседние образуюшие. Мы будем рассматривать только этот случай. Если никаких соотношений между соседними образующими нет, то локальная группа (алгебра) называется локально свободной. Мы будем рассматривать локально свободную групу $L F_{n}$ с $n$ образуюшими и точно так же определяемую локально свободную полугруппу $L F{ }_{n}^{+}$с $n$ образующими. Эти группы (полугруппы) являются свободными объектами в многообразии локальных групп (полугрупп).

По указанной причине локально свободная группа $L F_{n}$ накрывает групп кос $B_{n+1}$ c $n+1$ нитями ( $n$ образующими). Напомним, что группа кос есть в наших терминах локальная стационарная группа с соотношением между соседними образующими:

$$
\sigma_{i} \sigma_{i+1} \sigma_{i}=\sigma_{i+1} \sigma_{i} \sigma_{i+1}
$$

Квадраты образуюших $\sigma^{2}$ порождают в группе кос $B_{n}$ подгруппу, изоморфную локально свободной группе. Иначе говоря, между квадратами соседних образующих нет никаких соотношений. Доказательство этого полезного для дальнейшего факта не столь просто, как может показаться из следуюшего примера групшы $B_{3}$, фактор которой по центру есть группа $P S L(2, \mathbb{Z})$, а две образующие переходят в матрицы

$$
\left(\begin{array}{ll}
1 & 1 \\
0 & 1
\end{array}\right), \quad\left(\begin{array}{ll}
1 & 0 \\
1 & 1
\end{array}\right),
$$


квадраты которых образуют, как известно, свободную подгруппу. Случай произвольного $n$ есть специальный вариант гипотезы Титса, который для группы кос доказан в [81], [82].

Нормальньй делитель, порожденньй подгруппой квадратов стандартных образующих в группе кос (т.е. объединение всех подгрупп, сопряженных с этой подгруппой), совпадает с подгруппой крашеных кос. Наш интерес к локально свободной группе связан, в частности, с надеждой на то, что она поможет в изучении границ и асимптотик групп кос и, в частности, стабильных групп кос.

Основные примеры локальных групп и алгебр - группы Кокстера, алгебры Гекке и др. (см. [32], [33]). Легко перенести определения на случай, когда вместо линейного порядка рассматривается произвольньй граф,, вершины которого нумеруют образующие, а пары образующих, не соединенные ребрами, коммутируют. Особенно полезен случай цикла в качестве графа - в этом случае мы называем соответствуюшую локально свободную группу $n$-циклической ( $n$ - число вершин цикла) и обозначаем $L F C_{n}$. Идея локальных групп очень полезна в теории представлений, например, симметрической группы (см. [83]); очевидна ее связь с концепциями теории интегрируемых решеточных моделей и уравнением Янга-Бакстера.

Мы будем рассматривать здесь лиш локально свободные группы $L F_{n}$ и полугруппы $L F_{n}^{+}$со стандартными образующими $g_{1}, \ldots, g_{n}$ и равномерной мерой $\mu$ на них и, в случае группы, их обратных. Легко написать нормальную минимальную форму для элементов локально свободной групшы с образующими. Для этого надо каждое слово записьвать так, чтобы после ненулевой степени каждой из образуюших $g_{i}$ в слове стояла образуюшая с бо́льшим или на единицу меньшим номером. Этим определена нормальная (минимальная) форма в группе (полугруппе). Точно так же определяются бесконечные слова в нормальной форме.

ЛЕмма 11. Эта нормальная форма - стабильна.

Нормальная форма имеет замечательную геометрическую модель - элементы локально свободной групшы или полугрупшы можно представлять себе как груды (этот термин использовался в общей алгебре для объектов, которые редко употребляются, поэтому мы решили именно им перевести принятое в западной литературе слово "heap"). Это понятие возникло в связи со свободной полугруппой [36], но использовалось для других задач (см. [37]).

ОПРЕДЕЛЕНИЕ 14. Грудой назьвается всякое конечное подмножество $H=\{(i, j)\}$ дискретного бесконечного прямоугольника $\{1, \ldots, n\} \times\{1, \ldots, \infty\}, n \in \mathbb{N}$, удовлетворяющее следующим условиям.

Если точка (будем называть ее кирпичом) $(i, j) \in H$, то $(i \pm 1, j) \notin H$ и для $j \geqslant 2$ хотя бы один из элементов $(i-1, j-1),(i, j-1),(i+1, j-1)$ принадлежит $H$. Число $n$ - ширина груды - фиксировано, это число образуюших в группе (полугруппе). При фиксированном $i$ совокупность позиций $\{(i, \cdot)\}$ назовем $i$-й колонкой.

Каждая груда определяет однозначно элемент полугруппы $L F_{n}^{+}$; число кирпичей в груде есть минимальная длина элемента полугрупшы. Поэтому число элементов данной длины есть число груд с данным число кирпичей. Для группы надо определить груды, кирпичи которых могут быть окрашены в один из двух цветов $(+,-)$, и дополнительно потребовать, чтобы цвета кирпичей $(i, j),(i, j+1)$ были одинаковы. 
Комбинаторика груд напоминает комбинаторику диаграмм Юнга. В частности, определяется понятие крыии груды: множества тех ее кирпичей, которые можно удалить, причем остающееся множество по-прежнему есть груда. Это понятие аналогично понятию угла в диаграмме Юнга. Комбинаторика груд является основным техническим средством для получения результатов, приводимых ниже. В терминах нормальной формы крыша состоит из букв слова, которые могут быть сокрашены добавлением справа обратного элемента. Мы отсылаем интересуюшихся к работам [19] и [37], [38].

Модель случайного блуждания на локально свободной группе и полугруппе сводится к модели случайного роста груды, рассмотренной впервые в [19]: независимым (с одинаковыми вероятностями) образом бросаются кирпичи в колонки с соблюдением указанных правил (что-то вроде игры в тетрис). Ищутся вероятностные характеристики случайной груды, и, в частности, оценки размера крьшши дают возможность оценить энтропию и другие нужные константы. Оценка математического ожидания высоты груды является непростой и не до конца решенной задачей.

Как следствие теоремы о гранище групшы с мерой со стабильной нормальной формой (§3) или непосредственно как теорема о границе свободной метаабелевой группы (§4) доказьвается следуюшая теорема.

Tеорема 11. Рассмотрим локально свободную группу (полугруппу) $L F_{n}$ $\left(L F_{n}^{+}\right)$с равномерной мерой $\mu$ на образующих. Граница группь (полугруппь) есть множество бесконечных слов в нормальной форме или, в геометрической формулировке, - множество бесконечных крашеных груд (әруд в случае полугруппв).

Эта модель груд сходна с некоторыми физическими моделями (ASEP) и моделями направленного роста (ср. [19], [84]-[86]), активно изучаемыми в настоящее время.

5.2. Фундаментальное неравенство для локально свободных групп. Мы приведем результаты работы [19], где вычислены, точнее, даны оценки всех трех констант $(v, l, h)$ для локально свободной группы и равномерной меры на естественных образуюших.

ТЕоРема 12. Для локально свободной группы с двумя и более образующими фундаментальное неравенство является строгим (т.е. не является равенством).

Особенность постановки вопросов для локально свободных групп и, более общо, локальных групп [33], в частности, группы кос, в специальном интересе к асимптотике по числу образующих. Оказьвается, такой конечный предел существует для каждой из трех констант. Мы приведем соответствующие оценки. Заметим сначала, что каждая из групп имеет свою "положительную" полугруппу $L F_{n}^{+}$, порожденную положительными степенями образуюших, которая служит базой для предварительных оценок.

ТЕОРемА 13. Значения пределов констант при стремлении числа образующих к бесконечности $(n \rightarrow \infty)$ такови:

1. Логарифмический обвем равен для $L F_{n}^{+}$

$$
v=\ln 4+O\left(n^{-1}\right),
$$


$\partial \Omega я F_{n}-$

$$
v=\ln 7+O\left(n^{-1}\right) .
$$

2. Снос для $L F_{n}$ равен

$$
l=\frac{2-\alpha}{3-\alpha},
$$

әде константа $|\alpha|<1 / 2$, m.е.

$$
3 / 5<l<5 / 7 .
$$

(Снос для полугруппь равен $l=1$, так как в $L F_{n}^{+}$нет сокращений.)

3. Энтропия равна для $L F_{n}^{+}$

$$
h=\ln 3+O\left(n^{-1}\right),
$$

a $\partial \Omega_{s} L F_{n}-$

$$
h=\ln (3-\alpha)+O\left(n^{-1}\right) .
$$

Таким образом, фундаментальные неравенства в обоих случаях строгие при достаточно больших $n$ : для $L F_{n}^{+}$

$$
\ln 3<\ln 4,
$$

а для $L F_{n}-$

$$
\ln (3-\alpha)<\frac{2-\alpha}{3-\alpha} \cdot \ln 7
$$

для $|\alpha|<1 / 2$. Таким образом, константа $q$ из п. 1 равна

$$
q=\frac{(3-\alpha) \ln (3-\alpha)}{(2-\alpha) \ln 7}<1 .
$$

Эти подсчеты существенно используют теоремы, цитированные в предыдущем пункте, в частности, аналог теоремы Шеннона-Макмиллана-Бреймана и др., а также геометрическую модель груд, о которой говорилось в первом пункте.

Из соотношений между группами кос и локально свободными группами, указанных вьшше, можно получить оценки энтропии, сноса и для группы кос, но вопрос о неравенстве для этих групп не решен, и описание границы для локально свободной групшы не помогает явному нахождению границы для группы кос - в отличие от нее в групте кос ни для одной из известных нормальный форм стабилизация не доказана.

Но даже для достаточно хорошо изученной локально свободной группы мы до сих пор не знаем, есть ли в ней образующие, для которых эта константа лучше, чем для естественных образуюших, и тем более неизвестно, является ли эта группа свободноподобной, т.е. есть ли в ней система образуюших, для которой $q_{S}=q=1$.

О чем говорит строгое неравенство для локально свободной групшы? Здесь мы дадим объяснение этому эффекту, которое, по-видимому, имеет общий характер. Это явление, которое можно назвать "запиранием" слов. В чем различие между типичными словами, которые мы получаем в процессе случайного блуждания (метода Монте-Карло), и типичными в смысле равномерного распределения на словах данной длины? Модель груд дает немедленный ответ: в процессе роста случайная груда может 
закрыть (и закрывает) доступ ко многим клеткам, которые могли бы быть заполнены ранее. Иначе говоря, эволюция случайной груды пропускает (с положительной вероятностью) большое число промежуточных возможностей, точнее, "запирает" некоторые клетки, которые уже нельзя заполнить в процессе последующего роста. Поэтому типичная случайная груда имеет больше пустых мест, чем типичная по отношению к равномерной мере, и именно поэтому число типичных случайных слов данной длины экспоненциально мало по сравнению с числом всех слов данной длины. Эксперименты подтверждают различие в плотностях заполнения в том и другом случае.

В этом и состоит физическое объяснение причины неравенства в фундаментальном неравенстве.

Возникает вопрос: как можно исправить случайный механизм, чтобы получить все же равенство в фундаментальном неравенстве? Ответ состоит в том, что случайный механизм должен допускать появление "запертых" слов. При переводе на групповой язык этот шаг означает, что нужно допустить не только приписьвание образующих справа к имеющемуся слову, как в обычном случайном блуждании (правом), но и случайную ВСТАВКУ образующих внутрь слова с соблюдением некоторых правил. Такие блуждания на группах можно назвать блужданиями со вставками.

Разумеется, это резко расширяет возможности случайных механизмов и соответственно усложняет всю теорию таких обобщенных блужданий на группах, полугруппах и т. д. Но в то же время это сближает задачу генерации элементов группы, изучения характеристик их действительно типичных свойств, получение численњых характеристик с новыми проблемами, возникавшими в других областях (ср. [87]). Именно такие блуждания отвечают моделям направленного роста в статистике полимеров (marche dirigée - cм., например, [38]); и, тем самым, модели получают алгебраическое истолкование.

\section{Добавление. \\ О различньх понятиях энтропии; энтропия убывающих последовательностей, вторичная энтропия}

$$
\begin{array}{r}
\text { “............. Энтропия! } \\
\text { Энтропия ............." } \\
\text { Тимур Кибиров. }
\end{array}
$$

В этом добавлении после нескольких кратких замечаний об истории энтропии в теории меры и функциональном анализе мы приводим несколько новых понятий энтропии, определяемых по обшей схеме.

А.Н. Колмогоров первым по-настояшему осознал фундаментальную роль шенноновской теории информации для всей математики и дал ей обшематематическое, универсальное употребление. Самым значительным применением энтропии в математике является, несомненно, его понятие энтропии в метрической теории динамических систем. Чуть позже это привело и к понятию топологической энтропии. Он же сделал $\varepsilon$-энтропию компактов, появившуюся еще в $30-$ х гг., но не ставшую до него обшепринятым инструментом исследования, средством изучения бесконечномерных и функциональных пространств, их размерностей и их аппроксимаций. Все последующие поня- 
тия энтропии могут считаться потомками колмогоровской и шенноновской энтропий в разных областях.

Колмогоровская энтропия динамической системы, введенная более сорока лет назад [88], [89], поставила теорию динамических систем и эргодическую теорию на совершенно новьй уровень. В одном из своих публичных докладов В. А. Рохлин сказал: "Каждый может сделать свою науку модной, надо только выдумать что-либо подобное энтропии Колмогорова". Действительно, эргодическая теория и теория динамических систем из сравнительно периферийной области оказались на долгое время и, пожалуй, до сих пор остаются в центре внимания большинства математиков. Конечно, тому имеются и другие веские причины, это, в первую очередь, их востребованность в разнообразных приложениях, а также многие другие открытия (KАМ-теория, гиперболическая теория, теория Орнштейна, связи со статфизикой, вычислительные вопросы и т. д.). В.А. Рохлин к началу пятидесятых годов перестал заниматься эргодической теорией, в которой ему в 40-е гг. принадлежали крупные достижения. Он целиком переключился на гладкую топологию, где совершил замечательные открытия. Но, по его собственному признанию, открытие Колмогорова (кстати, впервые доложенное на семинаре Рохлина в МГУ) произвело на него очень сильное впечатление, и он надолго, почти до конца 60-х гг., сосредоточился на изучении эргодической теории и, в первую очередь, на энтропийной теории. В. А. Рохлину и Я. Г. Синаю безусловно принадлежат главные и наиболее глубокие факты энтропийной теории [68], [90]-[92], в которую, конечно, внесли вклад многие: М. С. Пинскер, Л. М. Абрамов, Д. З. Аров, С.А. Юзвинский и другие. Блестяшие обзоры В. А. Рохлина в "Успехах" [90], [68] до сих пор могут читаться как руководства по этой теме. Во втором из них было дано первое замкнутое изложение того состояния энтропийной теории, которое было достигнуто к тому времени. К сожалению, ни им, ни другими создателями теории не была написана специальная монография по энтропийной теории динамических систем, дело ограничилось лишш обшими сведениями в книгах [93], [94] и несколькими обзорами. Последуюшие обстоятельные книги [95]-[97] и др. написаны людьми, не участвовавшими непосредственно в событиях, что налагает отпечаток на их стиль.

После работ 50-х гг. А.Н. Колмогорова и работ его продолжателей в математический обиход вошло своеобразное әнтропийное мыиление, т.е. определенньй прием построения инвариантов экспоненциального типа в различных ситуациях. Такие инварианты, если они сушествуют, как правило, устойчивы и потому вычислимы. Их интерпретация может быть очень разной - скорость передачи информации, показатель убывания вероятности, скорость перемешивания или скорость роста и т. д. Эти инварианты связаны с показателями Ляпунова, действием, функциями роста, хаусдорфовой размерностью мер, они могут служить средством различения взаимно сингулярных мер и т.п. Энтропийная теория действия аменабельных групп с инвариантной мерой также получила значительное развитие (см. [98]), однако за пределами этого класса групп положение остается неясным.

В этой статье мы имели дело с энтропией случайных блужданий, фактически характеризующей информацию, содержащуюся в одной букве случайного слова, представляюшего данный элемент группы (при выбранной системе образуюших и их вероятностей), или, иначе говоря, экспоненциальную скорость того, как "размазываются" по группе свертки данной меры. Мы придали этой энтропии более широкий смысл, определив ее как колмогоровскую энтропию, но уже для полиморфизмов - многознач- 
ных преобразований, и связали энтропийньй критерий тривиальности гранищы с полной положительностью энтропии преобразований. Стоит отметить, что в некотором отношении колмогоровская энтропия преобразования как раз и измеряет близость его к многозначному (хаотичность преобразования может быть охарактеризована как существенная многозначность фактор-полиморфизмов по конечным разбиениям).

Очень близко в техническом отношении к колмогоровскому определению находится понятие энтропии фильтраций (убывающих последовательностей разбиений), в частности, наиболее изученная энтропия диадических последовательностей см. [39]. Здесь, как и в определяемой далее вторичной әнтропии динамической системьи, энтропия выступает как показатель экспоненциального разброса в асимптотике условных мер. В этом добавлении мы даем новую трактовку энтропии последовательностей измеримых разбиений, годную и для неоднородных разбиений и использующую одновременно и $\varepsilon$-энтропию компактов, и по аналогии с этим определяем вторичную энтропию случайных регулярных процессов. Следует заметить, что все виды энтропий (быть может, пока за исключением энтропий в некоммутативных теориях) определяются по общей схеме: с данньп объектом тем или иньм образом ассоциируются допустимые возрастающие последовательности конечных разбиений и выгисляется предел их нормированных энтропий, а затем берется супремум по всем допустимым последовательностям, который и назьвается энтропией данного объекта. При этом для приложений существенно, чтобы предел зависел непрерьвно от возрастаюшей последовательности, что и дает возможность вычислять энтропию. Первый пример этой схемы и есть энтропия Колмогорова в варианте, предложенном Синаем. В эту же схему вкладьвается буквальньм образом (без взятия супремума) энтропия случайных блужданий в том варианте, который описан в 3 , а также и нижеследующие определения.

\section{§. Энтропия дискретных фильтраций}

Дискретной фильтрацией в теории случайных процессов назьвают убывающую последовательность сигма-алгебр или, на более удобном для нас языке, убъвающую последовательность измеримьх разбиений пространства Лебега. Обозначим такую последовательность $\left\{\xi_{n}\right\}_{n \in \mathbb{N}}$. Здесь $\xi_{n+1} \prec \xi_{n}$, а $\xi_{0}$ - разбиение на отдельные точки mod 0 пространства Лебега $(X, \mu)$. Мы не налагаем никаких требований на условные меры разбиений, наиболее интересные частные случаи, с которых начиналась эта теория (см. библиографию в [39]), - диадический (условные меры разбиения $\xi_{n}$ равномерны и каждый элемент содержит $2^{n}$ точек) и непрерывньй (условные меры всех разбиений $\xi_{n} / \xi_{n-1}$ непрерьвны). Мы не предполагаем также и эргодичности, т.е. того, что измеримое пересечение последовательности $\left\{\xi_{n}\right\}_{n \in \mathbb{N}}-$ тривиально. Важный факт теории фильтраций состоит в том, что существует континуум попарно неизоморфных однородных диадических эргодических последовательностей, и потому встает задача классификации фильтраций. Один из естественных инвариантов энтропия диадической последовательности $h\left(\left\{\xi_{n}\right\}_{n \in \mathbb{N}}\right)$, введенная в [69], [99], см. также [100].

Приводимое ниже новое определение энтропии в силу некоторых комбинаторных соображений совпадает для диадических последовательностей при экспоненциальной нормировке (скейлинге) с прежней энтропией $h\left(\left\{\xi_{n}\right\}_{n \in \mathbb{N}}\right)$ диадических последо- 
вательностей, как она была введена в [99] и подробно рассмотрена в [39]. Но это определение допускает уже различные скейлинги и, кроме того, пригодно и для неоднородных последовательностей. С другой стороны, оно использует понятие $\varepsilon$-энтропии компактного пространства мер и позволяет рассматривать произвольные асимптотики роста, а это, в свою очередь, дает новые возможности даже для диадических последовательностей, для которых старая энтропия обрашалась в нуль. Заметим, что для определения энтропии диадических (однородных) последовательностей может быть использована колмогоровская энтропия действия диадической ( $p$-адической) группы: она, как показано в [100], есть инвариант не только действия диадической групшы, ассоциированного с последовательностью, но и самой диадической последовательности, и при этом она совпадает с нашей энтропией $h\left(\left\{\xi_{n}\right\}_{n \in \mathbb{N}}\right)$. Однако этот способ использования колмогоровской энтропии действия никак не обобщается на неоднородньй случай, так как никакого естественно ассоциированного с общего вида убывающей последовательностью действия определить нельзя. Метод использования итерированных метрик Канторовича-Рубинштейна (KP), предложенньй в [69] и использованный в формулировке критерия стандартности, позволяет рассмотреть самьй общий случай.

Напомним сначала построение КР-метрики. Пусть задан метрический компакт $(K, r)$, где $r$ - метрика (или даже полуметрика) на $K$. По метрике (полуметрике) $r$ на $K$ можно построить метрику (соответственно полуметрику) $\rho_{r}$ на $V(K)-$ пространстве вероятностных борелевских мер. Эта транспортная, или КР-метрика, введенная впервые Л. В. Канторовичем [101] и исследованная в совместной статье с Г.Ш. Рубинштейном [102], переоткрывалась в десятках работ на самые различные темы, она оказалась необычайно полезной во многих областях: в теории меры, эргодической теории, статистике, статфизике и пр. Она определяется как

$$
\rho_{r}\left(\mu_{1}, \mu_{2}\right)=\inf \mathrm{E}_{\psi} r\left(x_{1}, x_{2}\right)
$$

где инфимум берется по всем вероятностным мерам $\psi$ на произведении $K \times K$ с заданными маргинальньми мерами (проекциями на сомножители) $\mu_{1}$ и $\mu_{2}$, а $\mathrm{E}_{\psi}$ - знак математического ожидания по мере $\psi$. Иначе говоря, это инфимум по всевозможным совместным распределениям математических ожиданий расстояния между двумя случайными величинами, принимающими значения в данном метрическом пространстве и имеющими заданные распределения.

Эта конструкция перехода от $(K, r)$ к $\left(V(K), \rho_{r}\right)$ допускает итерирование: в работах автора [39], [69] введена так называемая башшя мер $V^{k}=V\left(V^{k-1}\right), k=1,2, \ldots$ ("меры на мерах"), с соответствующими итерациями КР-метрик и ее проективньй и индуктивные пределы. Здесь мы не будем обрашаться к этим понятиям во всей их общности и определим итерации непосредственно, используя последовательность разбиений. В работе [39] содержится более полная информация об этой конструкции.

Рассмотрим метрический компакт с мерой и метрикой $(X, \mu, r)$. Предположим, что имеется некоторое измеримое разбиение $\xi$ пространства $(X, \mu, r)$, тогда мы можем ввести новую допустимую полуметрику $\rho_{\xi, r}$ на том же пространстве $(X, \mu)$ следуюшим образом:

$$
\rho_{\xi, r}(x, y)=\rho_{r}\left(\mu^{x}, \mu^{y}\right)
$$


где $\mu^{z}$ есть условная мера на элементе этого разбиения $\xi$, содержашем точку $z$, а $\rho_{r}-$ $\mathrm{KP}$-метрика на мерах, заданных на $(X, r)$. Корректность этого определения вытекает из того, что условные меры определены для почти всех элементов разбиений, а элементы разбиений как подмножества в $(X, r)$, снабжены (полу)метрикой. Заметим, что $\rho_{\xi, r}$, фактически, есть метрика (полуметрика) на фактор-пространстве $X / \xi$, заданная почти всюду. Таким образом, метрика на условных мерах определяет полуметрику на самом пространстве. Если на $(X, \mu, r)$ задана убывающая последовательность разбиений, то на $(X, \mu)$ возникает последовательность полуметрик

$$
\rho_{1}=\rho_{\xi_{1}, r}, \quad \rho_{n}=\rho_{\xi_{n}, \rho_{n-1}}, \quad n=2,3, \ldots
$$

Эта последовательность полуметрик - $\left\{\rho_{n}\right\}$ - определяется по исходной метрике $r$ и последовательности разбиений $\left\{\xi_{n}\right\}$.

Теперь напомним, что такое $\varepsilon$-энтропия меры $\nu$ в метрическом (полуметрическом) пространстве $(Y, r)$, по существу, введенная Шенноном:

$$
H_{\varepsilon, r}(\nu) \equiv \inf \left\{H(\alpha): \rho_{r}(\nu, \alpha)<\varepsilon\right\},
$$

где инфимум берется по всем дискретным мерам $\alpha$ на $Y$ из $\varepsilon$-окрестности (в смысле КР-метрики) меры $\nu$, а $H$ - обычная энтропия дискретной меры. Применяя это понятие к нашему случаю, мы получим функцию от $(n, \varepsilon)$, зависяшую от последовательности разбиений, которую мы фиксируем, и от исходной метрики $r$ :

$$
\varphi(\varepsilon, n, r)=H_{\varepsilon, \rho_{n}}(\mu)
$$

Нам придется в дальнейшем варьировать метрику на фиксированном пространстве с мерой в противоположность традиции, в силу которой, наоборот, фиксируют метрический компакт и на нем рассматривают различные борелевские меры. Поэтому дадим определение допустимой метрики на пространстве Лебега.

ОПРЕДЕЛЕНИЕ 15. Допустимой полуметрикой (в частном случае- метрикой) на пространстве Лебега $(X, \mu)$ назьвается полуметрика (соответственно метрика), определенная на подмножестве полной меры и обладающая следующими свойствами:

а) она измерима как функция двух переменных на $(X \times X, \mu \times \mu)$, в частности, шары с центром в почти каждой точке суть измеримые множества, а разбиение $\omega$ на классы точек с попарно нулевьм расстоянием (в случае полуметрики) является измеримым разбиением;

б) подмножество полной меры в пространстве $X$, снабженное этой метрикой (в случае полуметрики - фактор-пространство $X / \omega$ с фактор-метрикой), есть компакт.

Прием варьирования допустимых метрик на фиксированном пространстве Лебега, по-видимому, недооценивался до сих пор. Определим теперь с его помощью метрический инвариант последовательности разбиений-функцию $\left\{\xi_{n}\right\}(\varepsilon)$, которая будет строиться по некоторой растушей последовательности положительных чисел $\left\{c_{n}\right\}$ - скейлингу, при этом ее выбор однозначно диктуется самой последовательностью разбиений: 
ОПРеДЕЛЕНИЕ 16. Пусть $\left\{\xi_{n}\right\}_{n \in \mathbb{N}}-$ последовательность измеримых разбиений и $r$ - допустимая полуметрика на пространстве $(X, \mu)$. Выберем скейлинговую последовательность положительных чисел $\left\{c_{n}\right\}$ так, чтобы для достаточно малых $\varepsilon \geqslant 0$ существовал предел

$$
h^{(r)}\left(\varepsilon,\left\{\xi_{n}\right\},\left\{c_{n}\right\}\right) \equiv \lim _{n \rightarrow \infty} \frac{\varphi(\varepsilon, n, r)}{c_{n}}
$$

(например, можно взять $c_{n} \equiv \varphi(\widehat{\varepsilon}, n, r)$ для некоторого фиксированного малого $\widehat{\varepsilon}$ и какой-либо полуметрики $r)$.

$\varepsilon$-энтропией последовательности измеримых разбиений $\left\{\xi_{n}\right\}_{n \in \mathbb{N}}$ со скейлингом $c_{n}$ назьвается функция

$$
h\left(\varepsilon,\left\{\xi_{n}\right\},\left\{c_{n}\right\}\right)=\sup h^{(r)}\left(\varepsilon,\left\{\xi_{n}\right\},\left\{c_{n}\right\}\right)
$$

где супремум берется по всем допустимым полуметрикам $r$ на пространстве $X$.

ЗАмЕчАниЕ 6. 1. Существование скейлинга, годящегося для всех полуметрик, требует доказательства, но в случае его существования выбор $\left\{c_{n}\right\}$, при котором предел конечен, единствен с точностью до эквивалентной последовательности. Поэтому указание на $\left\{c_{n}\right\}$ можно опустить.

2. Достаточно варьировать $r$ лишь по вырожденным полуметрикам вида: $r(x, y)=$ $\inf _{i=1, \ldots, n}\left|f_{i}(x)-f_{i}(y)\right|$, где $f_{i}$ - конечньй набор измеримых функций. Более того, можно доказать, что супремум реализуется, например, уже на евклидовой метрике (если отождествить $(X, \mu)$ с единичным отрезком), но вычисления удобнее проводить на вырожденных полуметриках.

3. Как обычно, важен лишь росток функции $h$ в нуле по $\varepsilon$. В некоторых случаях функция не зависит от $\varepsilon$, если $\varepsilon$ достаточно мало. В этом случае инвариант есть число. Именно это имеет место в случае диадических последовательностей и скейлинга $2^{n}$, как проверил студент А. Д. Горбульский, можно даже менять $\varepsilon$ вместе с $n$, а именно, положить $\varepsilon_{n}=2^{-n}$, и тогда определение вьше совпадает со старьм определением энтропии в [39].

ТЕОРема 14. Пусть $\left\{\xi_{n}\right\}$ - диадическая последовательность измеримых разбиений, $c_{n}=2^{n}$. Тогда

$$
\lim _{\varepsilon \rightarrow 0} \lim _{n \rightarrow \infty} \frac{\varphi(\varepsilon, n, r)}{2^{n}}=h^{(r)}\left(\left\{\xi_{n}\right\},\left\{2^{n}\right\}\right)=\lim _{n \rightarrow \infty} \frac{\varphi\left(2^{-n}, n, r\right)}{2^{n}}
$$

и, следовательно, переход $к$ супремуму по $r$ в левой и правой частях равенства приводит к соотношению:

$$
h\left(\varepsilon,\left\{\xi_{n}\right\},\left\{2^{n}\right\}\right)=\bar{h}\left(\left\{\xi_{n}\right\}\right),
$$

где правая часть - $\bar{h}\left(\left\{\xi_{n}\right\}\right)$ - есть әнтропия диадической последовательности в смисле [39], а левая не зависит от в в достаточно малой окрестности нуля.

С очевидньми изменениями то же верно для любых однородных $\left(\left(r_{n}\right)\right.$-адических $)$ последовательностей. С другой стороны, для диадических последовательностей, у которых энтропия равна нулю, возможен иной скейлинг, дающий ненулевую энтропию в 
смысле данного выше обшего определения. Некоторая зависимость от $\varepsilon$ может в этом случае стать необходимой.

Недавний пример [103] нестандартной последовательности разбиений, получающейся из стандартной диадической последовательности изменением плотности меры, как оказалось, имеет положительную энтропию в смысле данного определения. Это фактически прямо следует из вычислений, сделанных в [103], и означает, что энтропия не есть инвариант типа меры (т.е. не сохраняется при преобразованиях с квазиинвариантной мерой). Любопытно, что обращение этого примера - невозможно: превратить диадическую последовательность с положительной энтропией в последовательность с нулевой энтропией с помощью изменения плотности меры - нельзя.

Относительно неплохо изучен еще один класс примеров, в котором энтропия со скейлингом $c_{n}=\left\{2^{-n}\right\}$ равна нулю, но энтропия со скейлингом $c_{n}=\sqrt{n}$ разделяет диадические последовательности. Мы имеем в виду прошлое процесса $\left(T, T^{-1}\right)$, где $T$ - автоморфизм Бернулли. Как доказал С. Каликов, этот автоморфизм не является свободно бернуллиевским (loosely Bernoulli) и тем более бернуллиевским. Поэтому возник вопрос об инвариантах прошлого этого процесса. Гипотеза о том, что эта диадическая последовательность нестандартна, была довольно давно высказана автором и сушественные (энтропийные) доводы в ее пользу были приведены в неопубликованной рукописи (Беркли, 1995). Именно в связи с этим примером автор предложил общее определение энтропии, которое приведено вьше. Полное доказательство нестандартности этой последовательности, основанное на критерии стандартности из [39], дано в [104]. Энтропия как инвариант последовательности в [104] не используется.

\section{$\S$ 7. Вторичная энтропия}

Вторичная энтропия измеряет "случайность случайности” $K$-автоморфизмов и соответствующих стационарных процессов. Она строится точно так же, как и энтропия убьвающих последовательностей, определенная вьше, но по более простой метрике. Если зафиксировать течение процесса до некоторого момента (т.е. зафиксировать прошлое), то распределение вероятности на состояниях будущего, вообще говоря, будет различньм в зависимости от прошлого. Для классической последовательности независимых это распределение одно и то же. Оказывается, для процессов, неудачно названных “очень слабо бернуллиевскими” (=VWB-процессов), случайность будущего, точнее, распределение на будущем, - “детерминировано” в том смысле, что эти распределения мало отличаются в определенной метрике для почти всех возможных течений прошлого случайного процесса. Инвариантность этого свойства образующей - один из главных результатов Орнштейна [105]: по его теореме любая конечная образуюшая бернуллиевского автоморфизма является VWB, поэтому распределения на будущем почти совпадают в смысле КР-метрики. Это свойство аналогично стандартности убываюшей последовательности в силу критерия стандартности (см. ниже). В предлагаемых далее терминах это означает, в частности, что вторичная энтропия равна нулю с любым скейлингом. В то же время, для общих, т.е. небернуллиевских, $K$-автоморфизмов, впервые открытых также Д. Орнштейном в начале 70 -х гг. (см. [40]), случайность будущего может быть существенно различной в зависимости от прошлого. В этих случайных процессах проявляется некоторая небуквальная детерминированность: фиксированное прошлое не определяет однозначно будушего, но 
определяет распределение вероятностей на будушем, которое сушественно зависит от прошлого. Вторичная энтропия как раз и измеряет разброс в той же метрике условных мер на будущем при различных прошлых, точно так же, как энтропия убьвающей последовательности измеряла разброс итерированных метрик на элементах разбиений. Иначе говоря, подобно тому, как классическая энтропия измеряет неопределенность, содержащуюся в передаваемом случайном тексте, вторичная энтропия измеряет неопределенность, содержащуюся в самом распределении вероятностей случайного текста.

Наиболее убедительный пример небернуллиевского $K$-автоморфизма дает случайное блуждание по траекториям стохастической системы, например, исследованное С. Каликовым в его известной работе [106] блуждание с переходньми вероятностями $\operatorname{Prob}\{x \rightarrow T x\}=\operatorname{Prob}\left\{x \rightarrow T^{-1} x\right\}=1 / 2$ по траекториям автоморфизма Бернулли $T$. Такие (на жаргоне "(T, $\left.T^{-1}\right)$-inverse") и чуть более общие примеры блужданий по траекториям динамических систем представляют большой интерес с разных точек зрения и, в частности, в связи с блужданиями в случайных средах. Это - косые произведения и, одновременно, марковские процессы, поэтому они задаются полиморфизмом. Пример Каликова имеет энтропию, равную единице, и является $K$-автоморфизмом, но он не является не только бернуллиевским, но даже "свободно бернуллиевским" (loosely Bernoulli), как уже упоминалось. Это обстоятельство достойно внимания: случайное блуждание по траекториям самого случайного автоморфизма - не является “абсолютно случайным". Отметим, что аналогичное $\left(T, T^{-1}\right)$-преобразование - случайное блуждание по траекториям автоморфизма $T$ с дискретным спектром, определяет, в противоположность предыдушему, автоморфизм Бернулли [107], так же как и блуждание по траекториям бернуллиевского действия свободной группы, но уже по другой причине [39], [69]. В двух последних примерах диадические последовательности прошлых совершенно различны - в первом случае она стандартна, и даже сам эндоморфизм изоморфен бернуллиевскому (Parry, Rudolf-Hoffman), а во втором - нестандартна и имеет бесконечную энтропию; в случае же примера Каликова последовательность прошлых - нестандартна ([104] см. вьше), но ее обьчная энтропия как диадической последовательности - равна нулю.

Перейдем к точным определениям. Пусть $\left(y_{n}, n \in \mathbb{Z}\right)$ - стационарный случайный процесс с конечным множеством состояний $A=(a, b, \ldots, c)$, а $T$ - правьй сдвиг в пространстве $X=\prod_{i \in \mathbb{Z}} A$ траекторий (т.е. $T$ - автоморфизм пространства Лебега $(X, \mu)$, где $\mu$ - мера, определяемая процессом, и выделена конечная двусторонняя образующая $T$, тем самым, $T$ имеет конечную энтропию). Обозначим через $\eta$ эту конечную образуюшую, т.е. разбиение на траектории с одной и той же нулевой координатой, и через $\left(\xi_{n}\right)=\bigvee_{k \leqslant n} T^{k} \eta$ - инвариантное относительно $T$ разбиение пространства $(X, \mu)$ на классы траекторий с совпадаюшими координатами, номера которых не превьшают $n$. В соответствии с обшепринятыми определениями $\xi_{0}$ есть $K$-образующая, а $T-K$-автоморфизм (см. [68]), иначе говоря:

$$
\bigwedge_{n<0} T^{n} \xi_{0}=\nu
$$


где $\nu$ - тривиальное разбиение, и

$$
\bigvee_{n>0} T^{n} \xi_{0}=\varepsilon
$$

где $\varepsilon-$ разбиение $\bmod 0$ на точки.

Рассмотрим на множестве $A$ дискретную метрику $d(x, y)=\delta(x, y)$ и метрику Хэмминга $r_{n}$ на множестве $X_{n}$ конечных слов длины $n, X_{n}=\prod_{1 \leqslant i \leqslant n} A$, т.е.

$$
r_{n}\left(\left(y_{1}, \ldots, y_{n}\right),\left(z_{1}, \ldots, z_{n}\right)\right)=\frac{\left|\left(i: y_{i} \neq z_{i}\right)\right|}{n} .
$$

На пространстве вероятностных мер $V\left(X_{n}\right)$ на $X_{n}$ введем КР-метрику $\rho_{n} \equiv \rho_{r_{n}}$. Обозначим через $\mu_{(0, \infty)}$ проекцию меры $\mu$ на $\prod_{i \leqslant 0} A \equiv X_{-\infty}^{0}$.

Рассмотрим следующее отображение: $F_{n}: X_{-\infty}^{0} \rightarrow V\left(X_{n}\right)$, сопоставляющее почти каждой (по мере $\left.\mu_{(0, \infty)}\right)$ последовательности $\left(y_{i}\right)_{i \leqslant 0}$ (т.е. “прошлому") условную меру на множестве слов $\left(y_{i}\right)_{i=1, \ldots, n}$ ("будушем продолжительности $n$ "). Отображение $F_{n}$ переводит меру $\mu_{(0, \infty)}$ в некоторую меру $M_{n}=F_{n}\left(\mu_{(0, \infty)}\right)$ на $V\left(X_{n}\right)$; вычислим ее $\varepsilon$-энтропию как меры на компакте $V\left(X_{n}\right)$, снабженном КР-метрикой $\rho_{n}$,

$$
H_{\varepsilon, \rho_{n}}\left(M_{n}\right)
$$

(см. вьше определение энтропии убывающей последовательности, как и там, мы фиксируем последовательность $\varepsilon_{n}$ ). Сохраним эти обозначения в нижеследующем определении.

ОПРЕДЕЛЕНИЕ 17. Вторичной энтропией $K$-автоморфизма $T$ относительно конечной двусторонней образующей $\eta$ со скейлингом $c_{n}$ назьвается предел

$$
\operatorname{sh}(T, \eta)(\varepsilon)=\lim _{n \rightarrow \infty} c(n)^{-1} H_{\varepsilon, \rho_{n}}\left(M_{n}\right) .
$$

Вторичной энтропией $K$-автоморфизма $T$ со скейлингом $c_{n}$ назьвается функция

$$
\operatorname{sh}(T)(\varepsilon)=\sup _{\eta} \operatorname{sh}(T, \eta)(\varepsilon)
$$

где супремум берется по всем конечным двусторонним образующим $\eta$ автоморфизма $T$, а скейлинг фиксирован.

Скейлинг $c(n)$ в определении вторичной энтропии $s h(T, \eta)$ можно варьировать так же, как и в определении энтропии последовательности в предыдущем параграфе; мы не указываем его в обозначении $s h(T, \eta)$, поскольку нетривиальный предел получается разве лишь для одного с точностью до асимптотической эквивалентности его выбора.

Вторичная энтропия есть функция от $\varepsilon$, и, как обычно, нас интересует лишш ее росток в нуле, а не вся функция. В некоторых случаях она не зависит от $\varepsilon$ при малых значениях, т.е. является числовым инвариантом. Вторичная энтропия претендует на начальную классификацию небернуллиевских $K$-автоморфизмов, в связи с чем возникает много новых вопросов. Например, каким может быть скейлинг $c(n)$ при $n \rightarrow \infty$ 
в целом для разных автоморфизмов, при котором вторичная энтропия автоморфизма конечна. Ж.-П. Тувено обратил мое внимание на то, что сверхэкспоненциальный рост $c(n)$ невозможен для автоморфизмов, для которых доказана слабая гипотеза Пинскера - т.е. представление автоморфизма в виде прямого произведения автоморфизма Бернулли на автоморфизм с произвольно малой энтропией. Поэтому эта, много лет стоящая, проблема может быть сформулирована как проблема построения (или доказательства его невозможности) примера со сверхэкспоненциальным ростом вторичной энтропии.

В примере Каликова рост последовательности $\{c(n)\}$, по-видимому, есть $\sqrt{n}$, что мы сформулируем в виде обшего предположения ниже.

ГИПОТЕЗА 1. 1. Вторичная энтропия автоморфизма Т с данным скейлингом для данной двусторонней K-образующей $\eta$ положстельна (как функиия от в при мальх значениях) тогда и только тогда, когда положительна (тоже как функиия $\varepsilon)$ әнтропия последовательности прошльх $\left\{T^{-n} \varepsilon\right\}$ с тем же скейлингом. Например, в случае автоморфизма Каликова этот скейлинг - $\{\sqrt{n}\}$.

2. В частности, әнтропия последовательности прошльх (со скейлингом $n^{-1}$ ) равна нулю для бернуллиевских автоморфизмов с произвольной конечной К-образующей.

Сделанное предположение об идентичности скейлингов для вторичной энтропии и для последовательности прошлых базируется на том, что, фактически, для последовательностей прошлого, т.е. для стационарных убывающих последовательностей разбиений, различие между энтропиями, введенными здесь, лишш в том, как определяется метрика в пространстве условных мер: в первом случае - это итерированная КР-метрика, а во втором - это КР-метрика, построенная по метрикам Хэмминга. Итерирование - более сложная процедура, чем увеличение длины слова и соответствуюшее ему изменение метрики Хэмминга. Впрочем, взаимоотношения между этими метриками довольно запутанные.

С другой стороны, в недавней работе К. Мартон и П. Шилдса [41] построен наибольший из известных скейлингов (в других терминах), а именно, оцениваемьй снизу линейным ростом с произвольным коэффициентом. Для убывающих последовательностей скейлинг может быть экспоненциальным - именно так было в моих первых примерах - но, по-видимому, здесь проявляется различие между задачами или, в терминах теории колмогоровских автоморфизмов, - различие между имеющейся теорией Орнштейна для конечной образующей, т.е. процессов с конечным множеством состояний, и пока отсутствуюшей теорией процессов с произвольньм множеством состояний. Дело в том, что положительность вторичной энтропии свидетельствует, что процесс (с конечным числом состояний) не бернуллиевский, т.е. не имеет VWB-образующей, но для произвольного множества состояний положительность вторичной энтропии даже с экспоненциальным скейлингом не противоречит бернуллиевости и говорит лишш о качестве образующей. В упомянутом примере экспоненциальность скейлинга в определении энтропии последовательности прошлых процесса была использована для доказательства нестандартности этой последовательности, т.е. некоторого свойства образующей. Такие образующие либо имеют немаксимальную энтропию, либо отличаются другими нерегулярными свойствами. Как уже упоминалось, эти факты означают, что теория инвариантных разбиений для общего процесса (с бесконечной 
энтропией) должна сильно отличаться от орнштейновской теории, эта теория все еще не разработана.

Подытожим сделанные в добавлении замечания. Главный смысл параллели между вторичной энтропией и энтропией последовательности прошлых не только в технической схожести, но в том, что оба понятия претендуют на то, чтобы быть инвариантами автоморфизмов. Более подробно, последовательность прошлых одностороннего стационарного случайного процесса задает стационарную убьвающую последовательность измеримых разбиений, уже метрический тип последовательности $\left\{T^{-n} \varepsilon\right\}$ сам по себе есть метрический инвариант одностороннего сдвига. Эту идею неоднократно подчеркивал В. А. Рохлин. В частности, энтропия последовательности есть характеристика эндоморфизма. Легко видеть, что обыгная энтропия может принимать на них только два значения - нуль и бесконечность. Однако значения введенной здесь вторичной энтропии со скейлингом уже не стеснено какими-то ограничениями и может быть использовано для нахождения новых инвариантов эндоморфизмов. Но наибольший интерес представляют инварианты двустороннего сдвига. Дело в том, что выбор образующей может сильно изменить последовательность прошлых, и глубокий вопрос в том, какие инварианты прошлого являются инвариантами не только эндоморфизма, но и автоморфизма, т.е. не зависят от того, как выбрана образуюшая. Например, стандартность последовательности прошлых в смысле [39] может иметь место для одной образующей и не иметь для другой - уже первый пример нестандартной последовательности [69] был построен как прошлое автоморфизма Бернулли с бесконечной энтропией относительно неэкстремальной образующей. (Кстати, как замечено было позже, вариация этого примера доказывает гипотезу Синая-Рохлина [68] о том, что энтропия $h\left(\xi \mid T^{-1} \xi\right)$ может принимать любые промежуточные значения между 0 и $h(T)$ на $K$-образуюших.) Недавние примеры Рудольфа-Фельдмана-Хофмана показывают, что у бернуллиевских автоморфизмов с конечной энтропией тоже могут быть образуюшие с нестандартным прошлым. В то же время стандартность (и, следовательно, равенство нулю энтропии) последовательности прошлых может иметь место и для небернуллиевских $K$-автоморфизмов.

Таким образом, тип прошлого как тип убьвающей последовательности разбиений, как видно, не является инвариантом автоморфизма. Но некоторые инварианты последовательностей прошлого, такие как энтропия, являются, по-видимому, инвариантами автоморфизма, именно это есть содержание второго пункта гипотезы выше. Если это так, то мы получаем новый способ классифицировать автоморфизмы и их образуюшие, рассматривая все более и более тонкие свойства последовательности прошлых. Варьируя метрики в пространстве условных мер, можно по тому же образцу строить и иные понятия энтропии. Один такой пример метрики хорошо известен - это $f$-метрика (см. [107], [108]), приводящая к понятию свободной бернуллиевости ("loosely Bernoulli"). Эта метрика также приводит к другому (более широкому) понятию вторичной энтропии. Продолжая аналогию между теорией Орнштейна и теорией убываюших последовательностей прошлого, заметим, что условие VWB Opнштейна в точности параллельно стандартности: критерий стандартности [39] и теорема Орнштейна утверждают, что в обоих случаях идет речь о сходимости последовательности случайных условных мер в соответствующих метриках (итерированной КР или Хэмминга) к вырожденной мере. То же можно сказать и о свободной бернуллиевости, если использовать вместо метрики Хэмминга $f$-метрику. Таким образом, 
вторичная энтропия (как и энтропия последовательностей прошлого) расщепляет небернуллиевские автоморфизмы (соответственно нестандартные последовательности) на грубые классы в зависимости от того, какова $\varepsilon$-энтропия в той или иной метрике множества случайных условных мер.

Стоит отметить, что свойства нестационарных убьвающих последовательностей, вообше говоря, сильно отличаются от свойств последовательностей прошлых (т.е. стационарных) и имеют совсем другие применения. Результаты и инварианты в теории убывающих последовательностей, например, введенная вьше энтропия, в равной мере относятся как к стационарному, так и к нестационарному случаю. Более существенно различие между однородными и неоднородными последовательностями. Основной интерес представляют однородные последовательности, например, диадические или неатомические (см. [39]). Главное их применение -в теории аппроксимации действий групп.

\section{СПИСОК ЛИТЕРАТУРЫ}

[1] Avez A. Entropie des groupes de type fini // C. R. Acad. Sci. Paris. 1972. V. 275. P. 1363-1366.

[2] Garsia A. Entropy and singularity of infinite convolutions // Pacific J. Math. 1963. V. 13. P. 1159-1169.

[3] Furstenberg H. A Poisson formula for semisimple Lie groups // Ann. of Math. (2). 1963. V. 77. P. 335-386.

[4] Furstenberg H. Random walks and discrete subgroups of Lie groups // Adv. Probab. Related Topics. 1971. V. 1. P. 3-63.

[5] Furstenberg H. Disjointness in ergodic theory, minimal sets and a problem in Diophantine approximation // Math. Systems Theory. 1967. V. 1. P. 1-49.

[6] Вершик А. М. Случайные блуждания на группах и близкие вопросы // Теория вероятн. и ее примен. 1981. Т. 26. №1. С. 190-191.

[7] Вершик А.М., Кайманович В.А. Случайные блуждания на группах: границы, энтропия, равномерные распределения // Докл. АН СССР. 1979. Т. 249. №1. С. 15-18.

[8] Kaimanovich V., Vershik A. Random walk on discrete groups: boundary and entropy // Ann. Probab. 1983. V. 11. № 3. P. 457-490.

[9] Kaimanovich V. Measure theoretic boundary of Markov chains, 0-2 law and entropy // Harmonic Analysis and Discrete Potential Theory / ed. M. Picardello. New York, 1990. P. $145-180$.

[10] Woess W. Random walks on infinite graphs and groups // Cambridge Tracts. 2000. V. 138 (to appear).

[11] Deriennic Y. Entropie, théorèmes limites et marches aléatoires // Lecture Notes in Math. 1986. V. 1210. P. 241-284.

[12] Gerl P. Random walks on graphs // Lecture Notes in Math. 1986. V. 1210. P. 285-303.

[13] Willis G. Probability measures on groups and some related ideals in group algebras // J. Funct. Anal. 1991. V. 92. P. 202-263.

[14] Ledrappier F. Une relation entre entropie, dimension et exposant pour certaines marches aléatoires // C. R. Acad. Sci. Paris. 1983. V. 296. P. 369-372.

[15] Ledrappier F. Some asymptotic properties of random walks on free groups // Preprint, 1997.

[16] Kaimanovich V. The Poisson boundary of covering Markov operators // Israel J. Math. 1995. V. 89. P. $77-134$.

[17] Kaimanovich V. Boundaries of invariant Markov operators: the identification problem // Proceedings of the Warwick Symposium 1993-94 / ed. M. Pollicott and K. Schmidt. Cambridge: Cambridge Univ. Press, 1996. P. 127-196. 
[18] Kaimanovich V. The Poisson formula for groups with hyperbolic properties // Preprint. Rennes: Univ. Rennes-1, 1997.

[19] Vershik A., Nechaev S., Bikbov R. Statistical properties of braid groups in locally free approximation // Comm. Math. Phys. 2000. V. 212. № 2. P. 469-501.

[20] Milnor J. Note on curvature and fundamental group // J. Differential Geom. 1968. V. 2. P. 1-7.

[21] Kesten H. Symmetric random walk on groups // Trans. Amer. Math. Soc. 1959. V. 92. P. 336-354.

[22] Kaimanovich V. Hausdorff dimension of the harmonic measure // Ergodic Theory Dynam. Systems. 1998. V. 18. № 3. P. 631-660.

[23] Буфетов А. Эргодические теоремы для свободных полугрупп // Функц. анализ и его прил. 2000. Т. 34 (в печати).

[24] Дынкин Е. Б., Малютов М. Б. Случайное блуждание на группах с конечным числом образуюших // Докл. АН СССР. 1961. Т. 137. С. 1042-1045.

[25] Masur H. Random walk on Teichmuller space and mapping class groups // J. Anal. Math. 1995. V. 67. P. 117-164.

[26] Kaimanovich V., Masur H. The Poisson boundary of Teichmuller space // J. Funct. Anal. 1998. V. 156. № 2. P. 301-332.

[27] Series C. Martin boundary of random walks of Fuchsian groups // Israel J. Math. 1983. V. 44. № 3. P. 221-242.

[28] Вершик А. М. Многозначные отображения с инвариантной мерой (полиморфизмы) и марковские операторы // Зап. науч. сем. ЛОМИ. 1977. Т. 72. С. 26-61.

[29] Vershik A. Amenability and approximation of infinite groups // Selecta Math. Soviet. 1982. V. 2. № 4. P. 311-330.

[30] Bisch D., Haagerup U. Composition of subfactors: New examples of infinite depth subfactors // Ann. Sci. Ecole Norm. Sup. 1996. V. 29. № 3. P. 329-383.

[31] Bisch D. Entropy of groups and subfactors // J. Funct. Anal. 1992. V. 103. № 1. P. 190-208.

[32] Вершик А. М. Локальные стационарные алгебры // Труды сибирск. школы "Алгебра и анализ" (Кемерово). М.: ВИНИТИ, 1988. С. 3-22.

[33] Vershik A. Local algebras and new version of Young's orthogonal form // Banach Center Publ. 1990. V. 26. Part 2. P. 467-473.

[34] Grosberg A., Nechaev S., Vershik A. Random walk on braid groups, Brownian motion, complexity and statistics // J. Phys. A. 1996. V. 29. P. 2411-2433.

[35] Desbois J., Nechaev S. Statistics of reduced words in locally free and braid groups // J. Statist. Phys. 1997. V. 88. P. 2767-2789.

[36] Cartier P., Foata D. Problèmes combinatoires de commutation et réarrangements. Berlin: Springer-Verlag, 1969. (Lecture Notes in Math. V. 85.)

[37] Viennot G.X. Heaps of pieces I. Basic definitions and combinatorial lemmas // Lecture Notes in Math. 1986. V. 1234. P. 321-350.

[38] Viennot G.X. Problèmes combinatoires posés par la physique statistique // Sém. Bourbaki. 1983/1984. Exp. 626; Astérisque. 1985. V. 121/122. P. 225-246.

[39] Вершик А. М. Убывающие последовательности измеримых разбиений // Алгебра и анализ. 1994. Т. 6. № 4. С. 1-68.

[40] Shields P., Ornstein D. Uncountably many of $K$-automorphisms // Adv. Math. 1973. V. 10. № 1. P. 63-88.

[41] Marton K., Shields P. How many future measures can there be? // Preprint. Hungary Academy, 1999.

[42] Вершик А. М. Численные характеристики групп и соотношения между ними // Зап. науч. сем. ПОМИ. 1999. Т. 240. С. 1-11.

[43] Gromov M. Hyperbolic groups // Math. Sci. Res. Inst. Publ. 1987. V. 8. P. 75-263.

[44] Гринлиф Ф. Инвариантные средние на группах. М.: Мир, 1973.

[45] Gromov M. Groups of polynomial growth and expanding maps // Inst. Hautes Études Sci. Publ. Math. 1981. V. 53. P. 53-78. 
[46] Григорчук Р. И. К проблеме Милнора роста в группах // Докл. АН СССР. 1983. T. 271. №3. C. $30-33$.

[47] Epstein D., Cannon J., Holt D., Levy S., Paterson M., Thurston W. Word Processing in Groups. Boston: Jones and Bartlett Publ., 1992.

[48] Grigorchuk R., Nagnibeda T. Complete growth functions of hyperbolic groups // Invent. Math. 1997. V. 130. P. 159-188.

[49] de la Harpe P. Topics in Geometric Groups Theory. Chicago: Univ. Chicago Press (to appear).

[50] de la Harpe P., Grigorchuk R. On problems related to growth, entropy, and spectrum in group theory // J. Dynam. Control Systems. 1997. V. 3. № 1. P. 51-89.

[51] Ревюз Д. Цепи Маркова. М.: РФФИ, 1996.

[52] Varopoulos N., Saloff-Coste L., Coulhon T. Analysis on Lie Groups. Cambridge: Cambridge Univ. Press, 1992.

[53] Varopoulos N. Long range estimates for Markov chains // Bull. Sci. Math. 1985. V. 109. P. $225-252$.

[54] Спитцер Ф. Принципы случайных блужданий. М.: Мир, 1968.

[55] Дюбина А. Г. Пример скорости ухода на бесконечность случайного блуждания на группах // УМН. 1999. Т. 54. № 5. С. 159-160.

[56] Rosenblatt M. Markov Processes, Structure and Asymptotic Behavior. Berlin: SpringerVerlag, 1971.

[57] Вершик А. М. Сверхгрубость гиперболических автоморфизмов и унитарные дилатации марковских операторов // Вестн. ЛГУ. Сер. матем. 1987. № 3. С. 28-33.

[58] Динабург Е. И. Соотношения между топологической энтропией и метрической энтропией // Докл. АН СССР. 1970. Т. 190. № 1. С. 19-22.

[59] Вершик А. М. Описание инвариантных мер для действий некоторых бесконечномерных групп // Докл. АН СССР. 1974. Т. 218. № 4. С. 749-752.

[60] Кайманович В. А. Броуновское движение и гармонические функции на накрывающих многообразиях. Энтропийный подход // Докл. АН СССР. 1986. Т. 288. № 5. С. 1045-1049.

[61] Марков А. А., Нагорный Н. М. Теория алгорифмов. М.: Фазис, 1998.

[62] Адян С.И. Об алгоритмическом распознавании свойств групп // Докл. АН СССР. 1957. T. 117. № 4. C. 9-12.

[63] Ghys E., de la Harpe P. Infinite groups as geometric objects (after Gromov) // Ergodic Theory, Symbolic Dynamics, and Hyperbolic Spaces. Lect. Workshop Hyperbolic Geom. Ergodic Theory (Trieste, 1989). New York, 1991. P. 299-314.

[64] Zimmer R. Ergodic Theory and Semisimple Groups. Boston: Birkhäuser, 1984.

[65] Connes A., Feldman J., Weiss B. An amenable equivalence relation is generated by a single transformation // Ergodic Theory Dynam. Systems. 1981. V. 1. P. 431-450.

[66] Дынкин Е. Б. Пространство выходов марковских процессов // УМН. 1969. Т. 24. № 3. С. $89-152$.

[67] Krengel U. Ergodic Theorems. Berlin: de Gruyter, 1985.

[68] Рохлин В. А.Лекции по энтропийной теории преобразований с инвариантной мерой // УМH. 1967. Т. 22. №5. С. 4-56.

[69] Вершик А. М. Убьвающие последовательности измеримых разбиений и их применения // Докл. АН СССР. 1970. Т. 193. С. 748-751.

[70] Bowen R. Topological entropy for noncompact sets // Trans. Amer. Math. Soc. 1973. V. 184. P. $125-136$.

[71] Jewett R. The prevalence of uniquely ergodic systems // J. Math. Mech. 1970. V. 19. P. $717-729$

[72] Krieger W. On unique ergodicity // Proc. Sixth Berkeley Symp. V. 1, 1970. P. 327-346.

[73] Гуревич Б. М., Савченко С. В. Термодинамический формализм для символических цепей Маркова со счетным числом состояний // УМН. 1998. Т. 53. № 2. С. 3-106.

[74] Холл М. Теория групп. М., 1962.

[75] Magnus W. On a theorem of M. Hall // Ann. of Math. 1939. V. 40. P. 764-768. 
[76] Шмелькин А. Л. Сплетения и многообразия групп // Изв. АН СССР. Сер. матем. 1965. Т. 29. № 1. С. 149-176.

[77] Соколов В. Г. Аналог формулы Витта для свободных разрешимых групп // Алгебра и логика. 1969. Т. 8. №3. С. 367-372.

[78] Петроградский В. М. О некоторых типах промежуточного роста в алгебрах Ли // УМH. 1993. Т. 48. № 5. С. 181-182.

[79] Lyons R., Pemantle R., Peres Y. Random walk on the lamplighter group // Ann. Probab. 1996. V. 24. № 4. P. 1993-2006.

[80] Вершик А.М., Граничина О.А. Редукция неголономных вариационных задач к изопериметрическим и связности в главных расслоениях // Матем. заметки. 1991. Т. 49. №5. C. $37-44$.

[81] Collins R. Relation among the squares of the generators of the braid groups // Invent. Math. 1994. V. 117. №3. P. 525-529.

[82] Humphries S. On representations of Artin Groups and the Tits Conjecture // J. Algebra. 1994. V. 169. P. 847-862.

[83] Okounkov A., Vershik A. New approach to representation theory of symmetric groups // Selecta Math. 1996. V. 2. № 4. P. 581-605.

[84] Derrida B., Leibovitz J. Exact large deviation function in the asymmetric exclusion process // Phys. Rev. A. 1986. V. 34. P. 5091-5095.

[85] Bikbov R., Nechaev S. On the limiting power of the set of knots generated by $1+1$ and $2+1$ braids // J. Math. Phys. 1999. V. 40. № 12. P. 6598-6608.

[86] Comtet A., Nechaev S. Random operator approach for word enumeration in braid groups // J. Phys. A. 1988. V. 31. P. 5609-5630.

[87] Малышев В. А. Случайные грамматики // УМН. 1998. Т. 53. № 2. С. 107-134.

[88] Колмогоров А. Н. Новый метрический инвариант транзитивных динамических систем и автоморфизмов пространств Лебега // Докл. АН СССР. 1958. Т. 119. № 5. С. 861-864.

[89] Колмогоров А.Н. Об энтропии на единицу времени как метрическом инварианте автоморфизмов // Докл. АН СССР. 1959. Т. 124. С. 759-765.

[90] Рохлин В.А.Новьй прогресс в теории преобразований с инвариантной мерой // УМН. 1960. Т. 15. № 4. С. 3-28.

[91] Синай Я.Г. О понятии энтропии динамической системы // Докл. АН СССР. 1959. T. 124. № 4. C. $768-771$.

[92] Sinai Ya. Probabilistic ideas in ergodic theory // Proc. Int. Congr. Math. Stockholm, 1962.

[93] Корнфельд И.П., Синай А.Г., Фомин С. В. Эргодическая теория. М.: Наука, 1975.

[94] Синай А. Г. (ред.) Динамические системы-2 // Итоги науки и техники. Совр. пробл. матем. Фунд. напр. М.: ВИНИТИ, 1985.

[95] Биллингсли П. Эргодическая теория и информация. М.: Мир, 1970.

[96] Мартин Н., Ингланд Дж. Математическая теория энтропии. М.: Мир, 1989.

[97] Smorodinsky M. Ergodic Theory, Entropy. Berlin: Springer-Verlag, 1971. (Lecture Notes in Math. V. 214.)

[98] Ornstein D., Weiss B. Entropy theory and isomorphism theorems for action of amenable groups // J. Anal. Math. 1987. V. 48. P. 1-141.

[99] Вершик А. М. Континуум попарно неизоморфных диадических последовательностей // Функц. анализ и его прил. 1971. Т. 5. № 3. С. 16-18.

[100] Степин А. М. Об энтропийном инварианте убывающих последовательностей измеримых разбиений // Функц. анализ и его прил. 1971. Т. 5. № 2. С. 80-84.

[101] Канторович Л. В. О перевозке масс // Докл. АН СССР. 1942. Т. 37. С. 227-229.

[102] Канторович Л. В., Рубинштейн Г. Ш. О пространстве вполне аддитивных функций // Вестн. ЛГУ. 1958. № 2. С. 52-59.

[103] Dubins L., Feldman J., Smorodinsky M., Tsirel'son B. Decreasing sequences of $\sigma$-fields and a measure change for Brownian motion // Ann. Probab. 1996. V. 24. P. 882-904. 
[104] Heicklen D., Hoffman C. $\left(T, T^{-1}\right)$ is not standard // Ergodic Theory Dynam. Systems. 1998. V. 18. P. 875-879.

[105] Орнстейн Д. Эргодическая теория, случайность и динамические системы. М.: Мир, 1978.

[106] Kalikow S. (T, $\left.T^{-1}\right)$-transformation is not loosely Bernoulli // Ann. of Math. (2). 1982. V. 115. P. 393-409.

[107] Feldman J. New $K$-automorphism and a problem of Kakutani // Israel J. Math. 1976. V. 24. P. $16-38$.

[108] Каток А.Б. Монотонная эквивалентность в эргодической теории // Изв. АН СССР. 1977. T. 41. № 1. C. 104-157.

С.-Петербургское отделение

Поступила в редакцию

Математического института им. В. А. Стеклова

09.03.2000 